\title{
The highs and lows of programmed cardiovascular disease by developmental hypoxia
}

Citation for published version (APA):

Salinas-Salmón, C. E. (2017). The highs and lows of programmed cardiovascular disease by developmental hypoxia: studies in the chicken embryo. [Doctoral Thesis, Maastricht University]. Maastricht University. https://doi.org/10.26481/dis.20171213cs

Document status and date:

Published: 01/01/2017

DOI:

10.26481/dis.20171213cs

Document Version:

Publisher's PDF, also known as Version of record

\section{Please check the document version of this publication:}

- A submitted manuscript is the version of the article upon submission and before peer-review. There can be important differences between the submitted version and the official published version of record.

People interested in the research are advised to contact the author for the final version of the publication, or visit the DOI to the publisher's website.

- The final author version and the galley proof are versions of the publication after peer review.

- The final published version features the final layout of the paper including the volume, issue and page numbers.

Link to publication

\footnotetext{
General rights rights.

- You may freely distribute the URL identifying the publication in the public portal. please follow below link for the End User Agreement:

www.umlib.nl/taverne-license

Take down policy

If you believe that this document breaches copyright please contact us at:

repository@maastrichtuniversity.nl

providing details and we will investigate your claim.
}

Copyright and moral rights for the publications made accessible in the public portal are retained by the authors and/or other copyright owners and it is a condition of accessing publications that users recognise and abide by the legal requirements associated with these

- Users may download and print one copy of any publication from the public portal for the purpose of private study or research.

- You may not further distribute the material or use it for any profit-making activity or commercial gain

If the publication is distributed under the terms of Article $25 \mathrm{fa}$ of the Dutch Copyright Act, indicated by the "Taverne" license above, 


$$
\text { siney }
$$


Cover design: Javier Sánchez-Villamor

ISBN/EAN:978-94-028-0874-2 


\title{
THE HIGHS AND LOWS OF PROGRAMMED CARDIOVASCULAR DISEASE BY DEVELOPMENTAL HYPOXIA: STUDIES IN THE CHICKEN EMBRYO
}

\author{
Dissertation \\ to obtain the degree of Doctor at the Maastricht University, \\ on the authority of the Rector Magnificus, \\ Prof. dr. Rianne M. Letschert \\ in accordance with the decision of the Board of Deans, \\ to be defended in public \\ on 13 of December 2017, at 11:30 hours
}

by

Carlos Eduardo Salinas-Salmón 


\section{Supervisors:}

Prof. dr. Luc J.I. Zimmermann

Prof. dr. Dino Giussani (University of Cambridge)

Prof. dr. Carlos E.Blanco (Royal College of Surgeons of Ireland)

\section{Co-supervisor:}

Dr. Eduardo Villamor

\section{Assessment committee:}

- Prof. dr. Boris W. Kramer (Chairman)

- Prof. dr. Frits W. Prinzen

- Prof. dr. Wim van Gemert

- Prof. dr. Máximo Vento (Universidad de Valencia)

- Prof. dr. Francisco Pérez-Vizcaino (Universidad Complutense de Madrid) 


\section{Contents}

Chapter I Introduction, aims, and outline of the thesis

Chapter II The role of oxygen in prenatal growth: studies in the chick embryo. $J$ Physiol. 2007; 585:911-7

Chapter III Cardiac and vascular disease prior to hatching in chick embryos incubated at high altitude. $J$ Dev Orig Health Dis. 2010; 1:60-6

Chapter IV Adrenocortical suppression in highland chick embryos is restored during incubation at sea level. High Alt Med Biol. 2011; 12:79-87

Chapter V High altitude hypoxia and blood pressure dysregulation in adult chickens. $J$ Dev Orig Health Dis. 2013; 4:69-76

Chapter VI High-altitude hypoxia and echocardiographic indices of pulmonary hypertension in male and female chickens at adulthood. Circ J. 2014;78:1459-64

Chapter VII General discussion

Adapted from The highs and lows of programmed cardiovascular disease by developmental hypoxia: Studies in the chicken embryo. J Physiol 2017 [Epub ahead of print] 


$$
=
$$





\section{CHAPTER I: INTRODUCTION, AIMS AND OUTLINE OF THE THESIS}

\section{Developmental origins of cardiovascular health and disease}

Cardiovascular disease is the leading cause of death in the world and is associated with high morbidity and cost $(1,2)$. It is widely accepted that the risk of developing the most common cardiovascular and metabolic diseases in our society - hypertension, diabetes, dyslipidaemia and coronary heart disease - is determined by the interaction between our genetic make-up and traditional lifestyle risk factors, such as smoking, obesity and/or a sedentary life. Nevertheless, we now understand that adverse environmental conditions during early life, such as pre-conceptually and during pregnancy, may be just as if not more important than postnatal lifestyle in interacting with genes to set a risk of cardiovascular disease $(3,4)$.

The Barker hypothesis states that low birth weight is associated with an increased risk of cardiovascular disease and its associated disorders (stroke, type 2 diabetes, hypertension and metabolic syndrome) in adulthood. In this scenario, low birth weight is a surrogate measure for adverse intrauterine conditions in complicated pregnancy. The first epidemiological studies, which support the Barker hypothesis came from Forsdahl (5). This study reported that the same regions in Norway shared an increased prevalence of complications during infancy, such as low birth weight and poor prognosis during infancy and an increased incidence of cardiovascular disease in adulthood (5). Later, Barker and Osmond reported that the prevalence of cardiovascular mortality coincided with greater neonatal mortality in the same areas of England and Wales during the first decades of the 20 th century $(6,7)$. This finding triggered the idea that poor intrauterine growth, indexed by low birth weight, was associated with an increased risk of coronary heart disease in later life (8). Subsequently, fetal malnutrition was associated with an increased prevalence of insulin resistance (9), hypertension (10), hyperlipidaemia (11) and elevated plasma levels of fibrinogen in the offspring in later life (12). The idea that fetal malnutrition could have long term effects on the physiology of the offspring is not in fact new. The classical studies of Widdowson and McCance showed in animals that those with low weight at birth never reach the same size or maturity at adulthood compared with those born with normal birth weight $(13,14)$. It was postulated that a decrease in cellular endowment in key organs, such as in the heart and kidney imposed by adverse intrauterine conditions, might underlie the relationship $(13,14)$. 


\section{Normal and slow fetal growth}

The Barker hypothesis has now been expanded into the concept of Developmental Origins of Health and Disease (DOHAD)(3,4). Since intrauterine growth restriction (IUGR) is an established surrogate marker for adverse intrauterine conditions, there has been a keen scientific and clinical interest in identifying which and how sub-optimal environmental conditions may promote IUGR and programme an increased cardiovascular risk in the offspring. Fetal growth and development is believed to be determined by three main factors: the nutritional state of the pregnant mother, placental function and the fetal capacity to utilise substrates for energy production.

-Maternal nutritional status during pregnancy. It is known that low maternal food intake and/or impaired nutrient absorption can cause slow fetal growth and IUGR (15). Nevertheless, the individual variability in the response to protein and energy constraint is vast. It is now known that undernutrition during pregnancy can induce permanent effects in the offspring, such as a reduction in tissue cell number, organ structural remodelling, changes in key hormone levels as well as epigenetic changes. The specific long-term effects of maternal malnutrition will clearly depend on the duration and magnitude of the challenge. Every organ has its own critical and sensitive period of cellular replication during which it will be most affected by adverse developmental conditions (16). For instance, both hypo- and hyperglycaemia during early embryogenesis have been associated with low birth weight. While maternal undernutrition during early gestation triggers compensatory placental growth that protects fetal growth, maternal undernutrition during late gestation significantly impairs fetal growth and triggers cardiometabolic sequelae in the offspring. The latter is illustrated by the observations of the "Dutch Hunger Winter", which was a famine that took place in the German-occupied part of the Netherlands, especially in the densely populated western provinces north of the great rivers, during the winter of 1944-45, near the end of World War II. It was reported that children born to mothers with significantly reduced food intake $(400-800 \mathrm{kcal} /$ day) during the last third of pregnancy were of low birth weight and developed glucose intolerance and insulin resistance at adulthood (17). This relationship between maternal malnutrition and glucose intolerance in the offspring in later life could be explained by a permanent effect of the challenge on fetal pancreatic beta cell endowment, with long term consequences for changes in insulin sensitivity in target organs later in life (17).

- Placental function. It is well established that placental insufficiency causes a slowing of fetal growth. The lack or inappropriate development of the placental vascular bed will 
increase placental vascular resistance which will reduce delivery of oxygen and nutrients to the growing conceptus. In addition, infarcts and oxidative stress will further impact on functional placental tissue, aggravating the reduction in the appropriate supply of nutrients to the fetus $(18,19)$. It is also accepted that there may be placental adaptation to adverse or stressful conditions during pregnancy that may cushion the impact of adversity on fetal growth and development. For instance, anaemia during pregnancy (20), maternal exercise (21) and high altitude pregnancy (22) have all been reported to trigger an increase in placental mass with partial protection on fetal growth. Studies in animal models have also reported that pre-conceptual overnutrition rather than an increase in food intake during early pregnancy, leads to an increase in placental growth with benefits to fetal growth (23). Therefore, it is clear that impaired placental function will adversely affect fetal growth and that the placenta can adapt beneficially to ameliorate the effects of adverse pregnancy on fetal growth, depending on the timing, duration and magnitude of the suboptimal condition.

-Fetal capacity to utilize energy substrate: It must also be accepted that despite appropriate maternal nutrition and adequate placental development and function, there are circumstances in which fetal growth may still be compromised. This is the case in chromosomal abnormalities, uterine or fetal malformations or during intrauterine infection (24).

\section{Chronic fetal hypoxia, fetal growth and cardiovascular risk in later life}

In addition to alterations in maternal nutrition and placental function, significant data derived from human epidemiological studies and from experimental animal models of pregnancy resulting in IUGR now clarified that a component of the slowed fetal growth and the setting of an increased cardiovascular risk in later life may be due specifically to longterm reductions in fetal oxygenation or chronic fetal hypoxia. Nevertheless, the contribution of chronic fetal hypoxia in promoting IUGR and programmed cardiovascular risk has been difficult to isolate for a number of reasons. For instance, it is established that high-altitude pregnancy leads to IUGR (25-28). However, most high-altitude populations are impoverished with significant maternal malnutrition $(26,29,30)$. Therefore, the contribution of chronic fetal hypoxia versus chronic fetal undernutrition in slowing fetal growth and in setting future cardiovascular risk under these conditions is uncertain. The same applies to sea level pregnancy complicated by preeclampsia, placental insufficiency, gestational diabetes and even maternal obesity. All these conditions are associated with an increase in placental vascular resistance and alterations in placental development and 
function (31-35), which will decrease oxygen as well as nutrient delivery to the growing fetus. Similarly, several studies in mammalian experimental animal models have shown that maternal chronic hypoxia during pregnancy can lead to IUGR and programme increased cardiovascular risk in the offspring (36-40). However, because experimental induction of chronic hypoxia in rodents can reduce maternal food intake and/or alter the quality of the maternal milk $(36,41-43)$, the contribution of chronic fetal hypoxia versus chronic fetal and/or neonatal under-nutrition under these conditions, again, remains uncertain.

\section{The chicken embryo model}

By using the chicken embryo as an animal model, science has been able to circumvent a number of these problems highlighted above because, in contrast to mammals, with the exception of monotremes, in the chicken the effects of changes in oxygenation on the embryo can be isolated and determined directly, independent of changes in the maternal physiology, the secretion of placental hormones or imposed changes in postnatal maternal lactation (44-47).

The development of the chicken embryo during the 21 days of incubation is supported by three gas exchange organ systems: the yolk sac, the chorioallantoic membrane (CAM) and the lungs $(48,49)$. The vascular portion of the yolk sac is the principal gas exchange organ during early development (50). In addition to its role as an early gas exchange organ, the yolk sac provides the chicken embryo with essential nutrients for its growth. Protein, fat, carbohydrates and minerals are stored in the yolk of the egg for future utilization by the embryo $(51,52)$. At approximately embryonic day 8 , the allantoic sac develops and fuses with the chorion to create the CAM (49). This highly vascular structure, in conjunction with the porosity of the eggshell, permits diffusion of oxygen and carbon dioxide between the environment and the blood, thereby replacing the yolk sac as the primary source of oxygen uptake $(48,49,53)$. Finally, transition to ex ovo life in chickens is initiated around incubation day 19-20, when the embryo 'pips' internally through to the air cell of the egg with its beak and begins air breathing for the first time (54). From this point on, the embryo relies on both the CAM and the lungs for gas exchange. Therefore, during the second half of incubation, chicken embryos are endowed with a gas-exchange organ, the CAM, and a choriovitelline nutritional organ, the yolk sac $(48,49,53,54)$. Hypoxia is easily induced by incubating the egg in a low $\mathrm{O}_{2}$ environment. Exposure to hypoxia during midincubation increased CAM vascularity, resulting in an increased functional surface area for gas exchange, which would be an appropriate acclimation to hypoxia (55-57). 


\section{AIMS AND OUTLINE OF THE THESIS}

The present thesis is a collection of studies designed to isolate the effects of chronic fetal hypoxia on fetal growth, fetal cardiovascular and endocrine development and programming of future cardiovascular dysfunction in the adult offspring, using the chicken as an animal model. The combination of high altitude exposure with the use of the chicken embryo model is ideal as it permits investigation of the direct effects of high altitude hypoxia on growth and on cardiovascular development completely independent of alterations in placental function, independent of changes in the maternal physiology and independent of any effects of socioeconomic factors.

In chapter II (The role of oxygen in prenatal growth: studies in the chick embryo. J Physiol. 2007; 585:911-7), chapter III (Cardiac and vascular disease prior to hatching in chick embryos incubated at high altitude. J Dev Orig Health Dis. 2010; 1:60-6), and chapter IV (Adrenocortical suppression in highland chick embryos is restored during incubation at sea level. High Alt Med Biol. 2011; 12:79-87), we investigated the effects of high altitude hypoxia on chicken embryo growth and in ovo cardiovascular and endocrine development. For this purpose, we adopted an experimental design based on a three-prong approach using: (1) incubation at high altitude of fertilized eggs laid by sea-level hens; (2) incubation at sea level of fertilized eggs laid by high-altitude hens; and (3) incubation at high altitude of sea-level eggs with oxygen supplementation to equate sea level oxygen partial pressure.

In chapter $\mathbf{V}$ (High altitude hypoxia and blood pressure dysregulation in adult chickens. $\mathrm{J}$ Dev Orig Health Dis. 2013; 4:69-76) and chapter VI (High-altitude hypoxia and echocardiographic indices of pulmonary hypertension in male and female chickens at adulthood. Circ J. 2014;78:1459-64), we isolated the long-term consequences of chronic hypoxic incubation of chick embryos on the systemic and pulmonary circulations of the adult bird. This was achieved using noninvasive echocardiography as well as testing basal and stimulated cardiovascular function in the chronically instrumented adult bird. Additional specific points of interest were to determine whether there were any sex differences and whether any adverse effects of chronic hypoxia during the embryonic period could be ameliorated by generational exposure to hypobaric hypoxia in highland adapted chickens.

Finally, in chapter VII (The highs and lows of programmed cardiovascular disease by developmental hypoxia: Studies in the chicken embryo. J Physiol 2017), we discuss and 
put into perspective the findings of this thesis. We summarise studies that have exploited the chicken embryo model to isolate the direct effects of chronic hypoxia on prenatal growth, cardiovascular and endocrine development and in triggering an increased risk of cardiovascular dysfunction and pathology at adulthood.

\section{References}

1. Reddy KS, Yusuf S 1998 Emerging epidemic of cardiovascular disease in developing countries. Circulation 97:596-601.

2. Yusuf S, Reddy S, Ônpuu S, Anand S 2001 Global burden of cardiovascular diseases. Circulation 104:2855-2864.

3. Gluckman PD, Hanson MA, Cooper C, Thornburg KL 2008 Effect of in utero and early-life conditions on adult health and disease. N Engl J Med 359:61-73.

4. Barker DJ, Gluckman PD, Godfrey KM, Harding JE, Owens JA, Robinson JS 1993 Fetal nutrition and cardiovascular disease in adult life. Lancet 341:938941.

5. Forsdahl A 1977 Are poor living conditions in childhood and adolescence an important risk factor for arteriosclerotic heart disease? Journal of Epidemiology \& Community Health 31:91-95.

6. Barker DJ, Osmond C 1986 Infant mortality, childhood nutrition, and ischaemic heart disease in England and Wales. Lancet 327:1077-1081.

7. Barker D, Osmond C 1987 Death rates from stroke in England and Wales predicted from past maternal mortality. Br Med J (Clin Res Ed) 295:83-86.

8. Barker DJ, Godfrey KM, Gluckman PD, Harding JE, Owens JA, Robinson JS 1993 Fetal nutrition and cardiovascular disease in adult life. Lancet 341:938941.

9. Hales C, Barker D, Clark P, Cox L, Fall C, Osmond C, Winter P 1991 Fetal and infant growth and impaired glucose tolerance at age 64. Bmj 303:1019-1022.

10. Law CM, Shiell AW 1996 Is blood pressure inversely related to birth weight? The strength of evidence from a systematic review of the literature. J Hypertens 14:935-942.

11. Barker D, Martyn C, Osmond C, Hales C, Fall C 1993 Growth in utero and serum cholesterol concentrations in adult life. Bmj 307:1524-1527.

12. Martyn C, Meade T, Stirling Y, Barker D 1995 Plasma concentrations of fibrinogen and factor VII in adult life and their relation to intra-uterine growth. Br J Haematol 89:142-146.

13. Widdowson E, McCance R 1974 The determinants of growth and form. Proc R Soc Lond, pp 1-17.

14. Widdowson E, McCance R 1975 A review: new thoughts on growth. Pediatr Res 9:154-156.

15. Ceesay SM, Prentice AM, Cole TJ, Foord F, Poskitt EM, Weaver LT, Whitehead RG 1997 Effects on birth weight and perinatal mortality of maternal dietary supplements in rural Gambia: 5 year randomised controlled trial. Bmj 315:786-790.

16. Becerra Fernández A 1999 Malnutrición fetal y enfermedad metabólica en la vida adulta. Nutrición y Obesidad 251.

17. Ravelli AC, van der Meulen JH, Michels R, Osmond C, Barker DJ, Hales C, Bleker OP 1998 Glucose tolerance in adults after prenatal exposure to famine. Lancet 351:173-177.

18. Lin C-C 2012 Fetal growth retardation. In Lin C-C, Verp M, Sabbagha RE (eds) The high-risk fetus: pathophysiology, diagnosis, and management. Springer Science \& Business Media.

19. Kingdom J, Kaufmann P 1997 Oxygen and placental villous development: origins of fetal hypoxia. Placenta 18:613-621. 
20. Godfrey K, Redman C, Barker D, Osmond C 1991 The effect of maternal anaemia and iron deficiency on the ratio of fetal weight to placental weight. BJOG: An International Journal of Obstetrics \& Gynaecology 98:886-891.

21. Clapp JF, Rizk KH 1992 Effect of recreational exercise on midtrimester placental growth. Am J Obstet Gynecol 167:1518-1521.

22. Mayhew T, Jackson MR, Haas J 1990 Oxygen diffusive conductances of human placentae from term pregnancies at low and high altitudes. Placenta 11:493503.

23. Goldberg GR, Prentice AM 1994 Maternal and fetal determinants of adult diseases. Nutr Rev 52:191-200.

24. Sagi-Dain L, Peleg A, Sagi S 2017 Risk for chromosomal aberrations in apparently isolated intra-uterine growth restriction-a systematic review. Prenatal Diagnosis.

25. Moore LG 1990 Maternal O-2 Transport and Fetal Growth in Colorado, Peru, and Tibet High-Altitude Residents. Am J Hum Biol 2:627-637.

26. Giussani DA, Phillips PS, Anstee S, Barker DJ 2001 Effects of altitude versus economic status on birth weight and body shape at birth. Pediatr Res 49:490494.

27. Moore LG, Charles SM, Julian CG 2011 Humans at high altitude: hypoxia and fetal growth. Respir Physiol Neurobiol 178:181-190.

28. Soria R, Julian CG, Vargas E, Moore LG, Giussani DA 2013 Graduated effects of high-altitude hypoxia and highland ancestry on birth size. Pediatr Res 74:633-638.

29. Niermeyer S, Zamudio S, Moore LG 2001 The People. In: High altitude : an exploration of human adaptation. Marcel Dekker, New York, pp xx, 982 p.

30. Julian CG, Wilson MJ, Moore LG 2009 Evolutionary adaptation to high altitude: a view from in utero. Am J Hum Biol 21:614-622.

31. Okamura K, Watanabe T, Tanigawara S, Shintaku Y, Endo H, Iwamoto M, Murotsuki J, Yajima A 1990 Biochemical evaluation of fetus with hypoxia caused by severe preeclampsia using cordocentesis. J Perinat Med 18:441-447.

32. Gagnon R 2003 Placental insufficiency and its consequences. Eur J Obstet Gynecol Reprod Biol 110 Suppl 1:S99-107.

33. Kendall G, Peebles D 2005 Acute fetal hypoxia: the modulating effect of infection. Early Hum Dev 81:27-34.

34. Macfarlane CM, Tsakalakos N 1985 Evidence of hyperinsulinaemia and hypoxaemia in the cord blood of neonates born to mothers with gestational diabetes. S Afr Med J 67:81-84.

35. Hayes EK, Lechowicz A, Petrik JJ, Storozhuk Y, Paez-Parent S, Dai Q, Samjoo IA, Mansell M, Gruslin A, Holloway AC, Raha S 2012 Adverse fetal and neonatal outcomes associated with a life-long high fat diet: role of altered development of the placental vasculature. PLoS One 7:e33370.

36. Camm EJ, Hansell JA, Kane AD, Herrera EA, Lewis C, Wong S, Morrell NW, Giussani DA 2010 Partial contributions of developmental hypoxia and undernutrition to prenatal alterations in somatic growth and cardiovascular structure and function. Am J Obstet Gynecol 203:495 e424-434.

37. Giussani DA, Camm EJ, Niu Y, Richter HG, Blanco CE, Gottschalk R, Blake EZ, Horder KA, Thakor AS, Hansell JA, Kane AD, Wooding FB, Cross CM, Herrera EA 2012 Developmental programming of cardiovascular dysfunction by prenatal hypoxia and oxidative stress. PLoS One 7:e31017.

38. Thompson JA, Richardson BS, Gagnon R, Regnault TR 2011 Chronic intrauterine hypoxia interferes with aortic development in the late gestation ovine fetus. J Physiol 589:3319-3332.

39. Thompson LP, Weiner CP 1999 Effects of acute and chronic hypoxia on nitric oxide-mediated relaxation of fetal guinea pig arteries. Am J Obstet Gynecol 181:105-111.

40. Giussani DA 2016 The fetal brain sparing response to hypoxia: physiological mechanisms. J Physiol 594:1215-1230. 
41. Williams SJ, Hemmings DG, Mitchell JM, McMillen IC, Davidge ST 2005 Effects of maternal hypoxia or nutrient restriction during pregnancy on endothelial function in adult male rat offspring. J Physiol 565:125-135.

42. O'Dowd R, Kent JC, Moseley JM, Wlodek ME 2008 Effects of uteroplacental insufficiency and reducing litter size on maternal mammary function and postnatal offspring growth. Am J Physiol Regul Integr Comp Physiol 294:R539548.

43. Wlodek ME, Westcott KT, O'Dowd R, Serruto A, Wassef L, Moritz KM, Moseley JM 2005 Uteroplacental restriction in the rat impairs fetal growth in association with alterations in placental growth factors including PTHrP. Am J Physiol Regul Integr Comp Physiol 288:R1620-1627.

44. Ruijtenbeek K, De Mey JG, Blanco CE 2002 The chicken embryo in developmental physiology of the cardiovascular system: a traditional model with new possibilities. Am J Physiol Regul Integr Comp Physiol 283:R549-550; author reply R550-541.

45. Mulder AL, Miedema A, De Mey JG, Giussani DA, Blanco CE 2002 Sympathetic control of the cardiovascular response to acute hypoxemia in the chick embryo. Am J Physiol Regul Integr Comp Physiol 282:R1156-1163.

46. Mulder AL, van Goor CA, Giussani DA, Blanco CE 2001 Alpha-adrenergic contribution to the cardiovascular response to acute hypoxemia in the chick embryo. Am J Physiol Regul Integr Comp Physiol 281:R2004-2010.

47. Mulder AL, Golde JM, Goor AA, Giussani DA, Blanco CE 2000 Developmental changes in plasma catecholamine concentrations during normoxia and acute hypoxia in the chick embryo. J Physiol 527 Pt 3:593-599.

48. Druyan S, Cahaner A, Ashwell C 2007 The expression patterns of hypoxiainducing factor subunit a-1, heme oxygenase, hypoxia upregulated protein 1 , and cardiac troponin $\mathrm{T}$ during development of the chicken heart. Poultry science 86:2384-2389.

49. Wangensteen OD, Rahn H 1971 Respiratory gas exchange by the avian embryo. Respiration Physiology 11:31-45.

50. Baumann R, Meuer H-J 1992 Blood oxygen transport in the early avian embryo. Physiological reviews 72:941-965.

51. Yadgary L, Kedar O, Adepeju O, Uni Z 2013 Changes in yolk sac membrane absorptive area and fat digestion during chick embryonic development. Poult Sci 92:1634-1640.

52. Speake BK, Murray AM, Noble RC 1998 Transport and transformations of yolk lipids during development of the avian embryo. Prog Lipid Res 37:1-32.

53. Mohammed R, Cavallaro G, Kessels CG, Villamor E 2015 Functional differences between the arteries perfusing gas exchange and nutritional membranes in the late chicken embryo. Journal of Comparative Physiology B 185:783-796.

54. Dzialowski EM, Sirsat T, van der Sterren S, Villamor E 2012 Prenatal cardiovascular shunts in amniotic vertebrates. Respir Physiol Neurobiol 178:66-74.

55. Chan T, Burggren W 2005 Hypoxic incubation creates differential morphological effects during specific developmental critical windows in the embryo of the chicken (Gallus gallus). Respiratory physiology \& neurobiology 145:251-263.

56. Strick DM, Waycaster RL, Montani J-P, Gay WJ, Adair TH 1991 Morphometric measurements of chorioallantoic membrane vascularity: effects of hypoxia and hyperoxia. American Journal of Physiology-Heart and Circulatory Physiology 260:H1385-H1389.

57. Verhoelst E, De Ketelaere B, Bruggeman V, Villamor E, Decuypere E, De Baerdemaeker J 2011 Development of a fast, objective, quantitative methodology to monitor angiogenesis in the chicken chorioallantoic membrane during development. International Journal of Developmental Biology 55:85-92. 
Chapter II

The role of oxygen in prenatal growth: studies in the chick embryo.

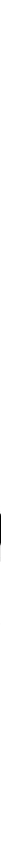

J Physiol. 2007; 585:911-7 



\title{
The role of oxygen in prenatal growth: studies in the chick embryo
}

\author{
Dino A. Giussani ${ }^{1}$, Carlos E. Salinas ${ }^{2}$, Mercedes Villena ${ }^{2}$ and Carlos E. Blanco ${ }^{3}$ \\ ${ }^{1}$ Department of Physiology Development and Neuroscience, University of Cambridge, Downing Street, Cambridge CB2 3EG, UK \\ ${ }^{2}$ Instituto Boliviano de Biología de Altura, Facultad de Medicina, Universidad Mayor de San Andrés, La Paz, Bolivia \\ ${ }^{3}$ Department of Pediatrics, Maastricht University, Maastricht, the Netherlands
}

The compelling evidence linking small size at birth with later cardiovascular disease has renewed and amplified scientific and clinical interests into the determinants of fetal growth. It is accepted that genes and nutrition control fetal growth; however, prior to this study, it had been impossible to isolate the effect of increases and decreases in fetal oxygenation on the regulation of prenatal growth. We investigated the role of oxygen in the control of fetal growth in the chicken because in contrast to mammals, the effects on the fetus of changes in oxygenation could be isolated, by assessing them directly without alteration to the maternal or placental physiology or maternal nutrition during development. The data show that incubation at high altitude of fertilized eggs laid by sea level hens markedly restricted fetal growth. Incubation at high altitude of fertilized eggs laid by high altitude hens also restricted fetal growth, but to a lesser extent compared to eggs laid by sea level hens. By contrast, incubation at sea level of fertilized eggs laid by high altitude hens not only restored, but enhanced, fetal growth relative to sea level controls. Incubation at high altitude of sea level eggs with oxygen supplementation completely prevented the high altitude-induced fetal growth restriction. Thus, fetal oxygenation, independent of maternal nutrition during development, has a predominant role in the control of fetal growth. Further, prolonged high altitude residence confers protection against the deleterious effects of hypoxia on fetal growth.

\begin{abstract}
(Received 26 July 2007; accepted after revision 17 October 2007; first published online 25 October 2007)
Corresponding author D. A Giussani: Department of Physiology, Development and Neuroscience, University of Cambridge, Cambridge CB2 3EG, UK. Email: dag26@cam.ac.uk
\end{abstract}

It is generally accepted that hypoxia during pregnancy decreases birth weight; however, it has been difficult to demonstrate or isolate this effect because all human conditions or experimental animal models that induce fetal hypoxia are also accompanied by changes in nutrient delivery. For instance, elegant studies in mammalian animals have shown that maternal chronic hypoxia during pregnancy can lead to slow, disproportionate fetal growth (De Grauw et al. 1986; Jacobs et al. 1988). However, whether these effects are due to fetal under-oxygenation or fetal under-nutrition is uncertain as chronic experimental hypoxia also reduces maternal food intake (De Grauw et al. 1986). In humans, fetal hypoxia occurs most commonly under physiological conditions during the hypobaric hypoxia of pregnancy at high altitude (Moore, 1990), or under pathological

This study is dedicated to the memory of Professor Dr Lieselotte Bauer de Barragán, previous Director of the Hospital San Gabriel, who dedicated most of her life to the welfare of the mother and newborn at high altitude. The study received The Pfizer Award at the 52nd Annual meeting of the Society for Gynecologic Investigation. conditions during pregnancy complicated by placental insufficiency (Baschat, 2004). Several investigators have reported reduced birth weight and asymmetric growth restriction in babies with increasing altitude (e.g. Lichty et al. 1957; McClung, 1969; Haas et al. 1980; Moore et al. 1998; Giussani et al. 2001; Zamudio et al. 2007). However, because most high altitude populations are also impoverished, and because placental insufficiency decreases nutrient as well as oxygen transfer to the baby, the extent to which the reduction in fetal growth under these conditions is governed by fetal under-nutrition or under-oxygenation, again, remains uncertain. By using the chick embryo as an animal model, this study could isolate the direct effects on the fetus of developmental hypoxia due to high altitude for the first time, completely independent of changes in maternal nutrition and of the physiology of the mother or the placenta. The study tested the hypothesis that fetal oxygenation, independent of maternal nutrition during development, has a predominant role in the control of fetal growth by investigating the effects of incubation at high altitude of fertilized eggs laid by sea level hens. In addition, the experiment could be done the 
other way around, by developing at sea level fertilized eggs laid by high altitude hens, to assess whether the hypoxia-induced effects on fetal growth could be reverted, something that is difficult if not impossible to assess in human populations. Finally, to discount the possibility that high altitude-induced fetal growth restriction is due to hypobaria rather than hypoxia, the effects of incubation at high altitude with oxygen supplementation of fertilized eggs laid by sea level hens was also investigated.

\section{Methods}

The study was done in Bolivia, in the high altitude city of La Paz $\left(3600 \mathrm{~m}, 494 \mathrm{mmHg}\right.$, approximate ambient dry $P_{\mathrm{O}_{2}}$ $100 \mathrm{mmHg}$ ) and the sea level city of Santa Cruz (420 m, $760 \mathrm{mmHg}$, approximate ambient dry $P_{\mathrm{O}_{2}} 160 \mathrm{mmHg}$ ). Fertilized eggs were obtained from Black Leghorn chickens that had been reared at the sea level city of Santa Cruz or at high altitude city of $\mathrm{La} \mathrm{Paz}$ for at least six generations. Fertilized eggs from sea level hens, laid at sea level, were randomly divided and incubated either at sea level (SLSL, $n=45$ ) or high altitude (SLHA, $n=60$ ). Eggs from high altitude hens, laid at high altitude, were randomly divided and incubated either at high altitude (HAHA, $n=70$ ) or sea level (HASL, $n=50$ ). SLHA embryos were also incubated with oxygen supplementation (SLHA $+\mathrm{O}_{2}$, $n=42$ ) at rates to maintain sea level oxygen partial pressures according to Dalton's law (see West, 2004).

Egg storage is commonly practiced in the artificial incubation of domestic birds. If the storage temperature for freshly laid chicken eggs is kept below the physiological zero $\left(25-27^{\circ} \mathrm{C}\right)$, dormancy of the embryo can be maintained and fertile eggs can be stored for 1-3 weeks (Butler, 1991). In this study, fertilized eggs from any one group were accumulated, maintained and transported over $2-3$ days at $14^{\circ} \mathrm{C}$ to arrest and synchronize development, prior to incubation. Eggs were weighed prior to incubation. All incubations (Polyhatch, Brinsea Products Ltd, Sandford, UK) were carried under conditions to optimize development, with controlled temperature $\left(38^{\circ} \mathrm{C}\right)$, humidity $(60 \%)$ and appropriate egg rotation. On day 20 , out of the 21 day incubation period, the egg was weighed, the air cell was exposed and chorio-allantoic venous blood was drawn into a $1 \mathrm{ml}$ syringe for analysis of $P_{\mathrm{O}_{2}}$ (ABL 500; Radiometer, Copenhagen, Denmark) and haematocrit, whenever possible in duplicate (Hawksley centrifuge). Following killing by spinal transection, the fetus was removed from the eggshell and weighed. Head diameter and body length (crown rump length) were measured with a digital micrometer. The fetal brain was dissected and weighed.

Growth efficiency was calculated as the ratio of the 'measured fetal weight at day 20' and the product of 'the egg weight at day 20 ' minus 'the weight of the egg once the embryo had been removed at day 20'. An example, for a SLSL and a SLHA embryo is given in Scheme 1.

To calculate the partitioning of the resource, both the 'measured fetal weight at day 20 ' and 'egg weight at day 20 - the measured fetal weight at day 20 ' were expressed as a percentage of the egg weight at day 20 and plotted in histogram format. An example for the same SLSL and SLHA embryos as above is given in Scheme 2.

All procedures were approved by the local ethics committee (Consejo Tecnico) of the Bolivian Institute for High Altitude Biology (IBBA), Universidad Mayor de San Andrés, La Paz, Bolvia. All variables were analysed for normality of distribution and then expressed as means \pm s.E.M. Comparisons between groups were assessed statistically using One-way ANOVA with an appropriate post hoc test (SigmaStat; Systat Software Inc., San Jose, CA, USA). Differences in mortality were compared using a contingency table and assessed by the chi-square $\left(\chi^{2}\right)$ test. For all comparisons, statistical significance was accepted when $P<0.05$.

\section{Results}

The data show that incubations at high altitude increased embryonic mortality relative to incubations at sea level, but this effect was reduced in HAHA embryos. The altitude-induced increase in mortality in the SLHA group was prevented by oxygen supplementation (mortality: SLSL $=28.8 \%, \quad$ SLHA $=66.6 \% ; \quad$ HAHA $=52.8 \%$; HASL $=28.1 \%$; SLHA $+\mathrm{O}_{2}=30.2 \% ; P<0.05, \chi^{2}$ test $)$. Surviving chick embryos incubated at high altitude were hypoxic relative to chick embryos incubated at sea level, with the exception of those with oxygen supplementation,
SLSL

measured fetal body weight at day 20

(weight of egg at day 20 - weight of egg without embryo at day 20)

measured fetal body weight at day 20

SLHA

(weight of egg at day 20 - weight of egg without embryo at day 20)

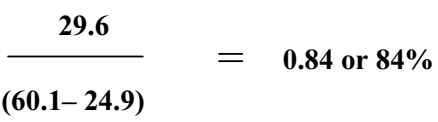

14.9

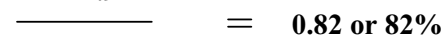

$(60.5-42.3)$

Scheme 1. 
SLSL

measured fetal body weight at day 20

(egg weight at day 20)
X 100

(egg weight at day 20 - measured fetal body weight at day 20)

(egg weight at day 20) x 100

$\frac{(60.1-29.6)}{(60.1)} \times 100=51 \%$ measured fetal body weight at day 20

(egg weight at day 20) x 100

SLHA

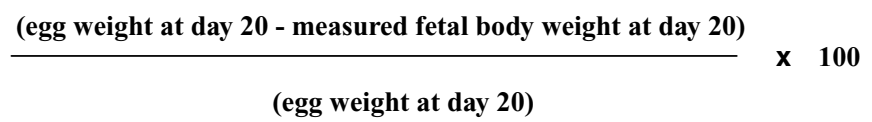

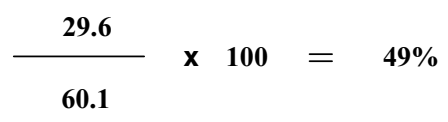

60.1

Scheme 2.

in which sea level oxygen partial pressure was maintained (Fig. 1A). Relative to SLSL and SLHA $+\mathrm{O}_{2}$ chick embryos, haematocrit was elevated in the SLHA, HAHA and HASL groups $(P<0.05)$, despite the latter group being incubated at sea level (Fig. 1B).

Prior to incubation, fertilized eggs from high altitude hens were lighter than fertilized eggs from sea level hens (Fig. 2A). At the end of the incubation period, in terms of absolute weight, chick embryos incubated at high altitude showed pronounced growth restriction, relative to chick embryos incubated at sea level $(P<0.05)$, with the exception of the SLHA $+\mathrm{O}_{2}$ group, in which the growth restriction was completely prevented (Fig. $2 B$ ). When embryonic weight was expressed as a percentage of the egg mass prior to incubation, to account for the initial differences in weights between high altitude and sea level eggs, fetal growth was restricted by $45.2 \%$ in the SLHA group and by $22.2 \%$ in the HAHA group relative to SLSL controls (Fig. 2C). Incubation at sea level of eggs laid by hens at high altitude not only restored growth, but these embryos grew heavier than the SLSL group. The embryonic growth restriction of incubations at high altitude was asymmetric as head growth was spared at the expense of body length (Fig. $3 A$ and $B$ ). Hence, the relative brain weight, expressed as a percentage of the fetal body mass, was elevated in embryo groups, which had suffered body growth restriction during incubations at high altitude (Fig. $3 C$ ). The ratio of the head diameter to body weight was elevated in chick embryos incubated at high altitude. However, the increase in this ratio was markedly attenuated in the HAHA chicks relative to the SLHA group (Fig. 3D). Oxygen supplementation completely prevented the high altitude-induced asymmetry in fetal growth (Figs 2 and 3). Calculation of growth efficiency and the partitioning of the resource showed that reductions in oxygen availability during incubations at high altitude did not affect how well the resource is converted into fetal body mass (growth efficiency) (Fig. 4A). Rather, growth restriction at high
A

Figure 1. Partial pressure of oxygen and haematocrit

Values are means + S.E.M. for chorioallantoic venous blood taken from sea level chick embryos incubated either at sea level (SLSL, open bar, $n=9)$ or high altitude (SLHA, filled bar, $n=12$ ), high altitude embryos incubated at high altitude (HAHA, stippled bar, $n=10$ ) or sea level (HASL, hatched bar, $n=7$ ), and from sea level chick embryos incubated at high altitude with oxygen supplementation (SLHA $+\mathrm{O}_{2}$, grey bar, $n=12$ ). Different letters are significantly different by one-way ANOVA with Student-Newman-Keuls test $(P<0.05)$.

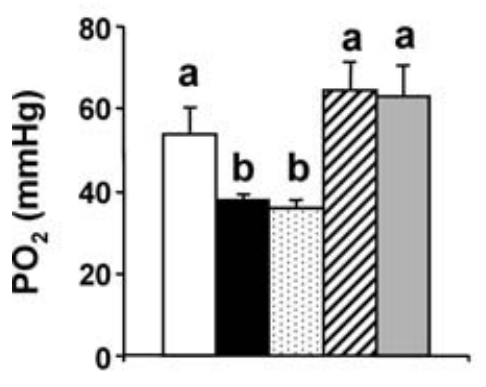

$B$

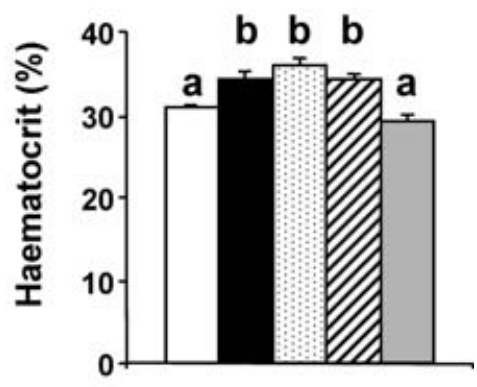



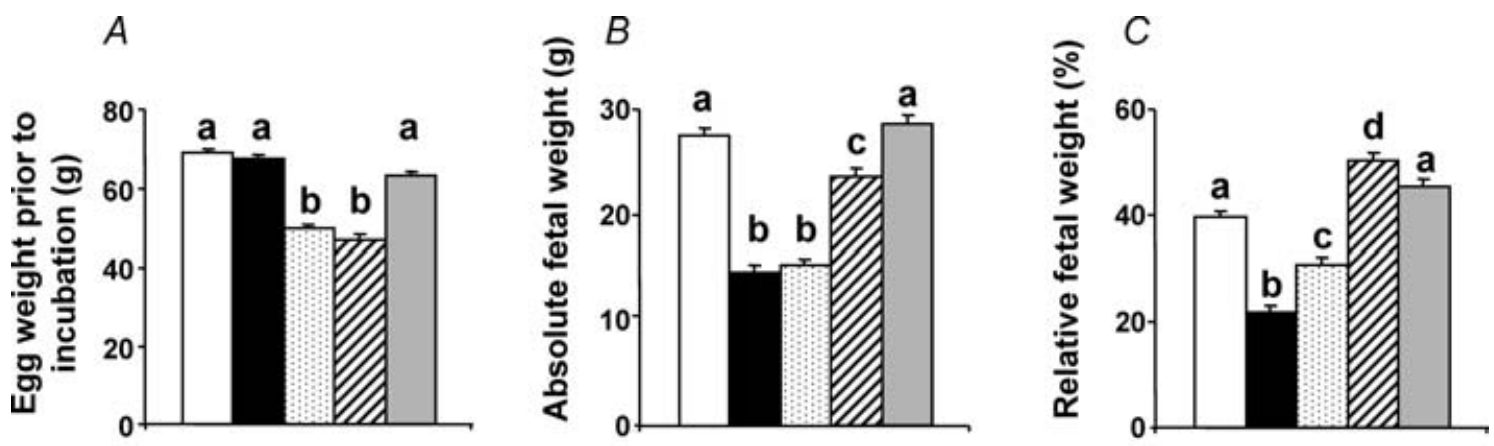

Figure 2. Fetal growth following high and lowland incubations

Values are means + S.E.M. for the egg weight prior to incubation $(A)$, the absolute fetal weight at the end of the incubation period $(B)$ and the fetal weight at the end of the incubation period expressed as a percentage of the initial egg mass (C). SLSL (open bar, $n=31$ ), SLHA (filled bar, $n=19$ ), HAHA (stippled bar, $n=33$ ), HASL (hatched bar, $n=30$ ), and SLHA $+\mathrm{O}_{2}$ (grey bar, $n=26$ ). Different letters are significantly different by one-way ANOVA with Student-Newman-Keuls or Dunn's tests, as appropriate $(P<0.05)$.

altitude was due to a reduction in fetal resource uptake (Fig. 4B).

\section{Discussion}

The data show that development of chick embryos at high altitude induced fetal hypoxaemia and led to an increase in fetal haematocrit. Whilst several studies have reported an increase in packed red cell mass in the umbilical blood of human infants following gestation at high altitude (Ballew \& Haas, 1986; Buys de Jorge et al. 1988; Leibson et al. 1989; Niermeyer et al. 1995; Ramirez-Cardich et al.
2004), this is the first direct demonstration that the partial pressure of oxygen is actually reduced in the fetal blood during development at high altitude. The data also show that incubation at high altitude of fertilized eggs laid by sea level hens restricted fetal growth, in similar fashion to restriction of fetal growth in the chick embryo by isobaric hypoxia (Miller et al. 2002). Incubation at high altitude of fertilized eggs laid by high altitude hens also restricted fetal growth, but to a lesser extent compared to eggs laid by sea level hens. By contrast, incubation at sea level of fertilized eggs laid by high altitude hens not only restored, but enhanced, fetal growth relative to sea level
A
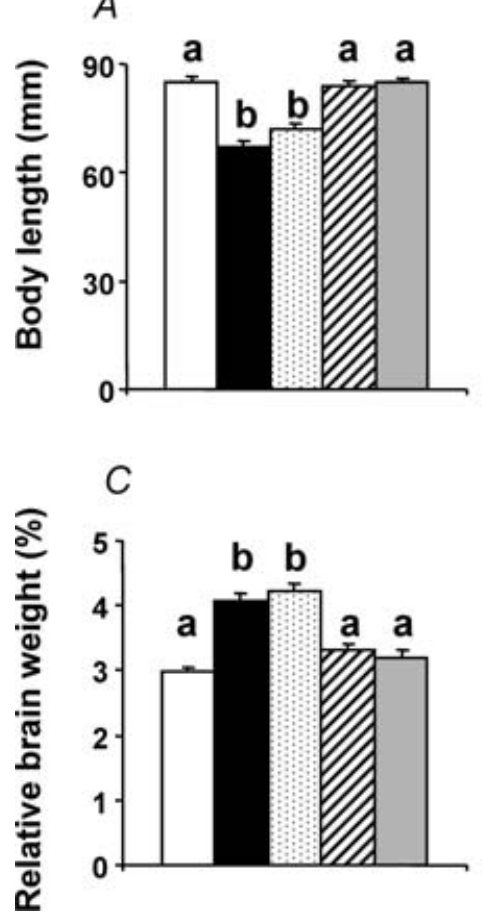

$B$
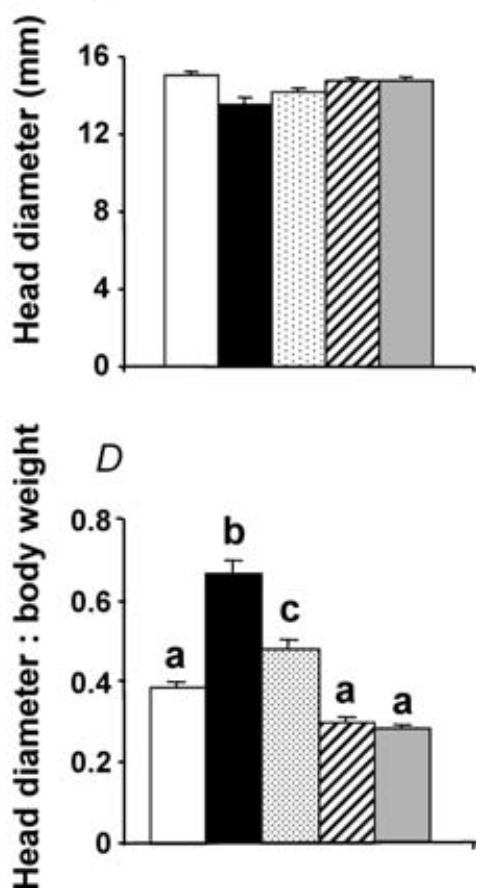

Figure 3. Symmetry of fetal growth following high and lowland incubations

Values are means + S.E.M. for the body length $(A)$, the head diameter $(B)$, the brain weight expressed as a percentage of the fetal body weight $(C)$ and the ratio of the head diameter to body weight $(D)$. SLSL (open bar, $n=31$ ), SLHA (filled bar, $n=19$ ), HAHA (stippled bar, $n=33$ ), HASL (hatched bar, $n=30$ ), and SLHA $+\mathrm{O}_{2}$ (grey bar, $n=26$ ). Different letters are significantly different by one-way ANOVA with Dunn's test $(P<0.05)$. 
controls. Incubation at high altitude of sea level eggs with oxygen supplementation completely prevented the high altitude-induced fetal growth restriction.

The data obtained from the chick embryo in the current study resembles that presented in an epidemiological study of human populations in Bolivia (Giussani et al. 2001). In that study, birth weight records were obtained from term pregnancies in La Paz and Santa Cruz, especially from obstetric hospitals attended by wealthy or impoverished mothers. The data revealed pronounced asymmetric growth restriction in babies born at high altitude relative to sea level. When lowland babies born from mothers with high or low economic status were compared, birth weight was significantly reduced in low versus high income groups, but this difference was not as pronounced as the effect on birth weight of high altitude alone. Additional data also showed that highland babies from poor families did not have the greatest reduction in birth weight, as one would have expected. Rather, counter-intuitively, these babies were actually heavier than highland babies born from families with a high socio-economic status. The apparent conundrum was easily explained by assessing the ancestry of the families. In that study, the low socio-economic group of $\mathrm{La} \mathrm{Paz}$ contained a high percentage $(92 \%)$ of women from Amerindian origin with Aymara indian paternal and maternal surnames. In contrast, the high socio-economic group of $\mathrm{La} \mathrm{Paz}$ contained a high European admixture. The findings of that study support the observations of Moore (1990) and Haas et al. (1980), who suggested that fetal growth restriction at altitude is correlated with the duration of high altitude residence: the longest resident population experiencing the least decline in birth weight with altitude, while the shortest historical residence groups, the greatest decline. Asymmetric growth restriction has been attributed, in part, to the sustained redistribution of the fetal cardiac output, to maintain oxygenation of essential circulations, such as those perfusing the brain, at the expense of the peripheral vascular beds (Akalin-Sel \& Campbell, 1992; Giussani et al. 1993). Interestingly, a report by Martyn et al. (1996) suggested that, in human babies, an increase in the ratio of the head circumference to birth weight is associated with increased stroke in adulthood. Data in the present manuscript show that incubation of chick embryos at high altitude also produced an elevated ratio of the head diameter to body weight, and that this effect was also reduced in embryos from high altitude hens. Combined, our experiments in the chick embryo and observations in human babies in Bolivia therefore suggest that oxygen deprivation, independent of maternal nutrition during development, has a predominant role in the control of fetal growth. Further, prolonged high altitude residence confers protection against the effects of hypoxia on fetal mortality, fetal growth and the developing cardiovascular system. The overwhelming similarities between our findings in human babies and chick embryos make a serious case for the applicability of experiments with chicken eggs to model human conditions.

The physiology underlying the protection conferred by prolonged high altitude residence against the deleterious effects of hypoxia on fetal development remains unknown. In humans, a comparison of blood flow in Tibetan and Han Chinese pregnant women living at high altitude showed a greater redistribution of blood flow from the common iliac artery to the uterine artery in Tibetan than Han women. Thus, delivery of uteroplacental oxygen was increased and heavier babies were noted, despite lower arterial oxygen content in Tibetan women (Moore et al. 2001). A recent study by Zamudio et al. (2007) tested the hypothesis that greater maternal uteroplacental oxygen delivery would also explain increased human fetal growth at altitude in Andeans versus Europeans in Bolivia. They concluded that genetically mediated differences in maternal oxygen delivery did not explain the Andean advantage. Rather, the mechanism underlying this protection likely resides within the feto-placental unit. The present findings in the chick embryo confirm this expectation since protection conferred by prolonged high altitude residence could also express itself directly at the fetal, rather than the maternal or uteroplacental, levels. One possibility may be adaptations that enhance fetal arterial oxygen saturation.
Figure 4. Use of resource for fetal growth during high and lowland incubations

Values are mean + S.E.M. for the growth efficiency $(A)$ and the partitioning of the resource $(B)$, both expressed as a percentage. SLSL (open bar, $n=31$ ), SLHA (filled bar, $n=19$ ), HAHA (stippled bar, $n=33$ ), HASL (hatched bar, $n=30$ ), and SLHA $+\mathrm{O}_{2}$ (grey bar, $n=26)$. Values for partitioning of resource (mean + S.E.M.) are expressed as a histogram on top of the corresponding group. Different letters are significantly different by one-way ANOVA + Student-NewmanKeuls or Dunn's tests, as appropriate $(P<0.05)$. For calculations with examples, see Methods.
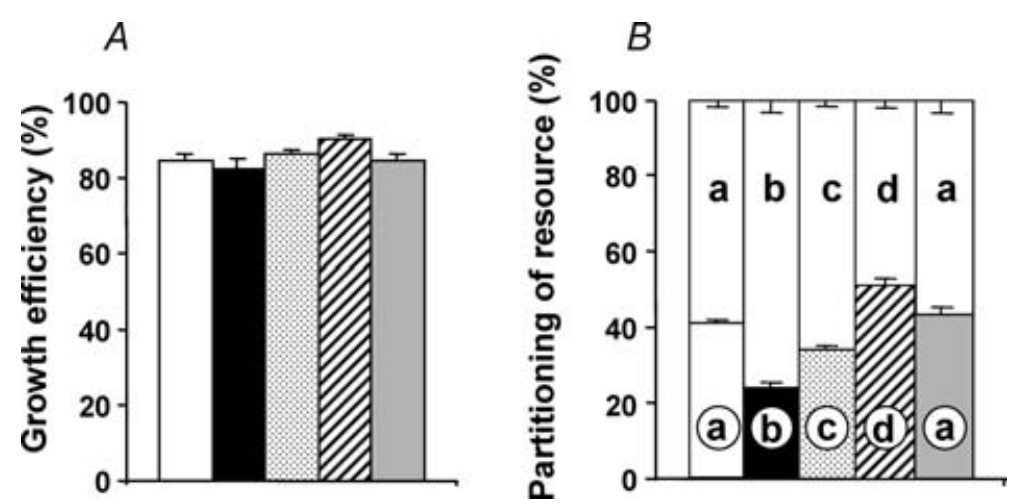
Whilst this has not been shown in the unborn child, an interesting study by (Beall, 2000) identified an autosomal major gene that confers higher resting oxygen saturation in sedentary Tibetan natives. In addition, Velarde et al. (1991) have also reported that Peruvian high altitude chickens have evolved a high haemoglobin-oxygen affinity, a finding suggested to be a genetic response to high altitude hypoxia. In our study, the highland chickens had lived at high altitude only for at least six generations. Whether this is sufficient time for physiological adaptations to high altitude to become genetically determined is questionable. However, the possibility exists that high altitude hypoxia may confer protection via epigenetic mechanisms, alterations in gene regulation that occur without a change in DNA sequence. Epigenetic marks such as CpG methylation and histone tail modifications have been shown to be affected by environmental conditions (Jirtle \& Skinner, 2007), and because epigenetic marks are heritable (see Godfrey et al. 2007), gene expression and physiological responses can be altered in a comparatively shorter time frame, from one generation to the other, and for years to come.

To give further insight into the physiological mechanisms by which high altitude hypoxia depresses fetal growth, and the protection against this effect in highland chickens, we asked two further questions: do alterations in oxygen availability during development affect how well the resource is used by the fetus? Or does oxygen availability affect how well the resource is converted into fetal body mass? Calculation of growth efficiency and the partitioning of the resource in all five groups of chick embryos revealed that high altitude hypoxia did not affect growth efficiency, but it had a marked effect on fetal resource uptake or resource utilization for tissue accretion. For instance, incubation at high altitude of embryos from sea level hens had the greatest depressive effect on fetal growth because these embryos had the least uptake of resource. By contrast, despite development under similar conditions of fetal hypoxia, fetal resource uptake was greater in HAHA compared to SLHA groups. The mechanism by which development under hypoxic conditions reduces resource utilization by the fetus remains unresolved, but it is likely to be due to the depressive effects of oxygen deprivation on ATP synthesis and/or due to the effects of hypoxia on endocrine factors important in the regulation of fetal growth, such as insulin, thyroxine, cortisol and insulin-like growth factors (IGFs; see Fowden, 1995). Elucidation of changes in fetal nutrient utilization imposed by high altitude hypoxic pregnancy in humans and other mammals would require calculation of maternal glucose delivery and umbilical glucose uptake. In experimental mammalian models, such as sheep, this is highly technical and requires the chronic implantation of catheters into the umbilical vein, fetal dorsal aorta and caudal vena cava, and into the uterine ovarian vein and the maternal aorta with simultaneous blood sampling during experimentation (Comline \& Silver, 1972). The simplicity with which answers to similar questions can be obtained in the chick embryo again highlights the excellence and applicability of this animal model.

A final component of the data presented in this study shows that when fertilized eggs laid by high altitude hens were incubated at sea level, the resulting embryos not only recovered their growth, but they grew heavier than sea level controls. The haematocrit data reveal that this group of embryos retained an increased oxygen carrying capacity despite incubation at sea level. This suggests that embryos from high altitude hens incubated at sea level had a greater oxygen content than sea level controls, further supporting a role for oxygen in the control of fetal growth. The mechanism by which elevated haematocrit levels are maintained in the absence of a hypoxic stimulus is unknown, but the data may reflect an adaptive response, transmitted by the mother to the oocyte prior to egg laying, predictive of fetal development in a hypoxic environment. Another example of a maternal predictive adaptive response (Gluckman \& Hanson, 2004) is that of the meadow vole, in which the photoperiodic history of the dam prior to conception, rather than the perinatal thermal environment, can better determine the offspring's coat thickness at birth (Lee \& Zucker, 1988). Alternatively, the maintained elevated haematocrit in HASL embryos may highlight that genetic or epigenetic control of factors determining oxygen carrying capacity is regulated very early on in the developmental process of the oocyte by the available oxygen concentration at that time.

In conclusion, we have isolated the effects of alterations in fetal oxygenation on fetal growth. Oxygen, independent of maternal nutritional factors, has a predominant role in the control of fetal growth and prolonged high altitude residence confers protection against the effects of hypoxia on fetal development. This discovery calls for the attention of this aspect of growth regulation not only in women undergoing pregnancy at high altitude, but also in sea level human pregnancies complicated with reduced oxygen delivery to the fetus, such as during placental insufficiency and pre-eclampsia (Many et al. 1996; Baschat, 2004).

\section{References}

Akalin-Sel T \& Campbell S (1992). Understanding the pathophysiology of intra-uterine growth retardation: the role of the 'lower limb reflex' in redistribution of blood flow. Eur J Obstet Gynecol Reprod Biol 46, 79-86.

Ballew C \& Haas JD (1986). Hematologic evidence of fetal hypoxia among newborn infants at high altitude in Bolivia. Am J Obstet Gynecol 155, 166-169.

Baschat AA (2004). Fetal responses to placental insufficiency: an update. BJOG 111, 1031-1041.

Beall CM (2000). Tibetan and Andean patterns of adaptation to high-altitude hypoxia. Hum Biol 72, 201-228. 
Butler DE (1991). Egg handling and storage at the farm and hatchery. In Avian Incubation, ed.Tullet SD, pp. 195-203. Butterworth, London.

Buys de Jorge MC, Contrini MA, Miranda C, Carrera C, Torrejon I, Martin B \& Scaro JL (1988). Sangre (Barc) 33, 97-101.

Comline RS \& Silver M (1972). The composition of foetal and maternal blood during parturition in the ewe. J Physiol 222, 233-256.

De Grauw TJ, Myers R \& Scott WJ (1986). Fetal growth in rats from different levels of hypoxia. Biol Neonate 49, 85-89.

Fowden AL (1995). Endocrine regulation of fetal growth. Reprod Fertil Dev 7, 351-363.

Giussani DA, Phillips PS, Anstee S \& Barker DJ (2001). Effects of altitude vs. economic status on birth weight and body shape at birth. Ped Res 49, 490-494.

Giussani DA, Spencer JA, Moore PJ, Bennet L \& Hanson MA (1993). Afferent and efferent components of the cardiovascular reflex responses to acute hypoxia in term fetal sheep. J Physiol 461, 431-449.

Gluckman PD \& Hanson MA (2004). Living with the past: Evolution, development, and patterns of disease. Science 305, 1733-1736.

Godfrey KM, Lillycrop KA, Burdge GC, Gluckamn PD \& Hanson MA (2007). Ped Res 61, 5R-10R.

Haas JD, Frongillo EF, Stepcik C, Beard J \& Hurtado L (1980). Altitude, ethnic and sex differences in birthweight and length in Bolivia. Hum Biol 52, 459-477.

Jacobs R, Robinson JS, Owens JA, Falconer J \& Webster MED (1988). The effect of prolonged hypobaric hypoxia on growth of fetal sheep. J Dev Physiol 10, 97-112.

Jirtle RL \& Skinner MK (2007). Environmental epigenomics and disease susceptibility. Nature Rev Genet 8, 253-262.

Lee TM \& Zucker I (1988). Vole infant development is influenced perinatally by maternal photoperiodic history. Am J Physiol Regul Integr Comp Physiol 255, R831-R838.

Leibson C, Brown M, Thibodeau S, Stevenson D, Vreman H, Cohen R, Clemons G, Callen W \& Moore LG (1989). Neonatal hyperbilirubinemia at high altitude. Am J Dis Child 143, 983-987.

Lichty JA, Ting RY, Bruns P \& Dyer E (1957). Studies of babies born at high altitude. 1 . Relation of altitude to birth weight. Am J Dis Child 93, 666-669.

Many A, Hubel CA \& Roberts JM (1996). Hyperuricemia and xanthine oxidase in preeclampsia, revisited. Am J Obstet Gynecol 174, 288-291.
Martyn CN, Barker DJP \& Osmond C (1996). Mothers' pelvic size, fetal growth, and death from stroke and coronary heart disease in men in the UK. Lancet 348, 1264-1268.

McClung J (1969). Effects of High Altitude on Human Birth. Harvard University Press, Cambridge, MA.

Miller SL, Green LR, Peebles DM, Hanson MA \& Blanco CE (2002). Effects of chronic hypoxia and protein malnutrition on growth in the developing chick. Am J Obstet Gynecol 186, 261-267.

Moore LG (1990). Maternal $\mathrm{O}_{2}$ transport and fetal growth in Colorado, Peru and Tibet high-altitude residents. Am J Hum Biol 2, 627-637.

Moore LG, Niermeyer S \& Zamudio S (1998). Human adaptation to high altitude: regional and life-cycle perspectives. Am J Phys Anthropol Suppl 27, 25-64.

Moore LG, Zamudio S, Zhuang J, Sun S \& Droma T (2001). Am J Phys Anthropol 114, 42-53.

Niermeyer S, Yang P, Shanmina, Drolkar, Zhuang J \& Moore LG (1995). Arterial oxygen saturation in Tibetan and Han infants born in Lhasa, Tibet. N Engl J Med 333, 1248-1252.

Ramirez-Cardich ME, Saito M, Gilman RH, Escate LE, Strouse JJ, Kabrhel C, Johnson C, Galchen R \& Bautista CT (2004). Effect of maternal anemia at high altitude on infant hematocrit and oxygenation. Am J Trop Med Hyg 70, 420-424.

Velarde FL, Espinoza D, Monge C \& de Muizon C (1991). A genetic response to high altitude hypoxia: high hemoglobin-oxygen affinity in chicken (Gallus gallus) from the Peruvian Andes. C R Acad Sci III 313, 401-406.

West JB (2004). Respiratory Physiology. The Essentials, 7th edn. Lippincott, Williams \& Wilkins, Baltimore.

Zamudio S, Postigo L, Illsley NP, Rodriguez C, Heredia G, Brimacombe M, Echalar L, Torricos T, Tellez W, Maldonado I, Balanza E, Alvarez T, Ameller J \& Vargas E (2007). Maternal oxygen delivery is not related to altitude- and ancestry-associated differences in human fetal growth. J Physiol 582, 883-895.

\section{Acknowledgements}

We thank Dr Wilma Tellez, Mr Armando Rodriguez, Mrs Martha Aguilar, Mrs Loyola Riveros, Mr Wilmar Velasquez and Mr Didi Maquera at IBBA, La Paz and Dr Ginella, Dr Nioshi and Dr Roca at CENETROP, Santa Cruz for their help with these studies. Financial support was provided by The British Heart Foundation and The Royal Society. 
Chapter III

Cardiac and vascular disease prior to hatching in chick embryos incubated at high altitude.

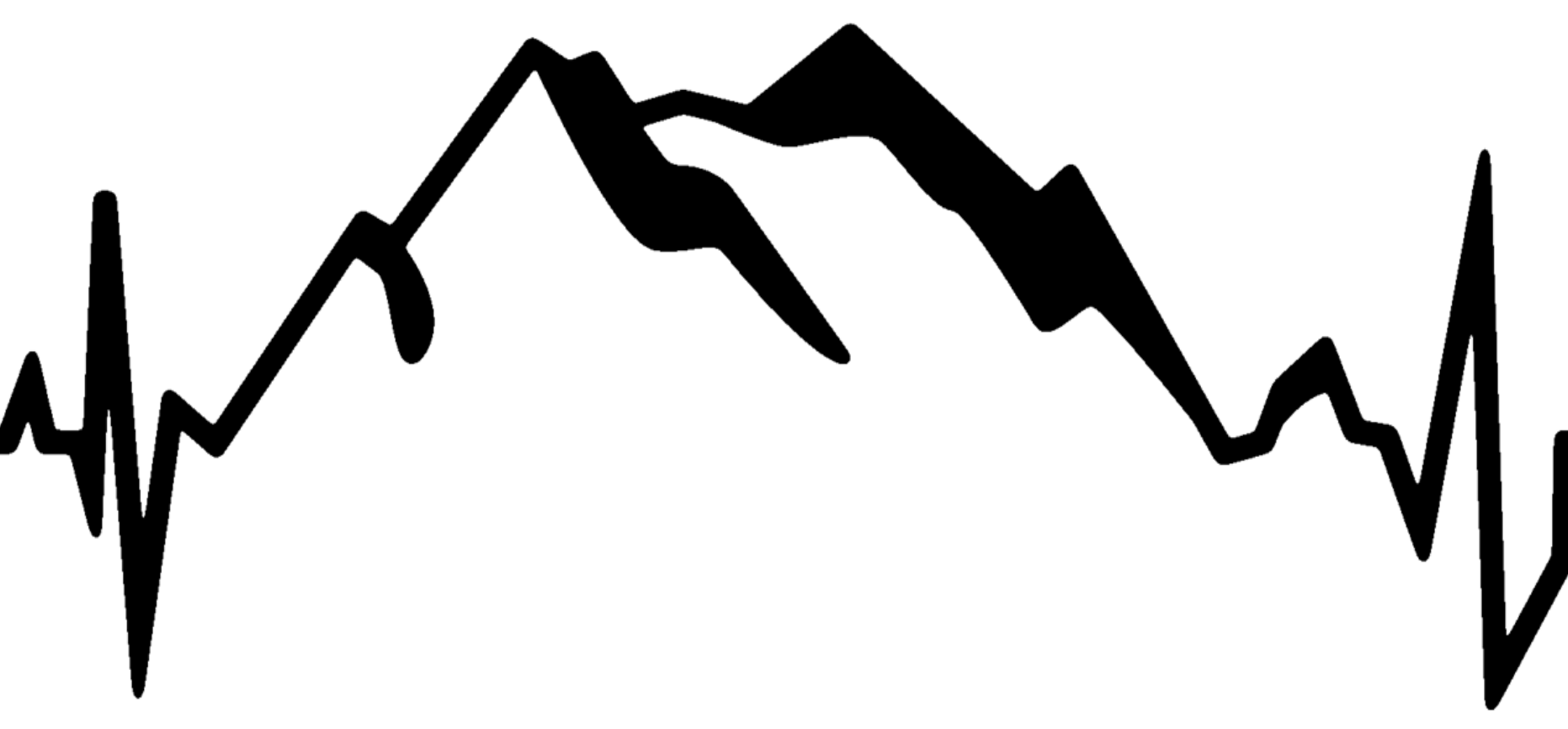

J Lev Orig Health Dis. 2010; 1:60-6 



\title{
Cardiac and vascular disease prior to hatching in chick embryos incubated at high altitude
}

\author{
C. E. Salinas ${ }^{1}$, C. E. Blanco ${ }^{2}$, M. Villena ${ }^{1}$, E. J. Camm ${ }^{3}$, J. D. Tuckett ${ }^{3}$, R. A. Weerakkody ${ }^{3}$, \\ A. D. Kane ${ }^{3}$, A. M. Shelley ${ }^{3}$, F. B. P. Wooding ${ }^{3}$, M. Quy ${ }^{3}$ and D. A. Giussani ${ }^{3 *}$ \\ ${ }^{1}$ Instituto Boliviano de Biología de Altura, Facultad de Medicina, Universidad Mayor de San Andrés, La Paz, Bolivia \\ ${ }^{2}$ Department of Pediatrics, Maastricht University, Maastricht, The Netherlands \\ ${ }^{3}$ Department of Physiology, Development and Neuroscience, University of Cambridge, Cambridge, UK
}

\begin{abstract}
The partial contributions of reductions in fetal nutrition and oxygenation to slow fetal growth and a developmental origin of cardiovascular disease remain unclear. By combining high altitude with the chick embryo model, we have previously isolated the direct effects of high-altitude hypoxia on growth. This study isolated the direct effects of high-altitude hypoxia on cardiovascular development. Fertilized eggs from sea-level or high-altitude hens were incubated at sea level or high altitude. Fertilized eggs from sea-level hens were also incubated at high altitude with oxygen supplementation. High altitude promoted embryonic growth restriction, cardiomegaly and aortic wall thickening, effects which could be prevented by incubating eggs from high-altitude hens at sea level or by incubating eggs from sea-level hens at high altitude with oxygen supplementation. Embryos from high-altitude hens showed reduced effects of altitude incubation on growth restriction but not on cardiovascular remodeling. The data show that: (1) high-altitude hypoxia promotes embryonic cardiac and vascular disease already evident prior to hatching and that this is associated with growth restriction; (2) the effects can be prevented by increased oxygenation; and (3) the effects are different in embryos from sea-level or high-altitude hens.
\end{abstract}

Received 28 May 2009; Revised 17 July 2009; Accepted 11 August 2009; First published online 1 October 2009

Key words: cardiovascular disease, chick embryo, high altitude, hypoxia.

\section{Introduction}

Despite healthy skepticism, ${ }^{1,2}$ evidence derived from human epidemiologic studies linking small size at birth with greater cardiovascular risk has gathered increasing support in recent years. ${ }^{3,4}$ This risk of cardiovascular disease not only results from intrauterine growth retardation in complicated pregnancy, but the association also extends across the normal range of birth weight in healthy pregnancy. ${ }^{1,3}$ A component of fetal growth is determined by the quality of the intrauterine environment. In turn, the quality of the intrauterine environment is largely determined by the available nutrient and oxygen supply to the growing young. Consequently, there have been many reports investigating the association between reduced fetal growth and increased risk of cardiovascular disease in animal models in which development has been complicated by reductions in fetal nutrition and/or in fetal oxygenation. ${ }^{5-10}$

Under physiologic conditions, in humans, fetal hypoxia occurs most commonly during the hypobaric hypoxia of pregnancy at high altitude. ${ }^{11}$ Although several investigators have reported reduced birth weight in human babies with increasing altitude, ${ }^{12-17}$ there have been no reports on the

*Address for Correspondence: Dr D. A. Giussani, Department of Physiology, Development and Neuroscience, University of Cambridge, Cambridge, CB2 3EG, UK.

(Emaildag26@cam.ac.uk) association between fetal growth restriction and alterations in cardiovascular development already evident prior to birth at high altitude in any species. Most high-altitude human populations are impoverished, therefore the extent to which any effects on fetal development during pregnancy at high altitude is governed by fetal under-nutrition or fetal underoxygenation, remains uncertain. By using the chick embryo as an animal model, an earlier study in our laboratory isolated the direct effects of developmental hypoxia owing to high altitude on embryonic growth, independent of changes in maternal nutrition and of the physiology of the mother or the placenta. ${ }^{18}$ The data in that study showed that high-altitude incubation of fertilized eggs laid by sea-level hens markedly restricted growth of the chick embryo. Incubation at high altitude of fertilized eggs laid by high-altitude hens also restricted embryonic growth, but to a lesser extent compared to eggs laid by sea-level hens. By contrast, incubation at sea level of fertilized eggs laid by high-altitude hens not only restored, but also enhanced growth relative to sea-level controls. Incubation at high altitude of sea-level eggs with oxygen supplementation completely prevented the high-altitudeinduced growth restriction. Thus, the oxygenation of the chick embryo, independent of maternal nutrition, has a predominant role in the control of its growth during development at high altitude. Further, prolonged high-altitude residence confers protection against the deleterious effects of hypoxia on growth. 
The present study tested the hypothesis that development at high altitude is related to a prenatal origin of cardiovascular disease and that hypoxia is the mechanism underlying the relationship. The hypothesis was tested three-fold: (1) by investigating the effects on the cardiovascular development of fertilized eggs laid by sea-level hens when incubated at high altitude; (2) by investigating whether alterations in the embryonic cardiovascular system induced by development at high altitude could be prevented by incubation at sea level of fertilized eggs laid by high-altitude hens; and (3) by investigating whether alterations in the embryonic cardiovascular system induced by development at high altitude could be prevented by incubation at high altitude of sea-level eggs with oxygen supplementation. We were also interested in whether prolonged high-altitude residence conferred any protection against any deleterious effects of hypoxia on cardiovascular development.

\section{Methods}

The study was done in Bolivia, in the high-altitude city of La $\mathrm{Paz}\left(3600 \mathrm{~m}, 494 \mathrm{mmHg}\right.$, approximate ambient dry $\mathrm{PO}_{2}$ $100 \mathrm{mmHg}$ ) and the sea-level city of Santa Cruz $(420 \mathrm{~m}$, $760 \mathrm{mmHg}$, approximate ambient dry $\mathrm{PO}_{2} 160 \mathrm{mmHg}$ ). The incubation procedures have been published earlier in detail. ${ }^{18}$ In brief, fertilized eggs were obtained from Black Leghorn chickens that had been reared at the sea-level city of Santa Cruz or at the high-altitude city of $\mathrm{La} \mathrm{Paz} \mathrm{for} \mathrm{at} \mathrm{least} \mathrm{six}$ generations. Fertilized eggs from sea-level hens, laid at sea level, were randomly divided and incubated either at sea level (SLSL, $n=31$ ) or high altitude (SLHA, $n=19$ ). Eggs from high-altitude hens, laid at high altitude, were randomly divided and incubated either at high altitude (HAHA, $n=33$ ) or sea level (HASL, $n=25$ ). SLHA embryos were also incubated with oxygen supplementation (SLHA $+\mathrm{O}_{2}$, $n=21$ ) at rates to maintain sea-level oxygen partial pressures according to Dalton's Law. ${ }^{19}$

All incubations (Polyhatch; Brinsea Products Ltd, UK) were carried under conditions to optimize development, with controlled temperature $\left(38^{\circ} \mathrm{C}\right)$, humidity $(60 \%)$ and appropriate egg rotation. On day 20, out of the 21-day incubation period, the egg was weighed, the air cell was exposed and chorioallantoic venous blood was drawn into a $1 \mathrm{ml}$ syringe for analysis of $\mathrm{PO}_{2}$ (ABL 500; Radiometer, Copenhagen, Denmark), whenever possible in duplicate. Following euthanasia by spinal transection, the embryo was removed from the eggshell and weighed. Head diameter and body length (crown-rump length) were measured with a digital micrometer.

The embryonic heart was dissected and weighed. In a subset of animals, following maximal dilatation using ethylenediaminetetraacetic acid (EDTA; $50 \mathrm{mg} / \mathrm{kg}$ ), a $5 \mathrm{~mm}$ segment of the thoracic aorta was dissected at the level of the apex of the heart, and the heart and aortic segment were fixed in $4 \%$ phosphate buffered paraformaldehyde for $24 \mathrm{~h}$ and then stored in physiologic buffer. Hearts and vessels were then embedded in paraffin. To account for possible shrinkage because of paraffin processing, the diameter of erythrocytes in heart sections was measured and compared to that obtained by measuring fresh erythrocytes from chick embryos at the same stage of incubation. ${ }^{20}$ All measurements were corrected using this factor. Mid-cardiac 4- $\mu \mathrm{m}$ coronal sections and $7-\mu \mathrm{m}$ transverse aortic sections were stained with van Gieson's solution. Slices were digitally recorded and analyzed by computerized morphometric systems (Quantimet 570; Leica, The Netherlands and Hauppauge Computer Works, UK).

All procedures were approved by the local ethics committee of the Bolivian Institute for High Altitude Biology (Consejo Técnico, IBBA, Universidad Mayor de San Andrés, La Paz, Bolivia). Comparisons between groups were assessed statistically using one-way ANOVA with the Student-Newman-Keuls post-hoc test (Sigma-Stat; SPSS Inc., Chicago, IL, USA). The relationships between indices of cardiovascular remodeling and embryonic size or $\mathrm{PO}_{2}$ were assessed using the Pearson ProductMoment correlation. A comparison between the slopes and intercepts of regression lines was conducted according to Armitage and Berry. ${ }^{21}$ For all comparisons, statistical significance was accepted when $P<0.05$.

\section{Results}

\section{Oxygenation and biometry in the chick embryo}

Analysis of this subset of animals confirms that incubation at high altitude induced embryonic systemic hypoxia and growth restriction (Table 1 ). The embryonic growth restriction is disproportionate as the ratio of the head diameter to body length was increased following incubation at high altitude (Table 1). When weight was expressed as a percentage of the initial egg mass, HAHA embryos showed partial protection against the effects of high-altitude incubation on growth. Further, the relative body weight in HASL embryos was greater than any other group (Table 1).

\section{Cardiac measurements in the chick embryo}

Relative to SLSL chick embryos, SLHA and HAHA groups showed significant increases of similar magnitude in the relative cardiac weight, and in the relative wall thickness of the left and right ventricles and septum (Fig. 1 and Table 2). In contrast, HASL and SLHA $+\mathrm{O}_{2}$ embryos had cardiac measurements similar to SLSL embryos (Fig. 1). However, the relative thickness of the walls of the left and right ventricles was significantly reduced when compared to all other groups in SLHA $+\mathrm{O}_{2}$ embryos (Fig. 1).

\section{Aortic measurements in the chick embryo}

SLHA embryos showed significant aortic medial thickening, as indexed by calculation of the aortic wall to lumen area ratio (Fig. 2). Aortae from HAHA embryos had the greatest wall 
Table 1. Oxygenation and biometry in the chick embryo

\begin{tabular}{llccc}
\hline & $\mathrm{PO}_{2}(\mathrm{mmHg})$ & Absolute body weight $(\mathrm{g})$ & Relative body weight (\%) & Head diameter : body length \\
\hline SLSL & $56 \pm 6(9)^{\mathrm{a}}$ & $28 \pm 1(31)^{\mathrm{a}}$ & $40 \pm 1(31)^{\mathrm{a}}$ & $0.177 \pm 0.001(31)^{\mathrm{a}}$ \\
SLHA & $38 \pm 2(12)^{\mathrm{b}}$ & $15 \pm 1(19)^{\mathrm{b}}$ & $22 \pm 1(19)^{\mathrm{b}}$ & $0.202 \pm 0.005(19)^{\mathrm{b}}$ \\
HAHA & $36 \pm 2(10)^{\mathrm{b}}$ & $15 \pm 1(33)^{\mathrm{b}}$ & $31 \pm 1(33)^{\mathrm{c}}$ & $0.201 \pm 0.003(33)^{\mathrm{b}}$ \\
HASL & $64 \pm 9(7)^{\mathrm{a}}$ & $24 \pm 1(25)^{\mathrm{c}}$ & $55 \pm 2(25)^{\mathrm{d}}$ & $0.176 \pm 0.001(25)^{\mathrm{a}}$ \\
SLHA $+\mathrm{O}_{2}$ & $64 \pm 4(10)^{\mathrm{a}}$ & $30 \pm 1(21)^{\mathrm{a}}$ & $45 \pm 1(21)^{\mathrm{a}}$ & $0.173 \pm 0.003(21)^{\mathrm{a}}$ \\
\hline
\end{tabular}

Values are mean \pm SEM for the partial pressure of oxygen in chorio-allantoic venous blood, the embryonic weight expressed as a percentage of the initial egg mass and the ratio of the head diameter to crown-rump length in sea-level chick embryos incubated either at sea level (SLSL) or high altitude (SLHA), high-altitude embryos incubated at high altitude (HAHA) or sea level (HASL), and from sea-level chick embryos incubated at high altitude with oxygen supplementation (SLHA $\left.+\mathrm{O}_{2}\right)$.

Number $(n)$ of chicks for each variable in parentheses.

${ }_{\mathrm{a}, \mathrm{b}, \mathrm{c}, \mathrm{d}}$ Values within columns that have different letters as superscripts are significantly different from each other (one-way ANOVA with Student-Newman-Keuls test; $P<0.05)$.
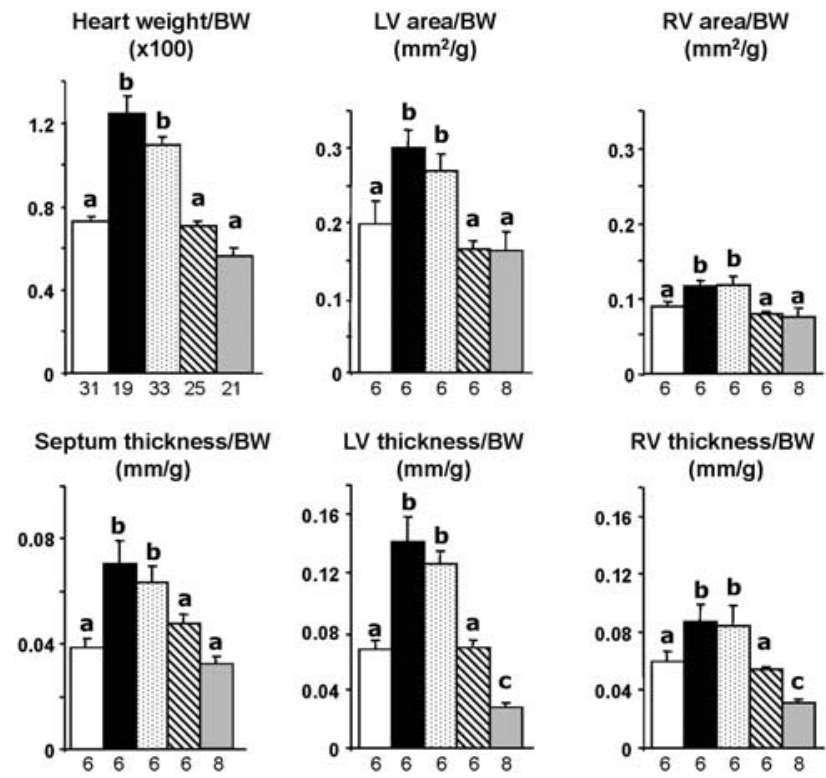

Fig. 1. Bars represent the mean \pm SEM for the heart weight, area and thickness of the walls of the left and right ventricles and thickness of the cardiac septum expressed relative to body weight in sea-level chick embryos incubated either at sea level (SLSL, open bar) or high altitude (SLHA, filled bar), high-altitude embryos incubated at high altitude (HAHA, stippled bar) or sea level (HASL, hatched bar), and in sea-level chick embryos incubated at high altitude with oxygen supplementation (SLHA $+\mathrm{O}_{2}$, gray bar). $n$ of each group for each variable are shown at the bottom of the histograms. Values within columns that have different letters as superscripts are significantly different from each other (one-way ANOVA with Student-Newman-Keuls test; $P<0.05$ ).

thickening relative to all other groups, as indexed by significant differences in all aortic measurements and derived calculations (Table 3 and Fig. 2). HASL or SLHA $+\mathrm{O}_{2}$ prevented the aortic thickening induced by incubation at high altitude (Fig. 2).

\section{Relation between cardiovascular remodeling and embryonic size or $\mathrm{PO}_{2}$}

Correlation analysis revealed that the embryonic body weight and $\mathrm{PO}_{2}$ were negatively related to the aortic wall to lumen area ratio in all groups independent of treatment (Fig. 3). By contrast, the ratio of the embryonic head diameter to body length was positively related to the aortic wall to lumen area ratio in all groups independent of treatment (Fig. 3). When body weight was related to the cardiac weight across all groups, the association was best described by a reverse exponential $\left(y=1.8951 \mathrm{e}^{-0.036 \mathrm{x}}, r=0.85\right)$ (Fig. 4a). Though SLSL, HASL and SLHA $+\mathrm{O}_{2}$ embryos were distributed across the right-hand side, SLHA and HAHA groups were distributed across the left-hand side of the association (Fig. 4a). The relation between body weight and cardiac weight remained significant even across the normal range for body weight in SLSL embryos (Fig. 4b). Though the embryonic body and cardiac weights were obtained in every chick, only organs from smaller subgroups of embryos were prepared for histology. Similarly, chorio-allantoic $\mathrm{PO}_{2}$ was obtained only from subgroups of embryos. Therefore, the relationship between embryonic body weight and any variable other than cardiac weight (for instance aortic wall to lumen area ratio or $\mathrm{PO}_{2}$ ) within any one group could not be investigated.

\section{Discussion}

Several experimental techniques, employed primarily in pregnant sheep, rats and guinea pigs, have been used to induce sustained fetal hypoxemia, including reductions in uterine and umbilical blood flow, ${ }^{22-25}$ placental embolization, ${ }^{26}$ pre-conceptual removal of endometrial caruncles ${ }^{27,28}$ and maternal chronic hypoxia. ${ }^{29-31}$ All these elegant studies have reported marked effects on the developing cardiovascular system. More recently, attention has focused on whether sustained prenatal hypoxia may have adverse consequences for 
Table 2. Cardiac measurements in the chick embryo

\begin{tabular}{|c|c|c|c|c|c|c|}
\hline & $\begin{array}{c}\text { Heart } \\
\text { weight (mg) }\end{array}$ & $\begin{array}{l}\mathrm{LV} \text { area } \\
\left(\mathrm{mm}^{2}\right)\end{array}$ & $\begin{array}{l}\text { RV area } \\
\left(\mathrm{mm}^{2}\right)\end{array}$ & $\begin{array}{l}\text { Septum } \\
\text { thickness (mm) }\end{array}$ & $\begin{array}{c}\text { LV wall } \\
\text { thickness }(\mathrm{mm})\end{array}$ & $\begin{array}{c}\text { RV wall } \\
\text { thickness }(\mathrm{mm})\end{array}$ \\
\hline SLSL & $196 \pm 4(31)$ & $5.5 \pm 0.8(6)$ & $2.2 \pm 0.2(6)$ & $1.11 \pm 0.05(6)$ & $1.87 \pm 0.16(6)$ & $1.59 \pm 0.17(6)$ \\
\hline SLHA & $185 \pm 7(19)$ & $4.6 \pm 0.2(6)$ & $1.8 \pm 0.2(6)$ & $1.10 \pm 0.05(6)$ & $2.13 \pm 0.07(6)$ & $1.32 \pm 0.07(6)$ \\
\hline НАНА & $175 \pm 5(33)$ & $4.2 \pm 0.5(6)$ & $1.8 \pm 0.3(6)$ & $1.02 \pm 0.05(6)$ & $2.08 \pm 0.09$ & $1.31 \pm 0.07(6)$ \\
\hline HASL & $188 \pm 6(25)$ & $4.0 \pm 0.3(6)$ & $1.8 \pm 0.1$ & $1.00 \pm 0.06(6)$ & $1.74 \pm 0.07(6)$ & $1.47 \pm 0.07(6)$ \\
\hline $\mathrm{SLHA}+\mathrm{O}_{2}$ & $194 \pm 9(21)$ & $5.0 \pm 0.6(8)$ & $2.3 \pm 0.3(8)$ & $1.01 \pm 0.09$ & $0.93 \pm 0.15$ & $1.08 \pm 0.07(8)$ \\
\hline
\end{tabular}

Values are mean \pm SEM for absolute cardiac measurements in sea-level chick embryos incubated either at sea level (SLSL) or high altitude (SLHA), high-altitude embryos incubated at high altitude (HAHA) or sea level (HASL), and from sea-level chick embryos incubated at high altitude with oxygen supplementation (SLHA $+\mathrm{O}_{2}$ ).

Number $(n)$ of chicks for each variable in parentheses.

${ }^{*} P<0.05 v$. SLSL, ANOVA + Student-Newman-Keuls test.

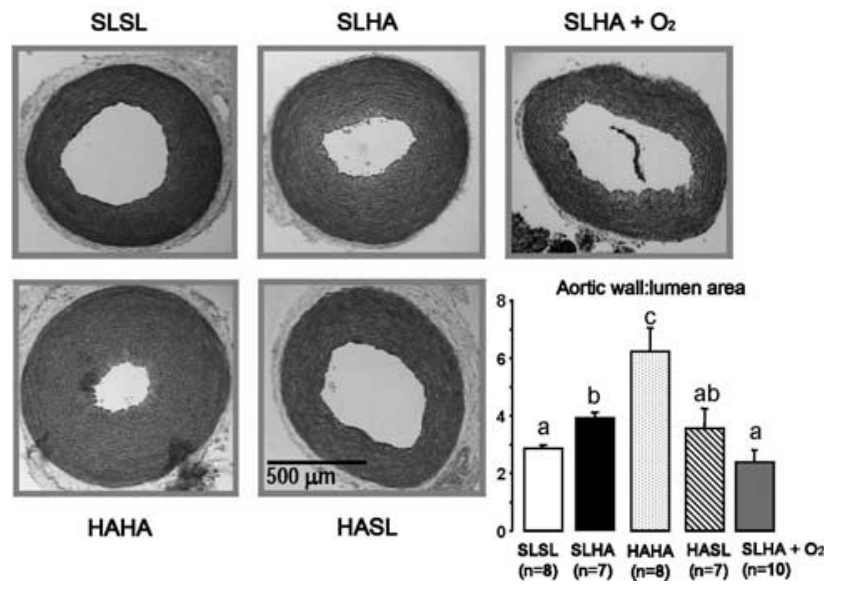

Fig. 2. Photomicrographs of representative examples of aortic sections and the mean \pm SEM of the aortic wall to lumen area ratio for sea-level chick embryos incubated either at sea level (SLSL, open bar) or high altitude (SLHA, filled bar), high-altitude embryos incubated at high altitude (HAHA, stippled bar) or sea level (HASL, hatched bar), and in sea-level chick embryos incubated at high altitude with oxygen supplementation (SLHA $+\mathrm{O}_{2}$, gray bar). Values within columns that have different letters as superscripts are significantly different from each other (one-way ANOVA with Student-Newman-Keuls test; $P<0.05$ ).

the function of the cardiovascular system after birth and in later life. For instance, the groups of Zhang, ${ }^{8}$ McMillen and Davidge $^{32-34}$ have reported that pregnant dams exposed to chronic hypoxia produce offspring with unequivocal cardiac and vascular dysfunction. However, because placental insufficiency decreases the delivery of nutrients as well as oxygen to the fetus and because chronic maternal hypoxia decreases maternal food intake, ${ }^{32-35}$ the extent to which the effects on the developing cardiovascular system of all the above interventions are because of fetal under-nutrition or underoxygenation remains uncertain. Employing the chick embryo as an animal model, a few studies have been able to isolate the effects on the developing cardiovascular system of chronic hypoxia, independent of changes in maternal nutrition and of the physiology of the mother and the placenta. Studies by Blanco and colleagues ${ }^{36}$ and the group of le Noble ${ }^{37}$ have confirmed that oxygen deprivation can act alone to remodel the developing cardiovascular system. Incubation of chick embryos with isobaric hypoxia induced embryonic aortic hypertrophic growth, left ventricular dysfunction and sympathetic hyperinnervation of peripheral arteries. ${ }^{36-39}$ The present study combined the use of the chick embryo model with incubation at high altitude to determine for the first time: (1) whether chronic hypoxia during development at high altitude is the mechanism underlying the relationship between growth restriction and cardiovascular disease already evident prior to hatching; (2) whether such effects are different in embryos from sea-level or high-altitude hens; and (3) whether the effects could be prevented by incubation of fertilized eggs from sea-level hens at high altitude with oxygen supplementation, or by incubation of fertilized eggs from high-altitude hens at sea level.

Analysis of this subset of animals confirms that incubation at high altitude induced embryonic growth restriction and that this effect was diminished in embryos from high-altitude hens. These findings support the observations of other studies reporting that in human populations prolonged high-altitude residence ancestry can confer protection against the effects of high altitude on fetal growth. ${ }^{1,14,16}$ The cardiovascular data in the present study show that incubation at high altitude leads to cardiac and aortic wall thickening in the chick embryo, independent of highland ancestry. Such cardiac and vascular remodeling could be prevented by incubation at sea level of fertilized eggs laid by high-altitude hens, or by incubation at high altitude of sea-level eggs with oxygen supplementation. Significant negative relationships were obtained between embryonic body weight or chorio-allantoic venous $\mathrm{PO}_{2}$ (equivalent to umbilical venous $\mathrm{PO}_{2}$ in mammalian pregnancy) with aortic wall thickening, and a significant positive relationship occurred between the ratio of 
Table 3. Aortic measurements in the chick embryo

\begin{tabular}{lccccc}
\hline & $\begin{array}{c}\text { Outer diameter } \\
(\mu \mathrm{m})\end{array}$ & $\begin{array}{c}\text { Lumen diameter } \\
(\mu \mathrm{m})\end{array}$ & $\begin{array}{c}\text { Wall thickness } \\
(\mu \mathrm{m})\end{array}$ & $\begin{array}{c}\text { Wall thickness/ } \\
\text { lumen radius ratio }\end{array}$ & $\begin{array}{c}\text { Wall area } \\
\left(\mathrm{mm}^{2}\right)\end{array}$ \\
\hline SLSL & $995 \pm 63$ & $508 \pm 30$ & $244 \pm 17$ & $0.96 \pm 0.03$ & $\begin{array}{c}\text { Lumen area } \\
\left(\mathrm{mm}^{2}\right)\end{array}$ \\
SLHA & $1085 \pm 39$ & $488 \pm 18$ & $288 \pm 14$ & $1.14 \pm 0.05$ & $727 \pm 57$ \\
HAHA & $1036 \pm 39$ & $396 \pm 24^{*}$ & $320 \pm 16^{*}$ & $1.66 \pm 0.14^{*}$ & $794 \pm 58$ \\
HASL & $996 \pm 82$ & $464 \pm 36$ & $266 \pm 29$ & $1.16 \pm 0.12$ & $637 \pm 108$ \\
SLHA $+\mathrm{O}_{2}$ & $939 \pm 68$ & $515 \pm 38$ & $212 \pm 28$ & $0.85 \pm 0.13$ & $500 \pm 85$ \\
\hline
\end{tabular}

Values are mean \pm SEM for aortic measurements in sea-level chick embryos incubated either at sea level (SLSL, $n=8$ ) or high altitude (SLHA, $n=7$ ), high-altitude embryos incubated at high altitude (HAHA, $n=8$ ) or sea level (HASL, $n=7$ ), and from sea-level chick embryos incubated at high altitude with oxygen supplementation (SLHA $\left.+\mathrm{O}_{2}, n=10\right)$.

${ }^{*} P<0.05 v$. SLSL, ANOVA + Student-Newman-Keuls test.
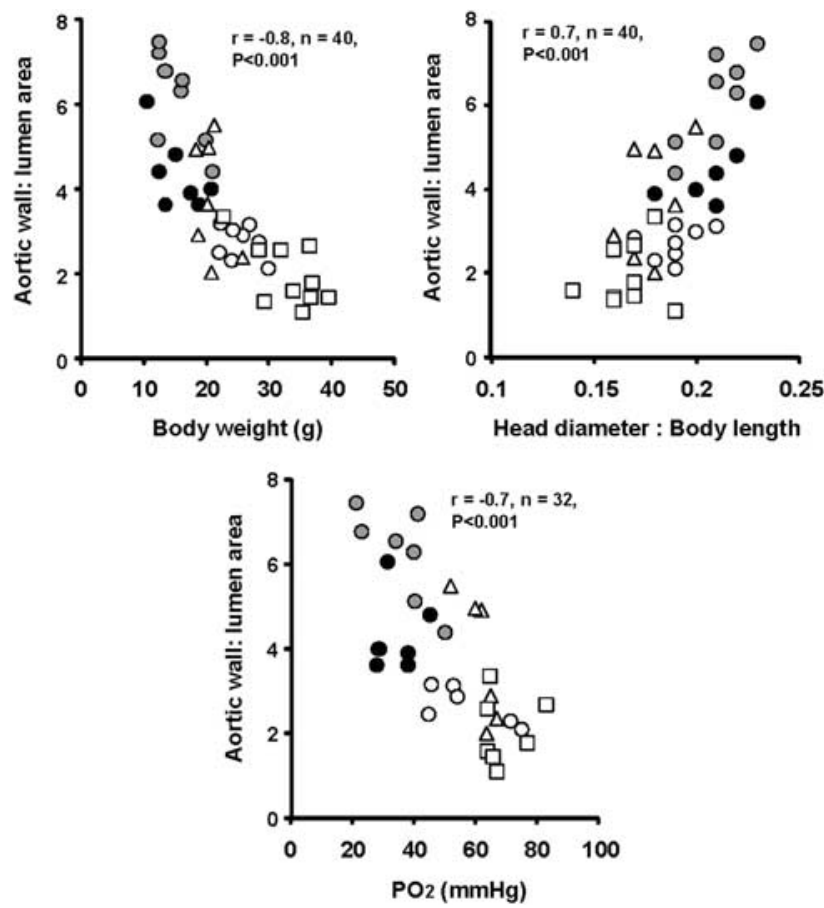

Fig. 3. Relationship between cardiovascular remodeling and embryonic size or $\mathrm{PO}_{2}$. Body weight, the head diameter to body length ratio and chorio-allantoic venous $\mathrm{PO}_{2}$ at the end of the incubation period were related to the aortic wall to lumen area ratio in all embryos independent of treatment. $r$, Pearson ProductMoment correlation coefficient; $n$, number of observations.

SLSL (O); SLHA ( $)$; HAHA (gray circles); HASL $(\triangle)$ and $\mathrm{SLHA}+\mathrm{O}_{2}(\square)$.

the embryonic head diameter to body weight (an index of growth symmetry) and the vascular changes. Combined, therefore, the data presented strongly implicate that hypoxia owing to high altitude is an important mechanism, retarding embryonic growth as well as triggering a developmental origin of cardiovascular disease, already evident prior to hatching/ birth. Interestingly, when body weight was related to cardiac weight, data in the present study also show that: (1) a significant negative relationship occurs across the normal range of weights; (2) that this relationship is shifted to the left and upwards by developmental high-altitude hypoxia; and (3) that the shift of the relationship could be restored by incubation at sea level of eggs from high-altitude hens, or by incubation at high altitude of sea-level eggs with oxygen supplementation. These observations have many commonalities with the original findings of Barker and colleagues, ${ }^{1,3}$ who related birth weight with increased rates of cardiovascular disease in human populations. They also reported a phenotypic association between asymmetric fetal growth restriction and cardiovascular risk factors, and that this relationship extended across the normal range of birth weights. ${ }^{1,3}$

There is general agreement that cardiovascular remodeling of this type results from an increase in peripheral resistance. $^{26,29,37}$ The aortic thickening may be a response to restore wall stress, as is typical of an increase in load, and the ventricular wall thickening occurs in response to the increased cardiac afterload. ${ }^{35,36}$ The hemodynamic overload may increase protein synthesis via a plethora of cellular and molecular pathways, including activation of stretch receptors, proto-oncogenes and vascular growth trophic factors. ${ }^{40,41}$ Hypoxia may also affect hypoxia-sensitive growth factors, such as VEGF (vascular endothelial growth factor). ${ }^{39,42}$ Consistent with the idea that this cardiovascular remodeling results from an increase in peripheral resistance, it has been reported that chronic hypoxia in the chick embryo promotes sympathetic hyper-innervation and enhanced norepinephrine release from perivascular sympathetic nerves; ${ }^{36,37}$ that it decreases NO-dependent relaxation; and that it increases constrictor reactivity in the peripheral vasculature. ${ }^{36}$

The data presented using the chick embryo model are of important human relevance. Three separate clinical studies ${ }^{43-45}$ have reported that babies born from pregnancies complicated by placental insufficiency show aortic thickening with increased vascular stiffness and reduced distensibility. A component of aortic thickening in the human fetus in pregnancies complicated by placental insufficiency may therefore be triggered by developmental hypoxia alone. 

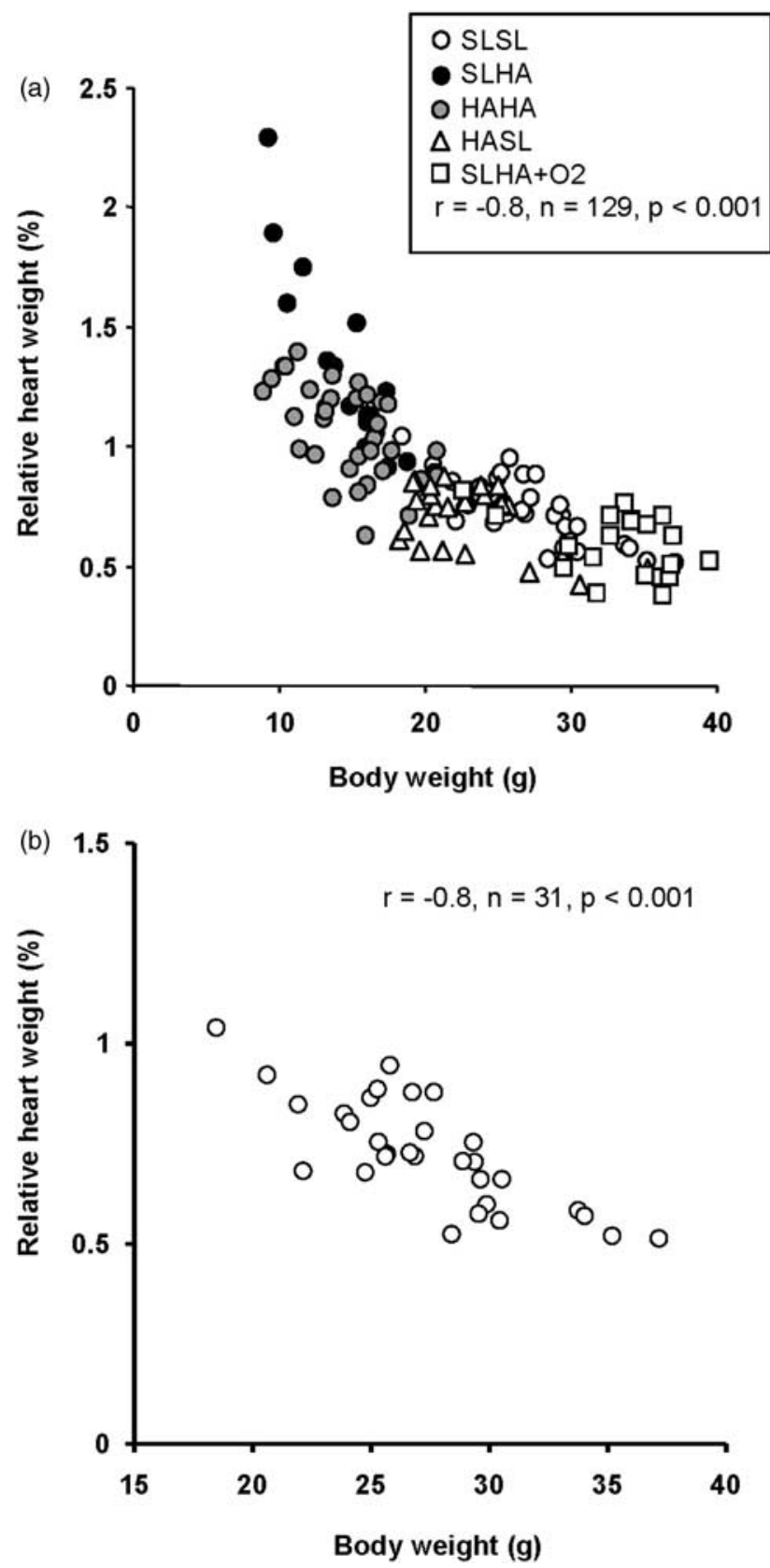

Fig. 4. Relationship between the cardiac weight and body weight in embryos following incubations at sea level and at high altitude $(r$, Pearson Product-Moment correlation coefficient; $n$, number of observations): (a) shows that a significant negative relationship occurs across all groups independent of treatment $(P<0.001)$; and $(b)$ shows that a significant negative relationship occurs across the normal range of weights in SLSL embryos $(P<0.001)$.

In conclusion, the data show that hypoxia owing to high altitude induces pronounced cardiovascular changes associated with disease in the chick embryo, which are already evident by the end of the incubation period, and that these cardiovascular alterations are associated with disproportionate growth restriction. The effects can be prevented by incubation at sea level of fertilized eggs laid by high-altitude hens, or by incubation at high altitude of sea-level eggs with oxygen supplementation. Prolonged high-altitude residence ancestry confers partial protection against the effects of high-altitude incubation on growth but not on cardiovascular remodeling. It is of obvious interest whether these cardiovascular changes in ovo persist, resolve or amplify in later life.

\section{Acknowledgements}

We thank Dr Diva Bellido, Dr Wilma Tellez, Mr Armando Rodriguez, Mrs Martha Aguilar, Mrs Loyola Riveros, $\mathrm{Mr}$ Wilmar Velasquez and Mr Didi Maquera at IBBA, La Paz; Dr Ginella, Dr Nioshi and Dr Roca at CENETROP, Santa Cruz; and Ms Lilian Kessels at Maastricht University, for their help with these studies. The present study was funded by The British Heart Foundation and The Lister Institute for Preventive Medicine. D.A.G. is a Royal Society-Wolfson Foundation Research Merit Award Holder.

\section{Statement of Interest}

There are no conflicts of interest.

\section{References}

1. Huxley RR, Shiell AW, Law CM. The role of size at birth and postnatal catch-up growth in determining systolic blood pressure: a systematic review of the literature. J Hypertens. 2000; $18,815-831$.

2. Lenfant C. Low birth weight and blood pressure. Metab Clin Exp. 2008; 57, S32-S35.

3. Godfrey KM, Barker DJ. Fetal programming and adult health. Public Health Nutr. 2001; 4, 611-624.

4. Gluckman PD, Hanson MA, Cooper C, Thornburg KL. Effect of in utero and early-life conditions on adult health and disease. $N$ Engl J Med. 2008; 359, 61-73.

5. McMillen IC, Robinson JS. Developmental origins of the metabolic syndrome: prediction, plasticity, and programming. Physiol Rev. 2005; 85, 571-633.

6. Armitage JA, Taylor PD, Poston L. Experimental models of developmental programming: consequences of exposure to an energy rich diet during development. J Physiol. 2005; 565(Pt 1), 3-8.

7. Langley-Evans SC, Bellinger L, McMullen S. Animal models of programming: early life influences on appetite and feeding behaviour. Matern Child Nutr. 2005; 1, 142-148.

8. Zhang L. Prenatal hypoxia and cardiac programming. J Soc Gynecol Investig. 2005; 12, 2-13.

9. Fowden AL, Giussani DA, Forhead AJ. Intrauterine programming of physiological systems: causes and consequences. Physiology (Bethesda). 2006; 21, 29-37.

10. Morrison JL. Sheep models of intrauterine growth restriction: fetal adaptations and consequences. Clin Exp Pharmacol Physiol. 2008; 35, 730-743.

11. Moore LG. Maternal $\mathrm{O}_{2}$ transport and fetal growth in Colorado, Peru and Tibet high-altitude residents. Am J Hum Biol. 1990; 2, 627-637. 
12. Lichty JA, Ting RY, Bruns P, Dyer E. Studies of babies born at high altitude. 1. Relation of altitude to birth weight. Am J Dis Child. 1957; 93, 666-669.

13. McClung J. Effects of High Altitude on Human Birth, 1969. Harvard University Press, Cambridge.

14. Haas JD, Frongillo EF, Stepcik C, Beard J, Hurtado L. Altitude, ethnic and sex differences in birthweight and length in Bolivia. Hum Biol. 1980; 52, 459-477.

15. Moore LG, Niermeyer S, Zamudio S. Human adaptation to high altitude: regional and life-cycle perspectives. Am J Phys Anthropol Suppl. 1998; 27, 25-64.

16. Giussani DA, Phillips PS, Anstee S, Barker DJ. Effects of altitude vs. economic status on birth weight and body shape at birth. Ped Res. 2001; 49, 490-494.

17. Zamudio S, Postigo L, Illsley NP, et al. Maternal oxygen delivery is not related to altitude- and ancestry-associated differences in human fetal growth. J Physiol. 2007; 582(Pt 2), 883-895.

18. Giussani DA, Salinas CE, Villena M, Blanco CE. The role of oxygen in prenatal growth: studies in the chick embryo. J Physiol. 2007; 585(Pt 3), 911-917.

19. West JB. Respiratory Physiology. The Essentials, 7th edn, 2004. Lippincott Williams \& Wilkins, Baltimore.

20. Burton GJ, Palmer ME. Eradicating fetomaternal fluid shift during perfusion fixation of the human placenta. Placenta. 1998; 9, 327-332.

21. Armitage P, Berry G. Further analyses of straight-line data. In Statistical Methods in Medical Research (eds. Armitage P, Berry G, Matthews JNS), 1994; 292-305. Blackwell, Oxford.

22. Clark KE, Durnwald M, Austin JE. A model for studying chronic reduction in uterine blood flow in pregnant sheep. Am J Physiol. 1982; 242, H297-H301.

23. Jensen A, Roman C, Rudolph AM. Effects of reducing uterine blood flow on fetal blood flow distribution and oxygen delivery. J Dev Physiol. 1991; 15, 309-323.

24. Richardson BS, Bocking AD. Metabolic and circulatory adaptations to chronic hypoxia in the fetus. Comp Biochem Physiol A Mol Integr Physiol. 1998; 119, 717-723.

25. Gardner DS, Fletcher AJ, Fowden AL, Giussani DA. A novel method for controlled and reversible long term compression of the umbilical cord in fetal sheep. J Physiol. 2001; 535(Pt 1), 217-229.

26. Murotsuki J, Challis JR, Han VK, Fraher LJ, Gagnon R. Chronic fetal placental embolization and hypoxemia cause hypertension and myocardial hypertrophy in fetal sheep. Am J Physiol. 1997; 272(1 Pt 2), R201-R207.

27. Walker AM, de Preu ND, Horne RS, Berger PJ. Autonomic control of heart rate differs with electrocortical activity and chronic hypoxaemia in fetal lambs. J Dev Physiol. 1990; 14, 43-48.

28. Robinson JS, Kingston EJ, Jones CT, Thorburn GD. Studies on experimental growth retardation in sheep. The effect of removal of endometrial caruncles on fetal size and metabolism. J Dev Physiol. 1979; 1, 379-398.

29. Kitanaka T, Alonso JG, Gilbert RD, Siu BL, Clemons GK, Longo LD. Fetal responses to long-term hypoxemia in sheep. Am J Physiol. 1989; 256, R1348-R1354.

30. Kamitomo M, Longo LD, Gilbert RD. Right and left ventricular function in fetal sheep exposed to long-term high-altitude hypoxemia. Am J Physiol. 1992; 262(2 Pt 2), H399-H405.

31. Thompson LP, Dong Y. Chronic hypoxia decreases endothelial nitric oxide synthase protein expression in fetal guinea pig hearts. J Soc Gynecol Investig. 2005; 12, 388-395.

32. Williams SJ, Hemmings DG, Mitchell JM, McMillen IC, Davidge ST. Effects of maternal hypoxia or nutrient restriction during pregnancy on endothelial function in adult male rat offspring. J Physiol. 2005; 565, 125-135.

33. Xu Y, Williams SJ, O’Brien D, Davidge ST. Hypoxia or nutrient restriction during pregnancy in rats leads to progressive cardiac remodeling and impairs postischemic recovery in adult male offspring. FASEB J. 2006; 20, 1251-1253.

34. Rueda-Clausen CF, Morton JS, Davidge ST. Effects of hypoxiainduced intrauterine growth restriction on cardiopulmonary structure and function during adulthood. Cardiovasc Res. 2009; $81,713-722$.

35. De Grauw TJ, Myers R, Scott WJ. Fetal growth in rats from different levels of hypoxia. Biol Neonate. 1986; 49, 85-89.

36. Ruijtenbeek K, le Noble FAC, Janssen GMJ, et al. Chronic hypoxia stimulates periarterial sympathetic nerve development in chicken embryo. Circulation. 2000; 102, 2892-2897.

37. Rouwet EV, Tintu AN, Schellings MW, et al. Hypoxia induces aortic hypertrophic growth, left ventricular dysfunction, and sympathetic hyperinnervation of peripheral arteries in the chick embryo. Circulation. 2002; 105, 2791-2796.

38. Villamor E, Kessels CG, Ruijtenbeek K, et al. Chronic in ovo hypoxia decreases pulmonary arterial contractile reactivity and induces biventricular cardiac enlargement in the chicken embryo. Am J Physiol Regul Integr Comp Physiol. 2004; 287, R642-R651.

39. Tintu A, Rouwet E, Verlohren S, et al. Hypoxia induces dilated cardiomyopathy in the chick embryo: mechanism, intervention, and long-term consequences. PLoS ONE. 2009; 4, e5155.

40. Anderson PA, Kleinman CS, Lister G, Talner NS. Cardiovascular function during normal fetal and neonatal development and with hypoxic stress. In Fetal and Neonatal Physiology (eds. Polin AR, Fox WW), 1998; pp. 876-889. W.B. Saunders, Philadelphia.

41. Kempf H, Linares C, Corvol P, Gasc JM. Pharmacological inactivation of the endothelin type A receptor in the early chick embryo: a model of mispatterning of the branchial arch derivatives. Development. 1998; 125, 4931-4941.

42. Byrne AM, Bouchier-Hayes DJ, Harmey JH. Angiogenic and cell survival functions of vascular endothelial growth factor (VEGF). J Cell Mol Med. 2005; 9, 777-794.

43. Skilton MR, Evans N, Griffiths KA, Harmer JA, Celermajer DS. Aortic wall thickness in newborns with intrauterine growth restriction. Lancet. 2005; 365, 1484-1486.

44. Koklu E, Kurtoglu S, Akcakus M, et al. Increased aortic intima-media thickness is related to lipid profile in newborns with intrauterine growth restriction. Horm Res. 2006; 65, 269-275.

45. Akira M, Yoshiyuki S. Placental circulation, fetal growth, and stiffness of the abdominal aorta in newborn infants. J Pediatr. 2006; 148, 49-53. 


\section{Chapter IV}

Adrenocortical suppression in

highland chick embryos is restored during incubation at sea

level.

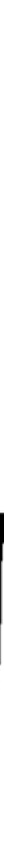

High Alt Med Biol. 2011; 12:79-87 



\title{
Adrenocortical Suppression in Highland Chick Embryos Is Restored during Incubation at Sea Level
}

\author{
Carlos E. Salinas, ${ }^{1}$ Mercedes Villena, ${ }^{1}$ Carlos E. Blanco, ${ }^{2}$ and Dino A. Giussani ${ }^{3}$
}

\begin{abstract}
Salinas, Carlo E., Mercedes Villena, Carlos E. Blanco, and Dino A. Giusssani. Adrenocortical suppression in highland chick embryos is restored during incubation at sea level. High Alt. Med. Biol. 12:79-87, 2011.-By combining the chick embryo model with incubation at high altitude, this study tested the hypothesis that development at high altitude is related to a fetal origin of adrenocortical but not adrenomedullary suppression and that hypoxia is the mechanism underlying the relationship. Fertilized eggs from sea-level or high altitude hens were incubated at sea level or high altitude. Fertilized eggs from sea-level hens were also incubated at altitude with oxygen supplementation. At day 20 of incubation, embryonic blood was taken for measurement of plasma corticotropin, corticosterone, and $\mathrm{Po}_{2}$. Following biometry, the adrenal glands were collected and frozen for measurement of catecholamine content. Development of chick embryos at high altitude led to pronounced adrenocortical blunting, but an increase in adrenal catecholamine content. These effects were similar whether the fertilized eggs were laid by sea-level or high altitude hens. The effects of high altitude on the stress axes were completely prevented by incubation at high altitude with oxygen supplementation. When chick embryos from high altitude hens were incubated at sea level, plasma hormones and adrenal catecholamine content were partially restored toward levels measured in sea-level chick embryos. There was a significant correlation between adrenocortical blunting and elevated adrenal catecholamine content with both asymmetric growth restriction and fetal hypoxia. The data support the hypothesis tested and provide evidence to isolate the direct contribution of developmental hypoxia to alterations in the stress system.
\end{abstract}

Key Words: corticotropin; cortisol; hypoxia; high altitude; chick embryo

\section{Introduction}

I N THE PRENATAL PERIOD, ONE OF THE MOST COMMON forms of stress is fetal hypoxia (Giussani et al., 2001; Thakor and Giussani, 2009). In the late- gestation fetus, hypoxic episodes elicit an integrated defense response that facilitates fetal survival and the protection of hypoxia-sensitive tissues during the period of reduced oxygen availability (Giussani et al., 1994). Increases in fetal plasma catecholamines contribute to the fetal glucogenic (Fowden et al., 1998) and cardiovascular (Giussani et al., 1993) defenses against acute hypoxic stress. Increased fetal plasma concentrations of glucocorticoid amplify the actions of the sympathetic nervous system, contributing also to the fetal metabolic (Fletcher et al., 2000) and cardiovascular (Fletcher et al., 2003) responses to acute stress. However, prolonged elevations in fetal plasma glucocorticoid can reduce fetal growth (Ikegami et al., 1997), trigger preterm birth (Nathanielsz et al., 1988), and program cardiovascular and metabolic defects in later life (Seckl et al., 1999). Therefore, switching the pituitary-adrenocortical axis (HPA) off during prolonged stress is just as important as switching it on during short-term stress (Ducsay, 1998). For instance, in ovine pregnancy, if the fetal exposure to hypoxia lasts from hours to days, dissociation in the plasma corticotropin and cortisol responses occurs, such that glucocorticoid is still maintained high despite plasma corticotropin concentrations returning to basal levels (Murotsuki et al., 1996). However, if the fetal exposure to hypoxia is more prolonged, lasting months, such as during pregnancy at high altitude $(>2500 \mathrm{~m})$, plasma corticotropin remains high while plasma cortisol concentrations return to basal levels (Ducsay, 1998). Blunting of fetal basal adrenocortical output may therefore be an appropriate

\footnotetext{
${ }^{1}$ Instituto Boliviano de Biología de Altura, Facultad de Medicina, Universidad Mayor de San Andrés, La Paz, Bolivia.

${ }^{2}$ Department of Pediatrics, Maastricht University, Maastricht, The Netherlands.

${ }^{3}$ Department of Physiology Development and Neuroscience, University of Cambridge, Downing Street, Cambridge, UK.
} 
homeostatic response to prolonged periods of hypoxic stress to protect sensitive tissues from inappropriate and sustained elevations in plasma glucocorticoid levels during fetal development (Ducsay, 1998).

In human pregnancy, several studies have reported that newborn infants from pregnancies complicated by placental insufficiency are very low birth weight and show adrenocortical suppression (Kajantie et al., 2003; Watterberg et al., 2004). However, because placental insufficiency decreases the delivery of both nutrients and oxygen to the fetus, the extent to which the effects on the developing stress axes are owing to fetal hypoxia or underoxygenation is uncertain. Because most high altitude populations are also impoverished, with a high prevalence of maternal undernutrition (Julian et al., 2009), and because chronic maternal hypoxia in experimental animals also reduces maternal food intake (de Grauw et al., 1986), whether the associated adrenocortical blunting is owing to fetal underoxygenation or undernutrition again remains uncertain.

By combining the chick embryo model with incubation at high altitude, we have previously been able to isolate the direct effects of chronic hypoxia in the control of fetal growth and on fetal cardiovascular development, independent of changes in maternal nutrition or other confounders, such as the maternal and placental physiology (Giussani et al., 2007; Salinas et al., 2010). Since the stress axis is functional before hatching in chicks (Jenkins and Porter, 2004), the present study tested the hypothesis that high altitude is related to fetal adrenocortical but not adrenomedullay suppression. The hypothesis was tested in three ways:

1. By investigating the effects on the stress axes of incubation at high altitude of fertilized eggs laid by sea level-hens

2. By investigating whether alterations in the stress axes induced by development at high altitude could be prevented by incubation at sea level of fertilized eggs laid by high altitude hens

3. By investigating whether alterations in the stress axes induced by development at high altitude could be prevented by incubation at high altitude of sea-level eggs with oxygen supplementation

We were also interested in whether generational high altitude residence altered the effects of chronic hypoxia on the stress system and whether pituitary-adrenal hormone concentration correlated with fetal biometry and/or arterial $\mathrm{Po}_{2}$.

\section{Materials and Methods}

The study was done in Bolivia, in the high altitude city of La Paz $\left(3600 \mathrm{~m}, 494 \mathrm{mmHg}\right.$, approximate ambient dry $\mathrm{Po}_{2}$ $100 \mathrm{mmHg})$ and in the sea-level city of Santa Cruz $(420 \mathrm{~m}$, $760 \mathrm{mmHg}$, approximate ambient dry $\mathrm{Po}_{2} 160 \mathrm{mmHg}$ ). The incubation procedures have been previously published in detail (Giussani et al., 2007; Salinas et al., 2010). In brief, fertilized eggs were obtained from Black Leghorn chickens that had been reared at the sea-level city of Santa Cruz or at the high altitude city of La Paz for at least six generations. Egg storage is commonly practiced in the artificial incubation of domestic birds. If the storage temperature for freshly laid chicken eggs is kept below the physiological zero $\left(25^{\circ}\right.$ to $27^{\circ} \mathrm{C}$ ), dormancy of the embryo can be maintained and fertile eggs can be stored for 1 to 3 weeks. In this study, fertilized eggs from Santa Cruz and La Paz were accumulated, maintained, and transported over 2 to 3 days at $14^{\circ} \mathrm{C}$ to arrest and synchronize development prior to incubation. This permitted incubation at different altitudes to start at day 1 of embryonic age (Giussani et al., 2007). Fertilized eggs from sea-level hens, laid at sea level, were randomly divided and incubated either at sea level (SLSL, $n=35$ ) or high altitude (SLHA, $n=24$ ). Eggs from high altitude hens, laid at high altitude, were randomly divided and incubated either at high altitude (HAHA, $n=36$ ) or sea level (HASL, $n=31$ ). SLHA embryos were also incubated with oxygen supplementation (SLHA $\left.+\mathrm{O}_{2}, n=28\right)$ at rates to maintain sea-level oxygen partial pressures according to Dalton's law (West, 1999).

All incubations (Polyhatch, Brinsea Products Ltd., Sanford, North Somerset, UK) were carried out under conditions to optimize development, with controlled temperature $\left(38^{\circ} \mathrm{C}\right)$ and humidity $(60 \%)$ and appropriate egg rotation. On day 20 of the 21-day incubation period, the egg was weighed, the air cell was exposed, and chorioallantoic venous blood was drawn into a 1-mL syringe for analysis of $\mathrm{PO}_{2}$ (ABL 500, Radiometer, Copenhagen, Denmark), whenever possible in duplicate. At the same time, as much blood as possible (1 to $2 \mathrm{~mL}$ ) was collected from chorioallantoic arterial vessels for subsequent measurement of plasma corticotropin and corticosterone. These samples were collected under sterile conditions into chilled EDTA tubes ( $\mathrm{K}^{+} /$EDTA, LIP Ltd., Shipley, West Yorkshire, UK); they were then centrifuged at $4000 \mathrm{rpm}$ for $4 \mathrm{~min}$ at $4{ }^{\circ} \mathrm{C}$. The plasma obtained was then dispensed into prelabeled tubes, and the samples were stored at $-80^{\circ} \mathrm{C}$ until analysis.

Following euthanasia by spinal transection, the embryo was removed from the eggshell, the adrenal glands were isolated, and embryonic body weight and the combined adrenal weight were recorded. The adrenal glands were snap frozen in liquid nitrogen for subsequent analysis of catecholamine content, because insufficient blood was available from any one embryo for analysis of circulating plasma concentrations of catecholamines.

Measurements for plasma corticotropin, corticosterone, and adrenal catecholamine content were performed in a subset of animals for each group within 2 months of sample collection, as previously described in detail, (Gardner et al., 2001; Fletcher et al., 2006). Plasma corticotropin and corticosterone concentrations were determined by radioimmunoassay (RIA) using commercially available kits (corticotropin: DiaSorin Inc., Stillwater, Minnesota, USA; corticosterone: ICN Biomedicals, Irvine, CA, USA). For corticotropin, the lower limit of detection for the assay was between 10 and $25 \mathrm{pg} /$ $\mathrm{mL}^{-1}$. The intraassay coefficients of variation for two plasma pools (37 and $150 \mathrm{pg} / \mathrm{mL}^{-1}$ ) were $3.6 \%$ and $4.1 \%$, respectively. The interassay coefficient of variation was $8.4 \%$. For corticosterone, the assay sensitivity was $25 \mathrm{pg} / \mathrm{mL}^{-1}$, and the intra- and interassay coefficients of variation were $5.8 \%$ and $7.5 \%$, respectively. Noradrenaline and adrenaline concentrations were measured in both adrenal glands per chick embryo by high-pressure liquid chromatography (HPLC) using electrochemical detection. The samples were prepared by absorption of $250 \mu \mathrm{L}$ onto acid-washed alumina, and $20 \mu \mathrm{L}$ aliquots of the $100-\mu \mathrm{L}$ perchloric acid elutes was injected onto the column. Dihydroxybenzylamine was added as the internal standard to each sample before absorption. Recovery ranged from $63 \%$ to $97 \%$, and all catecholamine values were corrected for their respective recovery. The interassay coeffi- 
cients of variation for adrenaline and noradrenaline were $7.3 \%$ and $6.2 \%$, respectively. Measurements were made in duplicate on two dilutions of each purified sample, and the data were expressed as $\mu \mathrm{g} / \mathrm{mg}^{-1}$ for tissue content.

All procedures were approved by the local ethics committee of the Bolivian Institute for High Altitude Biology (Consejo Técnico, IBBA, Universidad Mayor de San Andrés, La Paz, Bolivia). Values for $\mathrm{PO}_{2}$, embryonic and adrenal weights, and endocrine variables are expressed as mean \pm SEM. Comparisons between groups were assessed statistically using oneway ANOVA with the Student Newman-Keuls post hoc test (Sigma-Stat, SPSS Inc., Chicago, IL, USA). The relationship between parallel measurements of plasma concentrations of corticotropin and corticosterone in all individual chick embryos was assessed using the Pearson product moment correlation. A comparison between the slopes and intercepts of regression lines was conducted according to Armitage and Berry (1994). For all comparisons, statistical significance was accepted when $p<0.05$.

\section{Results}

\section{Oxygenation and biometry}

Embryonic systemic hypoxia and growth restriction occurred during incubation at high altitude (SLHA and HAHA) (Table 1). When weight was expressed as a percentage of the initial egg mass, embryos from high altitude hens (HAHA) relative to those from sea-level hens (SLHA) showed a diminished reduction in growth during high altitude incubation. Further, when this group was incubated at sea level (HASL), the relative body weight was greater than for any other group. The absolute combined adrenal weight was also reduced during incubation at high altitude (SLHA and HAHA); however, this effect no longer occurred when the combined adrenal weight was expressed relative to the embryonic body weight. Reductions in body and adrenal weights no longer occurred during incubation at high altitude with oxygen supplementation (SLHA $+\mathrm{O}_{2}$ ).

\section{Plasma corticotropin and corticosterone}

Although plasma concentrations of corticotropin were significantly elevated, plasma concentrations of corticosterone were significantly depressed in embryos incubated at high altitude (SLHA and HAHA) relative to sea-level embryos (SLSL; Fig. 1). The magnitudes of the increment in plasma corticotropin and of the decrement in plasma corticosterone following incubation at high altitude were similar in embryos from hens native to sea level (SLHA) or to high altitude (HAHA). Plasma corticotropin in high altitude embryos incubated at sea level (HASL) was no longer different from sea-level embryos (SLSL). Plasma corticosterone concentrations in these embryos (HASL) were significantly greater than in SLHA and HAHA embryos, but still significantly depressed relative to SLSL embryos. Incubation of sealevel embryos at high altitude with oxygen supplementation (SLHA $+\mathrm{O}_{2}$ ) prevented the high altitude-induced increase in plasma corticotropin and the high altitude-induced decrease in plasma corticosterone.

Correlation analysis, using the Pearson product moment test of paired plasma corticotropin and corticosterone values for all individual chick embryos (Fig. 2), revealed significant relationships for sea-level embryos incubated at sea level (SLSL, $r=0.9, n=10, p<0.001$ ) and for sea-level embryos incubated at high altitude with oxygen supplementation (SLHA $+\mathrm{O}_{2}, r=0.9, n=9, p<0.003$ ). However, no significant relationship between plasma corticotropin and corticosterone was found in sea-level embryos incubated at high altitude (SLHA, $r=0.7, n=7, p=0.07$ ), in high altitude embryos incubated at high altitude (HAHA, $r=0.1, n=7, p=0.90$ ), or in high altitude embryos incubated at sea level (HASL, $r=0.1$, $n=9, p=0.88$ ).

A comparison of slopes of the linear regressions (Fig. 2) also revealed that the slopes of sea-level chick embryos incubated at high altitude (SLHA, $y=0.0101 x+2.4$ ), of high altitude embryos incubated at high altitude (HAHA, $y=0.0007 x+$ 5.3), and of high altitude embryos incubated at sea level (HASL, $y=0.0017 x+10.5)$ were significantly depressed $(p<0.05)$ relative to sea-level embryos incubated at sea level (SLSL, $y=0.1103 x-3.4$ ) or sea-level embryos incubated at high altitude with oxygen supplementation (SLHA $+\mathrm{O}_{2}$, $y=0.0838 x+11.0)$.

\section{Adrenal catecholamine content}

Adrenal concentrations of noradrenaline and adrenaline were significantly elevated in embryos incubated at high altitude (SLHA and HAHA) relative to sea-level embryos (SLSL; Fig. 3). The magnitudes of these increments in adrenal catecholamine content following incubation at high altitude were similar in embryos from hens native to both sea level (SLHA) and high altitude (HAHA). Adrenal catecholamine content in high altitude embryos incubated at sea level (HASL) were significantly depressed relative to SLHA and HAHA embryos, but still significantly elevated relative to SLSL embryos. Incubation of sea-level embryos at high altitude with oxygen

Table 1. Oxygenation and Biometry in the Chick Embryo

\begin{tabular}{lcccc}
\hline & $\mathrm{PO}_{2}(\mathrm{mmHg})$ & Embryonic body weight (\%) & Adrenal weight (mg) & Relative adrenal weight (\%) \\
\hline SLSL & $57 \pm 3(12)^{\mathrm{a}}$ & $41 \pm 1(35)^{\mathrm{a}}$ & $5.5 \pm 0.3(35)^{\mathrm{a}}$ & $0.020 \pm 0.001(35)$ \\
SLHA & $34 \pm 2(14)^{\mathrm{b}}$ & $21 \pm 1(24)^{\mathrm{b}}$ & $3.6 \pm 0.3(24)^{\mathrm{b}}$ & $0.025 \pm 0.002(24)$ \\
$\mathrm{HAHA}$ & $35 \pm 1(13)^{\mathrm{b}}$ & $29 \pm 1(36)^{\mathrm{c}}$ & $3.7 \pm 0.3(36)^{\mathrm{b}}$ & $0.024 \pm 0.002(36)$ \\
$\mathrm{HASL}$ & $59 \pm 5(9)^{\mathrm{a}}$ & $54 \pm 2(31)^{\mathrm{d}}$ & $5.2 \pm 0.3(31)^{\mathrm{a}}$ & $0.021 \pm 0.002(31)$ \\
SLHA $+\mathrm{O}_{2}$ & $60 \pm 4(11)^{\mathrm{a}}$ & $46 \pm 1(28)^{\mathrm{a}}$ & $5.9 \pm 0.4(28)^{\mathrm{a}}$ & $0.020 \pm 0.002(28)$ \\
\hline
\end{tabular}

Values are mean \pm SEM for the partial pressure of oxygen in chorioallantoic venous blood, the embryonic weight expressed as a percentage of the initial egg mass, and the absolute combined adrenal weight and the combined adrenal weight expressed as a percentage of the embryonic body weight. Groups are sea-level chick embryos incubated either at sea level (SLSL) or at high altitude (SLHA), high altitude embryos incubated at high altitude (HAHA) or at sea level (HASL), and sea-level chick embryos incubated at high altitude with oxygen supplementation (SLHA $+\mathrm{O}_{2}$ ). Numbers in brackets refer to $n$. Values within columns that have different letters as superscripts are significantly different from each other (one-way ANOVA with Student Newman-Keuls test; $p<0.05$ ). 

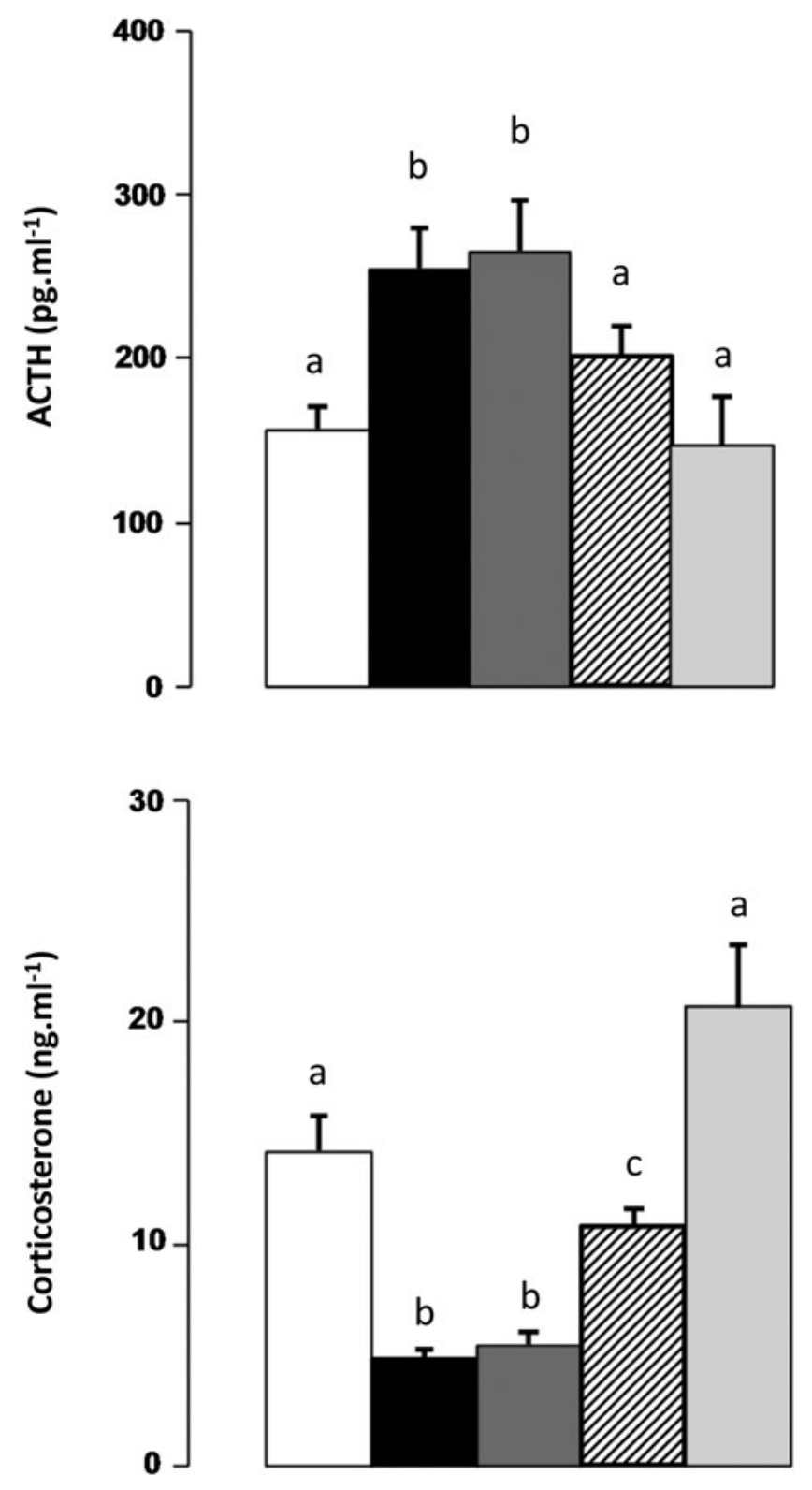

FIG. 1. Plasma corticotropin and corticosterone in the chick embryo. Values are mean \pm SEM for the plasma concentrations of corticotropin and corticosterone in chorioallantoic arterial blood at day 20 of the incubation period. Groups are sea-level chick embryos incubated either at sea level (SLSL, $n=9, \square$ ) or at high altitude (SLHA, $n=7, \mathbf{\square})$, high altitude embryos incubated at high altitude (HAHA, $n=9$, $\square$ ) or at sea level (HASL, $n=7,(2)$, and sea-level chick embryos incubated at high altitude with oxygen supplementation (SLHA $\pm \mathrm{O}_{2}$, $n=10, \quad)$. Values within columns that have different letters as superscripts are significantly different from each other (oneway ANOVA with Student Newman-Keuls test, $p<0.05$ ).

supplementation (SLHA $+\mathrm{O}_{2}$ ) prevented the high altitudeinduced increase in adrenal catecholamine content.

\section{Relation between adrenal function and embryonic size or $\mathrm{PO}_{2}$}

Correlation analysis revealed that the plasma corticosterone-corticotropin ratio was positively related to the embryonic body weight and to $\mathrm{PO}_{2}$, but negatively related to the
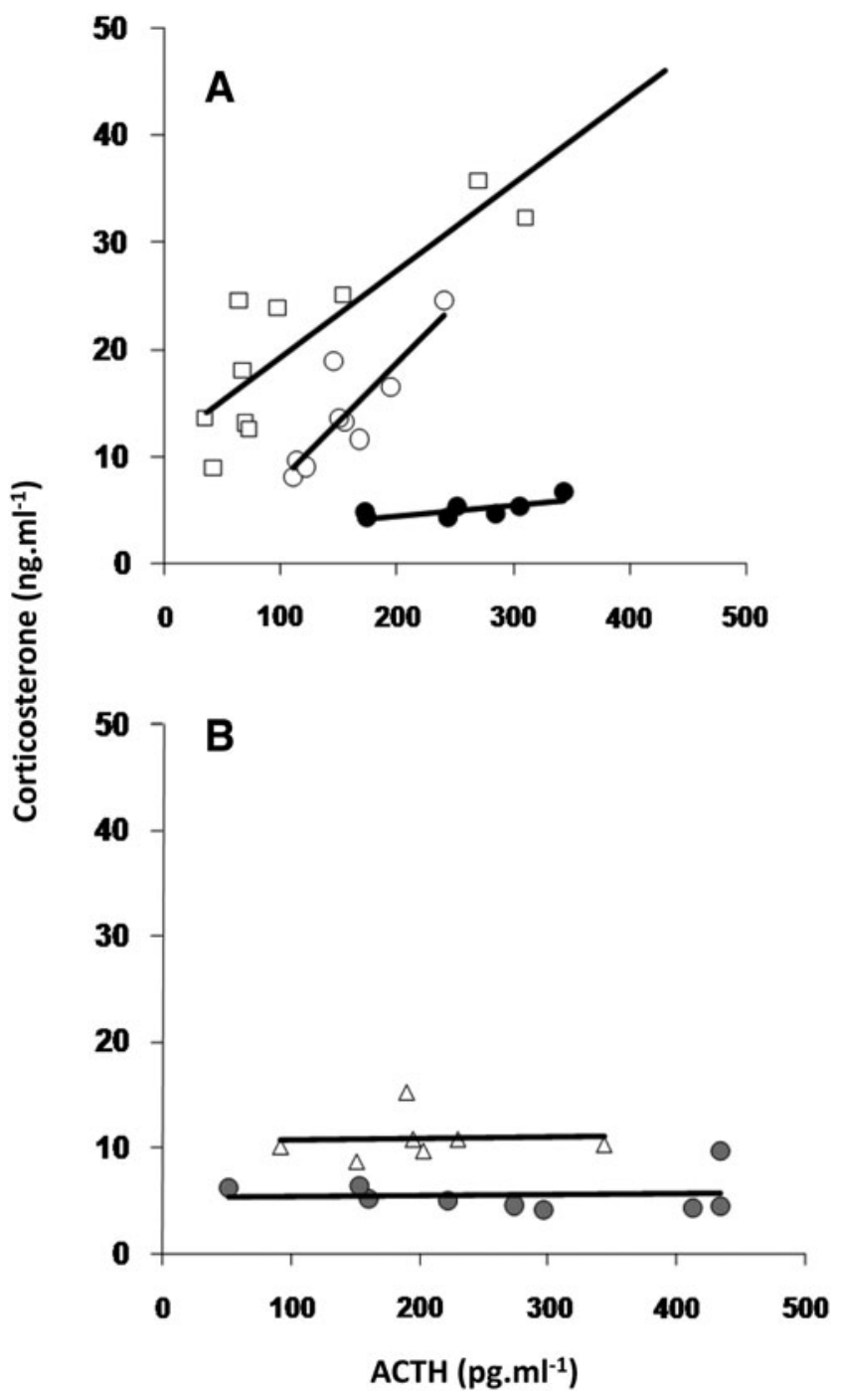

FIG. 2. Relation between plasma corticotropin and corticosterone in the chick embryo.Values are the paired plasma corticotropin and corticosterone concentrations in chorioallantoic blood at day 20 of the incubation period for all individual samples in chick embryos. (A) Sea-level chick embryos incubated either at sea level (SLSL, $n=9, \bigcirc$ ), or at high altitude (SLHA, $n=7 \bullet$ ), or at high altitude with oxygen supplementation (SLHA $\pm \mathrm{O}_{2}, n=10, \square$ ). (B) High altitude embryos incubated either at high altitude (HAHA, $n=9$, O) or at sea level (HASL, $n=7, \triangle$ ).

head diameter-body weight ratio in all groups, independent of treatment (Fig. 4). Conversely, the adrenal catecholamine content was negatively related to embryonic body weight and to $\mathrm{Po}_{2}$, but positively related to the head diameterbody weight ratio in all groups, independent of treatment (Fig. 4).

\section{Discussion}

The data show that the development of chick embryos at high altitude leads to pronounced adrenocortical blunting, but an increase in adrenal catecholamine content, by the end of the incubation period. These effects of high altitude incubation are similar whether the fertilized eggs were laid by sealevel or high altitude hens. The effects of high altitude on the stress axes are completely prevented by incubation at high 

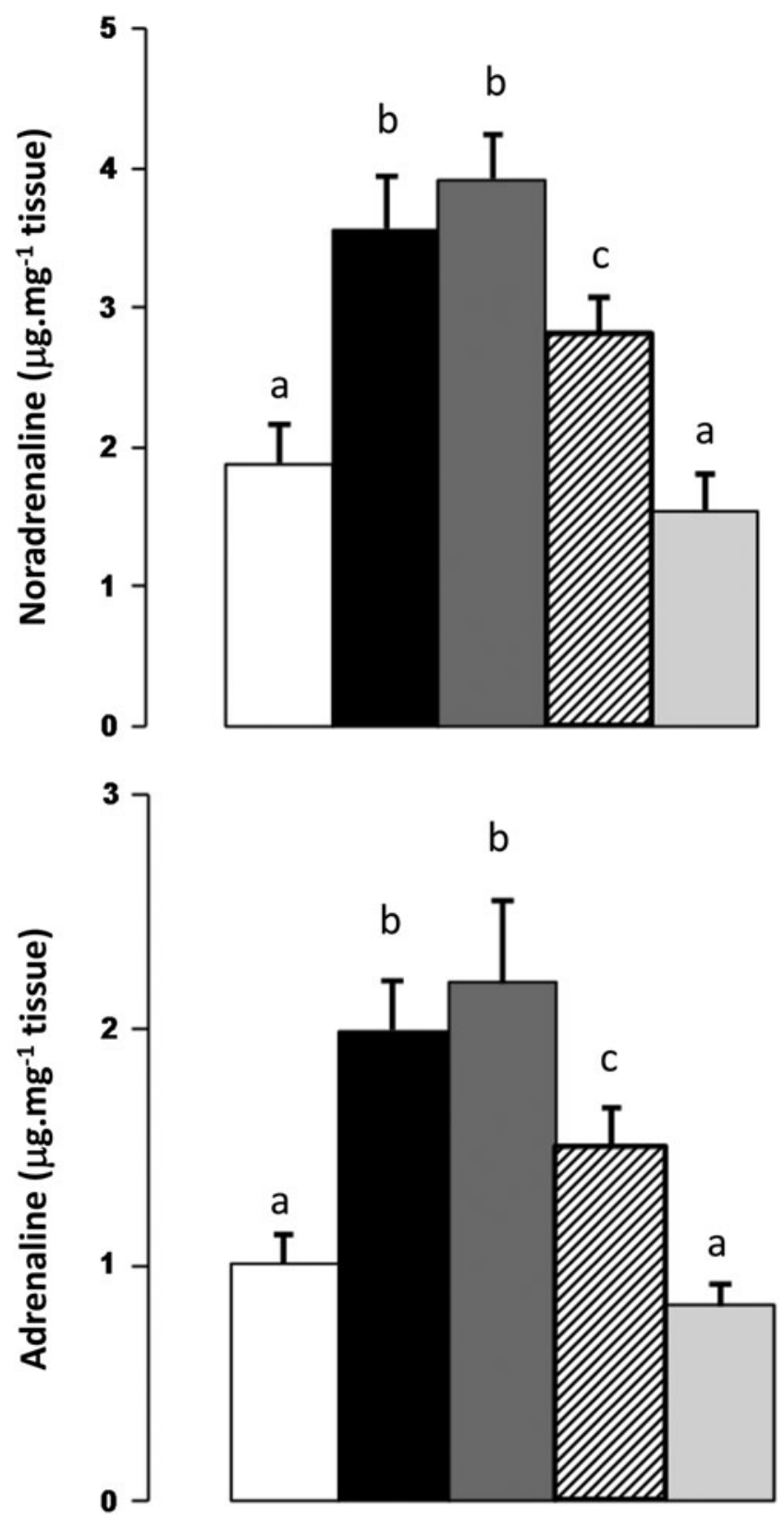

FIG. 3. Adrenal catecholamines in the chick embryo. Values are mean \pm SEM for the content noradrenaline and adrenaline expressed per milligram of tissue at day 20 of the incubation period. Groups are sea-level chick embryos incubated either at sea level (SLSL, $n=10, \square$ ) or at high altitude (SLHA, $n=10, \mathbf{\square}$ ), high altitude embryos incubated at high altitude (HAHA, $n=10$, $\square$ ) or at sea level (HASL, $n=9$, \%), and sea-level chick embryos incubated at high altitude with oxygen supplementation (SLHA $\pm \mathrm{O}_{2}, n=10, \cdots$ ). Values within columns that have different letters as superscripts are significantly different from each other (one-way ANOVA with Student Newman-Keuls test, $p<0.05$ ).

altitude with oxygen supplementation. When chick embryos from high altitude hens are incubated at sea level, plasma corticosterone and adrenal catecholamines by the end of incubation are partially restored toward levels measured in sealevel chick embryos. Adrenal cortical blunting and elevated catecholamine concentrations were significantly related to both growth restriction and fetal hypoxia.
Basal plasma concentrations of corticosterone in embryos from sea-level hens incubated at sea level were within the range of those reported by others for chick embryos at this stage of incubation or at hatching (Marie, 1981; Scott et al., 1981). Although several studies have reported that the adrenal cortex of the chick embryo is functionally responsive to corticotropin by the end of the incubation period (Woods et al., 1971; Wise and Frye, 1973), to our knowledge, this is the first report of circulating levels of corticotropin in the chick embryo during development at both at sea level and high altitude. One previous study has published the adrenaline content of the chick embryo adrenal gland during development (Wassermann and Bernard, 1970). In the present study, the levels of adrenaline content in the adrenal gland of embryos from sea-level hens incubated at sea level were similar to those reported by Wasserman and Bernard (1970) for chick embryos at this stage of incubation.

The physiology underlying the suppression of fetal adrenocortical function during development at high altitude has been studied extensively in a series of elegant contributions by Ducsay and Myers. Using the long-term hypoxemic (LTH) ovine model, whereby pregnant ewes are maintained at $3820 \mathrm{~m}$ above sea level from day 30 of gestation (term is $\sim 150$ days), they reported that the ability of the late-gestation sheep fetus to respond to a corticotropin challenge is markedly suppressed (Harvey et al., 1993). In addition, LTH sheep fetuses have significantly enhanced anterior pituitary processing of proopiomelanocortin (POMC) to corticotropin (Myers et al., 2005a) and an enhanced pituitary responsiveness to arginine vasopressin (Ducsay et al., 2009), both of which result in greater basal corticotropin concentrations, but in similar concentrations of cortisol (Ducsay, 1998). These findings are consistent with blunting of fetal basal adrenocortical output, which may be an appropriate adaptive fetal response to prolonged stress, to protect itself against the deleterious effects on fetal growth and the development of inappropriate and sustained elevations in plasma glucocorticoid levels (Ducsay, 1998). Reduced expression of adrenal corticotropin receptor and key steroidogenic enzymes (Myers et al., 2005b), as well as increased NO-mediated inhibition of steroidogenesis (Monau et al., 2009), may contribute to the physiology underlying adrenocortical blunting in the high altitude fetus. The data in the present article showed that adrenocortical blunting could be induced in chick embryos incubated at high altitude. Indeed, incubation at high altitude of fertilized eggs from sea-level hens produced severely growth restricted embryos with basal plasma corticosterone concentrations substantially lower than sea-level embryos. Adrenocortical blunting could be prevented by high altitude incubation with oxygen supplementation, and it could be reversed by incubating chick embryos from high altitude hens at sea level. Therefore, the present data extend the findings of Ducsay and Myers to confirm, by using a three-prong approach, that it is the direct effect of hypoxia during development at high altitude, rather than maternal or placental factors and/or hypobaria, that is responsible for adrenocortical blunting in the fetus. Of interest, our data also show that there is a strong positive relationship between adrenocortical blunting and intrauterine growth retardation (IUGR) and a strong negative relationship between adrenocortical blunting and arterial $\mathrm{Po}_{2}$ in the chick embryo. These significant relationships further support the concepts that development under chronic hypoxia promotes IUGR (Giussani et al., 2007; 

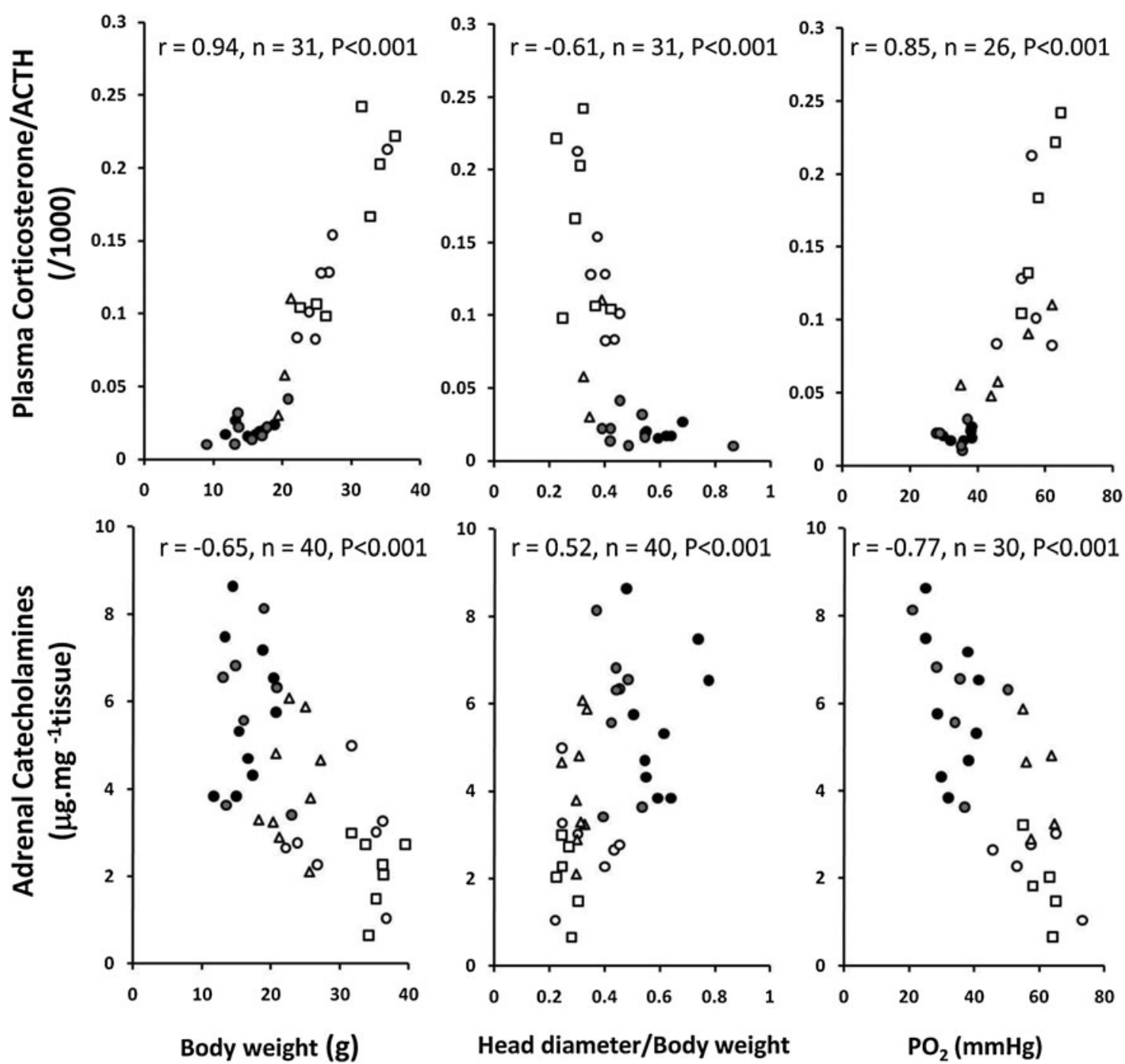

FIG. 4. Relationship between adrenal function and embryonic size or $\mathrm{Po}_{2}$. Body weight, the head diameter to body weight ratio, and chorioallantoic venous $\mathrm{PO}_{2}$ at the end of the incubation period were related to the ratio of corticosteronecorticotropin in plasma and the adrenal combined catecholamine content in all embryos independent of treatment. $r$, Pearson product moment correlation coefficient; $n$, number of observations. SLSL (O), SLHA $(\bullet)$, HAHA (O), HASL $(\triangle)$, and $\mathrm{SLHA} \pm \mathrm{O}_{2}(\square)$.

Copeland and Dzialowski, 2009; Tintu et al., 2009) and that hypoxia-sensitive genes are involved in adrenocortical blunting.

Although blunting of basal adrenocortical output during long-term hypoxic development may protect the fetus against the deleterious effects of sustained elevations in glucocorticoid, it also renders the mammalian fetus susceptible to an inappropriate adrenocortical defense to a secondary acute stressor, such as the intrapartum hypoxia at the end of pregnancy. Our group (Riquelme et al., 1998; Riquelme et al., 2002) and Ducsay and Myers (Adachi et al., 2004; Imamura et al., 2004) have hypothesized that possible mechanisms exist to override suppression of fetal adrenocortical function during superimposed stress during development at high altitude. One strategy may be to increase acutely the gain of neural influences on adrenocortical function, which are triggered by a carotid chemoreflex (Riquelme et al., 1998; Riquelme et al, 2002) and mediated by splanchnic innervation to the adrenal gland (Myers et al., 1990). Compared to sheep, such neural influences on adrenocortical ouput during acute stress in the fetus are sensitized in the llama (Riquelme et al., 1998; Riquelme, 2002), a species adapted to the chronic hypoxia of life at high altitude for generational times (Giussani et al., 1999). Ducsay and Myers have recently reported (Vargas et al., 2010) that the enhanced plasma cortisol response to a second stressor in the LTH sheep fetus may involve enhanced adrenocortical intracellular signaling downstream from cAMPdependent protein kinase A (PKA). Data in the present article also show that, when chick embryos from hens native to high altitude are incubated at sea level, plasma corticosterone by the end of incubation is partially restored toward the concentrations measured in sea-level chick embryos. However, the mechanism promoting this increase appears independent of corticotropin, because the relation between corticosterone and corticotropin in this group is still blunted relative to sealevel chick embryos. It is possible that the mechanisms employed by the highland chick embryo to restore adrenocortical output during incubation at sea level are similar to those 
employed by the mammalian fetus to unleash an adrenocortical response of appropriate magnitude in response to a second stressor, despite basal adrenocortical blunting.

In contrast to the present study, Hassanzadeh and colleagues (2004) reported significantly higher plasma corticosterone concentrations in chick embryos following highland incubation compared to incubation at sea level. However, that study has several important differences. They incubated chick embryos at 2000-m altitude relative to $\sim 4000$ - $\mathrm{m}$ altitude as in the present study. Also, their blood samples were obtained by cardiac puncture, rather than by chorioallantoic arterial blood sampling. It is likely that the difference in the effect of high altitude incubation on plasma corticosterone in the chick embryo between their and our studies is owing to the actual level of fetal hypoxia experienced. Blood sampling by cardiac puncture is stressful. Therefore, the significantly higher plasma corticosterone concentrations measured in highland chick embryos in the study of Hassanzadeh and colleagues (2004) may represent a greater capacity of the adrenal cortex to respond to acute stress, rather than basal concentrations during chronic exposure to high altitude. Therefore, the finding is in keeping with the hypothesis that possible mechanisms exist to override suppression of fetal adrenocortical function during superimposed stress following development at high altitude (Riquelme et al., 1998; Riquelme et al., 2002; Adachi et al., 2004; Imamura et al., 2004).

By late gestation in the developing ovine fetus, stress activates the sympathetic nervous system, eliciting the release of both noradrenaline and adrenaline. These catecholamines play an essential role in regulating fetal cardiovascular and metabolic responses to a wide variety of stresses and are fundamental in the adaptation of the neonate to the environment after birth (Giussani et al., 1993; Fowden et al., 1998; Olver et al., 2004). Several investigators have reported that fetal exposure to chronic hypoxia leads to upregulation of the sympathoadrenomedullary system. For instance, chronically hypoxemic fetal sheep (Gardner et al., 2002) and fetal llamas (Llanos et al., 2003) have higher resting plasma concentrations of noradrenaline than normoxic fetal sheep at equivalent stages of gestation. Studies by Ruijtenbeck and colleagues (2000) have reported that development of chick embryos under chronic isobaric hypoxia from 0.3 to 0.9 of the incubation period leads to elevations in the vascular noradrenaline content of peripheral circulations. Of interest, Camm and colleagues (2004) reported that the increase in catecholamine as a consequence of chronic hypoxia during development induced impaired memory in chicken. It has also been reported that both llama fetuses (Giussani et al., 1999) and chronically hypoxemic, growth-retarded fetal sheep (Creasy et al., 1973) have a greater dependence on $\alpha$-adrenergic mechanisms to survive episodes of acute hypoxic stress than do control fetal sheep. Data in the present study show that in the chick embryo (1) adrenal catecholamine content is enhanced by development at high altitude, (2) this effect could be prevented by high altitude incubation with oxygen supplementation and could be reversed by incubating chick embryos from high altitude hens at sea level, and (3) there is a strong negative relationship between arterial $\mathrm{Po}_{2}$ and adrenal catecholamine content and a strong positive relationship between adrenal catecholamine content and IUGR. Therefore, the present data also extend previous findings to confirm that it is the direct effect of reductions in oxygenation during chronic hypoxic development that leads to upregulation of the sympathoa- drenomedullary system, of the type which is associated with IUGR.

In conclusion, the combined data in the present study provide strong evidence to support the hypothesis tested. High altitude is related to fetal adrenocortical but not to adrenomedullary suppression, and hypoxia is the mechanism underlying the relationship. Blunting of fetal basal adrenal cortical but not medullary function may be an appropriate homeostatic response to prolonged periods of hypoxia to protect sensitive tissues from sustained elevations of plasma cortisol levels while maintaining appropriate glucogenic capacity during fetal development. The biological trade-off may yield newborns with adrenocortical suppression.

\section{Acknowledgments}

We thank Dr. Diva Bellido, Dr. Wilma Tellez, Mr. Armando Rodriguez, Mrs. Martha Aguilar, Mrs. Loyola Riveros, Mr. Wilmar Velasquez, and Mr. Didi Maquera at IBBA, La Paz; Dr. Ginella, Dr. Nioshi, and Dr. Roca at CENETROP, Santa Cruz; and Mr. Malcolm Bloomfield at the University of Cambridge for their help with these studies.

This work was supported by the Lister Institute for Preventive Medicine, the British Heart Foundation, and the BBSRC. Dino A. Giussani is a current holder of the Royal Society's Wolfson Research Merit award.

\section{Disclosures}

The authors have no conflicts of interest or financial ties to disclose.

\section{References}

Adachi K., Umezak H., Kaushal K.M., and Ducsay, C.A. (2004). Long-term hypoxia alters ovine fetal endocrine and physiological responses to hypotension. Am. J. Physiol. Regul. Integr. Comp. Physiol. 287:R209-R217.

Armitage P., and Berry G. (1994). Further analyses of straightline data. In: Statistical Methods in Medical Research. Blackwells, Oxford, UK; 292-305.

Camm E.J., Hansell J.A., Kane A.D., Herrera E.A., Lewis C., Wong S., Morrell N.W., and Giussani D.A. (2010). Partial contributions of developmental hypoxia and undernutrition to prenatal alterations in somatic growth and cardiovascular structure and function. Am. J. Obstet. Gynecol. 203:495.e, 24-34.

Camm, E.J., Harding, R., Lambert, G.W., Gibbs, M.E. (2004). The role of catecholamines in memory impairment in chicks following reduced gas exchange in ovo. Neuroscience 128:545553.

Copeland J., and Dzialowski E.M. (2009). Effects of hypoxic and hyperoxic incubation on the reactivity of the chicken embryo (Gallus gallus) ductus arteriosi in response to catecholamines and oxygen. Exp. Physiol. 94(1):152-161.

Creasy R.K., Deswieft M., Kahanpaa K.V., Young W.P., and Rudolph A.M. (1973). Pathophysiological changes in the foetal lamb with growth retardation. In: Foetal and Neonatal Physiology. Proceedings of the Barcroft Centenary Symposium. R. S. Comline, K. W. Cross, G. S. Dawes, and P. W. Nathanielsz, eds. Cambridge University Press, London; pp. 398-402.

de Grauw T.J., Myers R.E., and Scott W.J. (1986). Fetal growth retardation in rats from different levels of hypoxia. Biol. Neonate. 49:85-89.

Ducsay C.A. (1998). Fetal and maternal adaptations to chronic hypoxia: prevention of premature labor in response to chronic 
stress. Comp. Biochem. Physiol. A. Mol. Integr. Physiol. 119:675-681.

Ducsay C.A., Mlynarczyk M., Kaushal K.M., Hyatt K., Hanson K., and Myers D.A. (2009). Long-term hypoxia enhances corticotropin response to arginine vasopressin but not corticotropin-releasing hormone in the near-term ovine fetus. Am. J. Physiol. Regul. Integr. Comp. Physiol. 297:R892-R899.

Fletcher A.J., Gardner D.S., Edwards C.M., Fowden A.L., and Giussani D.A. (2003). Cardiovascular and endocrine responses to acute hypoxaemia during and following dexamethasone infusion in the ovine fetus. J. Physiol. 549(Pt. 1): 271-287.

Fletcher A.J.W., Gardner D.S., Edwards C.M.B., Fowden A.L., and Giussani D.A. (2006). Development of the ovine fetal cardiovascular defense to hypoxemia towards full term. Am. J. Physiol. Heart. Circ. Physiol. 291:H3023-H3034.

Fletcher A.J., Goodfellow M.R., Forhead A.J., Gardner D.S., McGarrigle H.H.G., Fowden A.L., and Giussani D.A. (2000). Low doses of dexamethasone suppress pituitary-adrenal function but augment the glycemic response to acute hypoxemia in fetal sheep during late gestation. Pediatr. Res. 47:18.

Fowden A.L., Mundy L., and Silver M. (1998). Developmental regulation of glucogenesis in the sheep fetus during late gestation. J. Physiol. 508:937-947.

Gardner D.S., Fletcher A.J., Bloomfield M.R., Fowden A.L., and Giussani D.A. (2002). Effects of prevailing hypoxaemia, acidaemia or hypoglycaemia upon the cardiovascular, endocrine and metabolic responses to acute hypoxaemia in the ovine fetus. J. Physiol. 540:351-366.

Gardner D.S., Fletcher A.J.W., Fowden A.L., and Giussani D.A. (2001). Plasma adrenocorticotropin and cortisol concentrations during acute hypoxemia after a reversible period of adverse intrauterine conditions in the ovine fetus during late gestation. Endocrinology. 142:589-598.

Giussani D.A., Gardner D.S., Cox D.T., and Fletcher A.J. (2001). Purinergic contribution to circulatory, metabolic, and adrenergic responses to acute hypoxemia in fetal sheep. Am. J. Physiol. Regul. Integr. Comp. Physiol. 2280(3):R678-R685.

Giussani D.A., Riquelme R.A., Sanhueza E.M., Hanson M.A., Blanco C.E., and Llanos A.J. (1999). Adrenergic and vasopressinergic contributions to the cardiovascular response to acute hypoxaemia in the llama fetus. J. Physiol. 515:233-241.

Giussani D.A., Salinas C.E., Villena M., and Blanco C.E. (2007). The role of oxygen in prenatal growth: studies in the chick embryo. J. Physiol. 585:911-917.

Giussani D.A., Spencer J., and Hanson M.A. (1994). Fetal cardiosvascular reflex responses to hypoxaemia. Fetal Maternal Med. Rev. 6:17-37.

Giussani D.A., Spencer J.A., Moore P.J., Bennet L., and Hanson M.A. (1993). Afferent and efferent components of the cardiovascular reflex responses to acute hypoxia in term fetal sheep. J. Physiol. 461:431-449.

Harvey L.M., Gilbert R.D., Longo L.D., and Ducsay C.A. (1993). Changes in ovine fetal adrenocortical responsiveness after long-term hypoxemia. Am. J. Physiol. (Endocrinol. Metab.) 264:E741-E747.

Hassanzadeh M., Fard M.H., Buyse J., Bruggeman V., and Decuypere E. (2004). Effect of chronic hypoxia during embryonic development on physiological functioning and on hatching and post-hatching parameters related to ascites syndrome in broiler chickens. Avian Pathol. 33(6):558-564.

Ikegam M., Jobe A.H., Newnham J., Polk D.H., Willet K.E., and Sly P. (1997). Repetitive prenatal glucocorticoids improve lung function and decrease growth in preterm lambs. Am. J. Resp. Crit. Care Med. 156:178-184.
Imamura T.U.H., Kaushal K.M., and Ducsay C.A. (2004). Longterm hypoxia alters endocrine and physiologic responses to umbilical cord occlusion in the ovine fetus. J. Soc. Gynecol. Investig. 11:131-140.

Jenkins S.A., and Porter T.E. (2004). Ontogeny of the hypothalamo-pituitary-adrenocortical axis in the chicken embryo: a review. Domestic Animal Endocrinol. 26:267-275.

Julian C.G., Wilson M.J., and Moore L.G. (2009). Evolutionary adaptation to high altitude: a view from in utero. Am. J. Human Biol. 21:614-622.

Kajantie E., Dunkel L., Turpeinen U., Stenman U.-H., Wood P.J., Nuutila M., and Andersson S. (2003). Placental 11 betahydroxysteroid dehydrogenase- 2 and fetal cortisol/cortisone shuttle in small preterm infants. J. Clin. Endocrinol. Metab. 88:493-500.

Llanos A.J, Riquelme R.A., Sanhueza E.M., Hanson M.A., Blanco C.E., Parer J.T., Herrera E.A., Pulgar V.M., Reyes R.V., Cabello G., and Giussani D.A. (2003). The fetal llama versus the fetal sheep: different strategies to withstand hypoxia. High Alt. Med. Biol. 4:193-202.

Marie C. (1981). Ontogenesis of the adrenal glucocorticoids and of the target function of the enzymatic tyrosine transaminase activity in the chick embryo. J. Endocrinol. 90:193-200.

Monau T.R., Vargas V.E., King N., Yellon S.M., Myers D.A., and Ducsay C.A. (2009). Long-term hypoxia increases endothelial nitric oxide synthase expression in the ovine fetal adrenal. Reprod. Sci. 16:865-874.

Murotsuki J., Gagnon R., Matthews S.G., and Challis J.R. (1996). Effects of long-term hypoxemia on pituitary-adrenal function in fetal sheep. Am. J. Physiol. Endocrinol. Metab. 271:E678E685.

Myers D.A., Bell P.A., Hyatt K., Mlynarczyk M., and Ducsay C.A. (2005a). Long-term hypoxia enhances proopiomelanocortin processing in the near-term ovine fetus. Am. J. Physiol. Regul. Integr. Comp. Physiol. 288:R1178-R1184.

Myers D.A., Hyatt K., Mlynarczyk M., Bird I.M., and Ducsay C.A. (2005b). Long-term hypoxia represses the expression of key genes regulating cortisol biosynthesis in the near-term ovine fetus. Am. J. Physiol. Regul. Integr. Comp. Physiol. 289:R1707-R1714.

Myers D.A., Robertshaw D., and Nathanielsz P.W. (1990). Effect of bilateral splanchnic nerve section on adrenal function in the ovine fetus. Endocrinology. 127(5):2328-2335.

Nathanielsz P.W., Buster J.E., Jenkin G., Jorgensen G., and Thorburn G.D. (1988). Induction of premature delivery in sheep following infusion of cortisol to the fetus: the effect of maternal progestagen treatment on the c21-steroid-17alphahydroxylase, c-17, 20 lyase, and aromatase pathways. J. Developmental Physiol. 10:257-270.

Olver R.E., Walters D.V., and M, Wilson, S. (2004). Developmental regulation of lung liquid transport. Annu. Rev. Physiol. 66:77-101.

Riquelme R.A., Llanos J.A., McGarrigle H.H.G., Sanhueza E.M., Hanson M.A., and Giussani D.A. (1998). Chemoreflex contribution to adrenocortical function during acute hypoxemia in the llama fetus at 0.6 to 0.7 of gestation. Endocrinology. 139:2564-2570.

Riquelme R.A., Sanchez G., Liberona L., Sanhueza E.M., Giussani D.A., Blanco C.E., Hanson M.A., and Llanos A.J. (2002). Nitric oxide plays a role in the regulation of adrenal blood flow and adrenocorticomedullary functions in the llama fetus. J. Physiol. 544:267-276.

Ruijtenbeek K., le Noble F.A.C., Janssen G.M.J., Kessels C.G.A., Fazzi G.E., Blanco C.E., and De Mey J.G.R. (2000). Chronic hypoxia stimulates periarterial sympathetic nerve development in the chick embryo. Circulation. 102(23):2892-2897. 
Salinas C.E., Blanco C.E., Villena M., Camm E.J., Tuckett J.D., Weerakkody R.A., Kane A.D., Shelley A.M., Wooding F.B.P., Quy M., and Giussani D.A. (2010). Cardiac and vascular disease prior to hatching in chick embryos incubated at high altitude. J. Developmental Origins Health Dis. 1:60-66.

Scott T.R., Johnson W.A, Satterlee D.G., and Gildersleeve R.P. (1981). Circulating levels of corticosterone in the serum of developing chick embryos and newly hatched chicks. Poult. Sci. 6:1314-1320.

Seckl J.R., Nyirenda M.J., Walker B.R., and Chapman K.E. (1999). Glucocorticoids and fetal programming. Biochem. Soc. Trans. 27:74-78

Thakor A.S., and Giussani D.A. (2009). Effects of acute acidemia on the fetal cardiovascular defense to acute hypoxemia. Am. J. Physiol. Regul. Integr. Comp. Physiol. 296:R90-R99.

Tintu A., Rouwet E., Verlohren S., Brinkmann J., Ahmad S., Crispi F., van Bilsen M., Carmeliet P., Staff A.C., Tjwa M., Cetin I., Gratacos E., Hernandez-Andrade E., Hofstra L., Jacobs M., Lamers W.H., Morano I., Safak E., Ahmed A., and le Noble F. (2009). Hypoxia induces dilated cardiomyopathy in the chick embryo: mechanism,intervention, and long-term consequences. PLoS One 24(4):e5155.

Vargas V.E., Myers D.M., Kaushal K.M., and Ducsay C.A. (2010). Effects of long term hypoxia cAMP-dependent protein kinase A (PKA) in the regulation of cortisol synthesis in the near term ovine fetal adrenal. Reprod. Sci. 17:3 suppl: pp. 1A$64 \mathrm{~A}$.
Wassermann G.F., and Bernard E.A. (1970). Adrenaline content of the chick embryo adrenal gland during development. Acta Physiol. Latin Am. 20:171-173.

Watterberg K.L., Gerdes J.S., Cole C.H., Aucott S.W., Thilo E.H., Mammel M.C., Couser R.J., Garland J.S., Rozycki H.J., Leach C.L., Backstrom C., and Shaffer M.L. (2004). Prophylaxis of early adrenal insufficiency to prevent bronchopulmonary dysplasia: a multicenter trial. Pediatrics. 114:1649-1657.

West J.B. (1999). Recent advances in human physiology at extreme altitude. Adv.Exp. Med. Biol. 474:287-296.

Wise P.M., and Frye B.E. (1973). Functional development of the hypothalamo-hypophyseal-adrenal cortex axis in the chick embryo, Gallus domesticus. J. Exp. Zool. 185:277-292.

Woods J.E., De Vries G.W, and Thommes R.C. (1971). Ontogenesis of the pituitary-adrenal axis in the chick embryo. Gen. Comp. Endocrinol. 17:407-415.

Address correspondence to: Dino A Giussani, Ph.D. Department of Physiology, Development $\mathcal{E}$ Neuroscience University of Cambridge Downing Street, Cambridge, CB2 3EG, UK

E-mail: dag26@cam.ac.uk

Received June 28, 2010; accepted in final form October 4, 2010. 


\section{Chapter V}

High altitude hypoxia and blood pressure dysregulation in adult chickens.

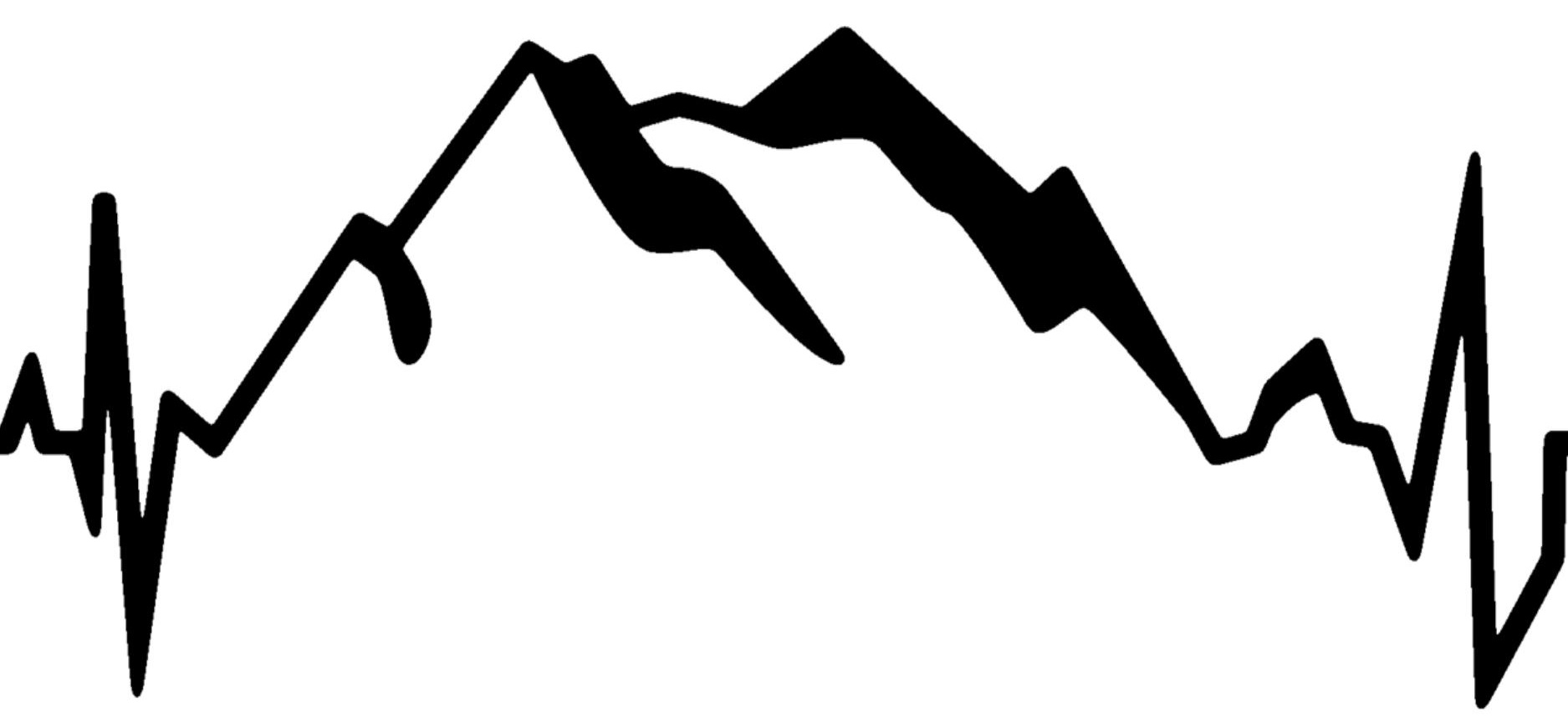

J Dev Orig Health Dis. 2013; 4:69-76 



\title{
High altitude hypoxia and blood pressure dysregulation in adult chickens
}

\author{
E. A. Herrera ${ }^{1}$, C. E. Salinas ${ }^{2}$, C. E. Blanco ${ }^{3}$, M. Villena ${ }^{2}$ and D. A. Giussani ${ }^{4 *}$ \\ ${ }^{1}$ Laboratorio de Función y Reactividad Vascular, Programa de Fisiopatologiá, Instituto de Ciencias Biomédicas, Facultad de Medicina, \\ Universidad de Chile, Santiago, Chile \\ ${ }^{2}$ Instituto Boliviano de Biologia de Altura, Facultad de Medicina, Universidad Mayor de San Andrés, La Paz, Bolivia \\ ${ }^{3}$ National Children's Research Centre, Our Lady's Children's Hospital, Crumlin, Dublin, Ireland \\ ${ }^{4}$ Department of Physiology, Development and Neuroscience, University of Cambridge, Cambridge, UK
}

\begin{abstract}
Although it is accepted that impaired placental perfusion in complicated pregnancy can slow fetal growth and programme an increased risk of cardiovascular dysfunction at adulthood, the relative contribution of reductions in fetal nutrition and in fetal oxygenation as the triggering stimulus remains unclear. By combining high altitude (HA) with the chick embryo model, we have previously isolated the direct effects of HA hypoxia on embryonic growth and cardiovascular development before hatching. This study isolated the effects of developmental hypoxia on cardiovascular function measured in vivo in conscious adult male and female chickens. Chick embryos were incubated, hatched and raised at sea level (SL, nine males and nine females) or incubated, hatched and raised at HA (seven males and seven females). At 6 months of age, vascular catheters were inserted under general anaesthesia. Five days later, basal blood gas status, basal cardiovascular function and cardiac baroreflex responses were investigated. HA chickens had significantly lower basal arterial $\mathrm{PO}_{2}$ and haemoglobin saturation, and significantly higher haematocrit than SL chickens, independent of the sex of the animal. HA chickens had significantly lower arterial blood pressure than SL chickens, independent of the sex of the animal. Although the gain of the arterial baroreflex was decreased in HA relative to SL male chickens, it was increased in HA relative to SL female chickens. We show that development at HA lowers basal arterial blood pressure and alters baroreflex sensitivity in a sex-dependent manner at adulthood.
\end{abstract}

Received 16 August 2012; Revised 18 August 2012; Accepted 20 August 2012; First published online 19 September 2012

Key words: baroreflex, cardiovascular disease, chronic hypoxia, fetal programming

\section{Introduction}

Overwhelming evidence obtained from studies in human links development under sub-optimal intrauterine conditions with increased risk of cardiovascular disease. ${ }^{1}$ Common sub-optimal intrauterine conditions include impaired fetal nutrition and oxygenation. Consequently, many studies have now accumulated to report that nutrient ${ }^{2-4}$ and oxygen ${ }^{2,5-9}$ restriction during development promotes intrauterine growth restriction (IUGR) and programmes cardiovascular dysfunction in the adult offspring.

Several studies, including our own, have used exposure of the pregnant rodent to a prolonged period of hypoxia during gestation and have studied adverse effects on fetal growth and on the cardiovascular system of the offspring at adulthood. These studies have reported that prenatal chronic hypoxia with or without IUGR programmes in the adult offspring impaired cardiac performance with increased susceptibility to cardiac ischaemia/reperfusion injury ${ }^{6-10}$ and pronounced endothelial dysfunction in peripheral resistance

*Address for correspondence: Prof. D. A. Giussani, Department of Physiology, Development and Neuroscience, University of Cambridge, Cambridge CB2 3EG, UK.

(Emaildag26@cam.ac.uk) circulations. ${ }^{8,9,11}$ However, because maternal exposure to hypoxia can lead to a significant decrease in maternal food intake, ${ }^{12,13}$ the extent to which any adverse effects on the offspring are due to fetal undernutrition and/or underoxygenation remain unclear.

More than 140 million people live at altitudes higher than $3000 \mathrm{~m}$, providing the largest single human group at risk for fetal exposure to hypoxia. ${ }^{14}$ Studies of highland populations have reported fetal growth restriction and adverse cardiovascular alterations in offspring of high altitude (HA) relative to those of sea level (SL) pregnancies. ${ }^{15-23}$ However, because most of HA populations are also impoverished, the relative contributions of fetal hypoxia and of malnutrition in slowing fetal growth and programming cardiovascular dysfunction, again, remain uncertain.

The chick embryo provides a useful model to investigate the direct effects on fetal growth and on programming of cardiovascular dysfunction of developmental hypoxia independent of additional effects on the nutrition of the mother and/or on the maternal or placental physiology and/or on lactation. ${ }^{24-26}$ By combining HA exposure with the chick embryo model, we have previously been able to isolate the direct effects of HA hypoxia on fetal growth ${ }^{27}$ and on fetal cardiovascular development. ${ }^{28}$ Incubation of fertilized eggs 
from SL hens at HA promoted growth restriction, cardiomegaly and cardiac and aortic wall thickening in the chick embryo by the end of the incubation period. ${ }^{27,28}$ These effects of HA incubation could be prevented by incubating eggs from HA hens at SL or by incubating eggs from SL hens at HA with oxygen supplementation. ${ }^{27,28}$ In the present study, we have combined HA exposure with the chick embryo model, to isolate the effects of HA hypoxia during embryonic development and postnatal life on cardiovascular function measured in vivo at adulthood. Because in this model the hypoxic environmental condition persists before and after hatching, the study also permits the investigation of the effects of a 'double insult' in triggering cardiovascular dysfunction. As it is established that differences in sex affect developmental origins of cardiovascular disease, ${ }^{29}$ this study investigated both male and female adult chickens.

\section{Methods}

All procedures were approved by the local ethics committee of the Bolivian Institute for High Altitude Biology (Consejo Técnico, IBBA, Universidad Mayor de San Andrés, La Paz, Bolivia) and were performed under the UK Animals (Scientific Procedures) Act 1986.

\section{Animals}

The study was conducted in Bolivia, in the HA city of La Paz (HA, $3600 \mathrm{~m}, 494 \mathrm{mmHg}, \mathrm{PO}_{2} 100 \mathrm{mmHg}$ ) and the SL city of Santa Cruz (SL, $420 \mathrm{~m}, 760 \mathrm{mmHg}, \mathrm{PO}_{2} 160 \mathrm{mmHg}$ ). Eighteen (nine males and nine females) Black Leghorn chicken embryos were incubated, hatched and raised at SL and 14 (seven males and seven females) Black Leghorn chicken embryos were incubated, hatched and raised at HA. At 6 months, which in this species represents early adulthood, the animals were subjected to general anaesthesia $(10 \mathrm{mg} / \mathrm{kg}$ Xylazine 2\%, Millpledge Pharmaceuticals, United Kingdom and $15 \mathrm{mg} / \mathrm{kg}$ Ketamine, Ketaset, Fort Dodge Animal Health, Iowa, USA, i.m.) and the femoral artery and vein were isolated via a hind limb medial incision. Polyvinyl catheters (i.d. $0.58 \mathrm{~mm}$; o.d. $0.96 \mathrm{~mm}$; Critchly Electrical Products, NSW, Australia) were placed in the descending aorta and inferior vena cava. The catheters were filled with heparinized saline (100 i.u. heparin in $0.9 \% \mathrm{NaCl}$ ), plugged with a brass pin and tunnelled subcutaneously to exit between the origin of the wings at the back of the chicken. After surgery, the animals were returned to a recovery room and then to floor pens. At least 5 days of post-operative recovery were allowed before the beginning of any experiment. Catheters were maintained patent by daily flushing with heparinized saline. At this time, an arterial blood sample was taken to monitor well-being.

\section{Experimental protocol}

On the day of experiments, the chicken was placed in a sling inside a dark wooden box with appropriate ventilation.
The catheters were extended. The arterial catheter was connected to a pressure transducer (COBE; Argon Division, Maxxim Medical, Athens, Texas, USA) at the level of the base of the heart. Mean (MAP), systolic (SAP) and diastolic arterial pressure (DAP) and heart rate (HR) were recorded continuously via a data acquisition system (MPAQ - Maastricht Programmable AcQuisition system, Maastricht Instruments, The Netherlands, $500 \mathrm{~Hz}$ sample rate).

During basal recording, before the start of any experiment, $0.5 \mathrm{ml}$ of arterial blood was taken to determine $\mathrm{pHa}, \mathrm{PaO}_{2}$ and $\mathrm{PaCO}_{2}$ (ABL 500, Radiometer, Copenhagen, Denmark, measurements corrected to $37^{\circ} \mathrm{C}$ ), percentage saturation of haemoglobin $\left(\mathrm{SaO}_{2}\right)$ and haematocrit (Ht; OSM3, Radiometer, Copenhagen, Denmark).

The chicken was then subjected to a protocol consisting of a 10-min period of baseline (B), a 10-min intravenous infusion (I) and 10-min period of recovery (R). The infusion consisted of either phenylephrine $(50 \mu \mathrm{g} / \mathrm{kg}$ per min) or sodium nitroprusside $(20 \mu \mathrm{g} / \mathrm{kg}$ per min) in separate experiments. The experiments were performed on the same day with at least $2 \mathrm{~h}$ between them to allow the cardiovascular variables to return to basal values. The phenylephrine experiment always preceded the infusion of nitroprusside.

At the end of the experiments, the chicken was humanely killed with an overdose of anaesthetic $(100 \mathrm{mg} / \mathrm{kg}$, Thiopental injection BP, Link Pharmaceuticals Ltd, UK, i.v.). Post mortem, the chicken was weighed and several body measurements were taken.

\section{Data and statistical analyses}

Baseline values for MAP, HR, pulse interval (PI; HR/60,000) and the rate pressure product [RPP; $(\mathrm{HR} \times \mathrm{SAP}) / 1000]$ were calculated. In brief, the calculation of the baroreflex gain required two steps. The first step was the calculation of the slope of the HR-blood pressure relationship and the maximum and minimum values for $\mathrm{HR}$ for each animal. This was achieved by plotting the minute-by-minute HR and blood pressure responses during the beginning of the infusion period from baseline to plateau. Only the period from baseline to plateau is used to avoid confounding by resetting of the baroreflex. Arterial blood pressure and HR responses to the drugs were fitted to sigmoidal curves as follows: $\mathrm{HR}=\mathrm{HR}_{\min }+$ $\left(\left(\mathrm{HR}_{\max }-\mathrm{HR}_{\min }\right) /\left(1+10^{\text {(Mid-point-MAP) }} \times\right.\right.$ Gain coefficient $)$. The value for gain represents the gain coefficient of the Hill slope that describes the steepness of the curve. The second step was to calculate the baroreflex sensitivity according to McDowall and Dampney ${ }^{30}$ applying the values for gain, maximum and minimum $\mathrm{HR}$ to the following equation: Baroreflex Gain = $\left(\left(\mathrm{HR}_{\max }-\mathrm{HR}_{\min }\right) \times \mathrm{Gain}\right.$ coefficient $) / 4$. This was done for each individual animal and thereafter the mean \pm S.E.M. of the baroreflex gain for each group was calculated.

All data are expressed as mean \pm S.E.M. Comparisons between groups were assessed statistically using two-way ANOVA with the Student-Newman-Keuls post-hoc test, with altitude and sex 
as factors (Prism 5, GraphPad Software, Inc.). For all comparisons, statistical significance was accepted when $P<0.05$.

\section{Results}

\section{Arterial blood gas status}

There were no differences in basal arterial $\mathrm{pH}$ and $\mathrm{pCO}_{2}$ between groups. However, HA chickens had lower arterial $\mathrm{pO}_{2}, \mathrm{SaO}_{2}$ and increased Ht compared with SL chickens (Fig. 1). There was no effect of sex on arterial blood gas status either at SL or at HA. Therefore, these data were grouped at each altitude (Fig. 1).

\section{In vivo basal cardiovascular function}

Values for SAP, DAP and MAP during basal conditions were significantly lower in HA compared with SL chickens, and these differences were independent of the sex of the animal
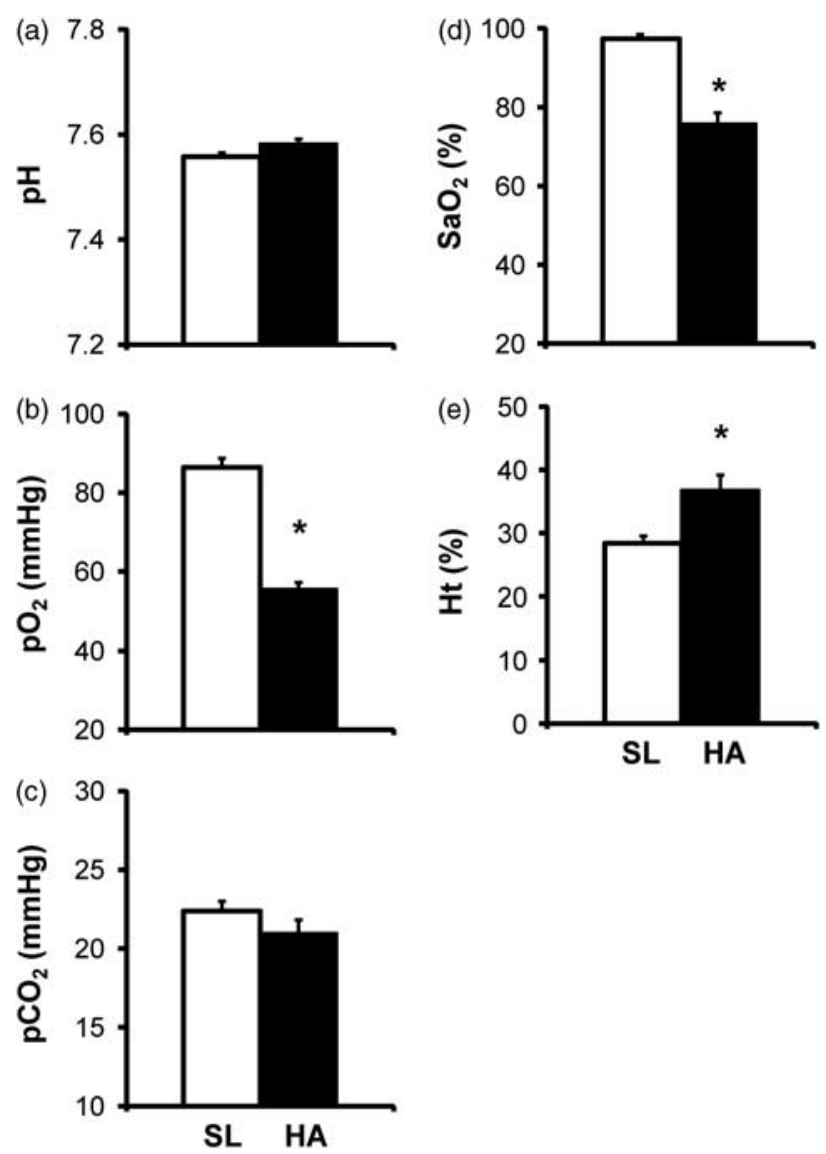

Fig. 1. Arterial blood gas status in sea level (SL) and high altitude (HA) adult chickens. Values are the mean \pm S.E.M. for $\mathrm{pH}(a), \mathrm{pO}_{2}(b)$, $\mathrm{pCO}_{2}(c), \mathrm{O}_{2}$ haemoglobin saturation $\left(d, \mathrm{SaO}_{2}\right)$ and haematocrit $(e, \mathrm{Ht})$ in nine males and nine female chickens incubated, hatched and raised at SL $(\square)$ and in seven male and seven female chickens incubated, hatched and raised at HA ( $\mathbf{\square})$. There was no effect of sex on arterial blood gas status either at SL or at HA. Therefore, these data were grouped at each altitude. Significant differences $(P<0.05)$ are: ${ }^{*} \mathrm{SL}$ $v$. HA (Two-way ANOVA + Student-Newman-Keuls post-hoc test).
(Fig. 2a-2c). In contrast, basal HR was significantly higher and basal PI significantly lower in female than in male chickens at SL. Although basal HR and PI were significantly altered in HA males compared with SL males, there was no effect of altitude on basal HR or PI in female chickens (Fig. 2d and 2e). The RPP, an index of myocardial workload and oxygen consumption, ${ }^{31}$ was significantly elevated during basal conditions in female chickens compared with male chickens at SL. Although HA had no effect of RPP in males, values for RPP in HA females were significantly lower compared with SL females (Fig. 2f).

\section{In vivo baroreflex function}

Independent of sex and altitude, all animals responded with reciprocal changes in HR to increases and decreases in arterial
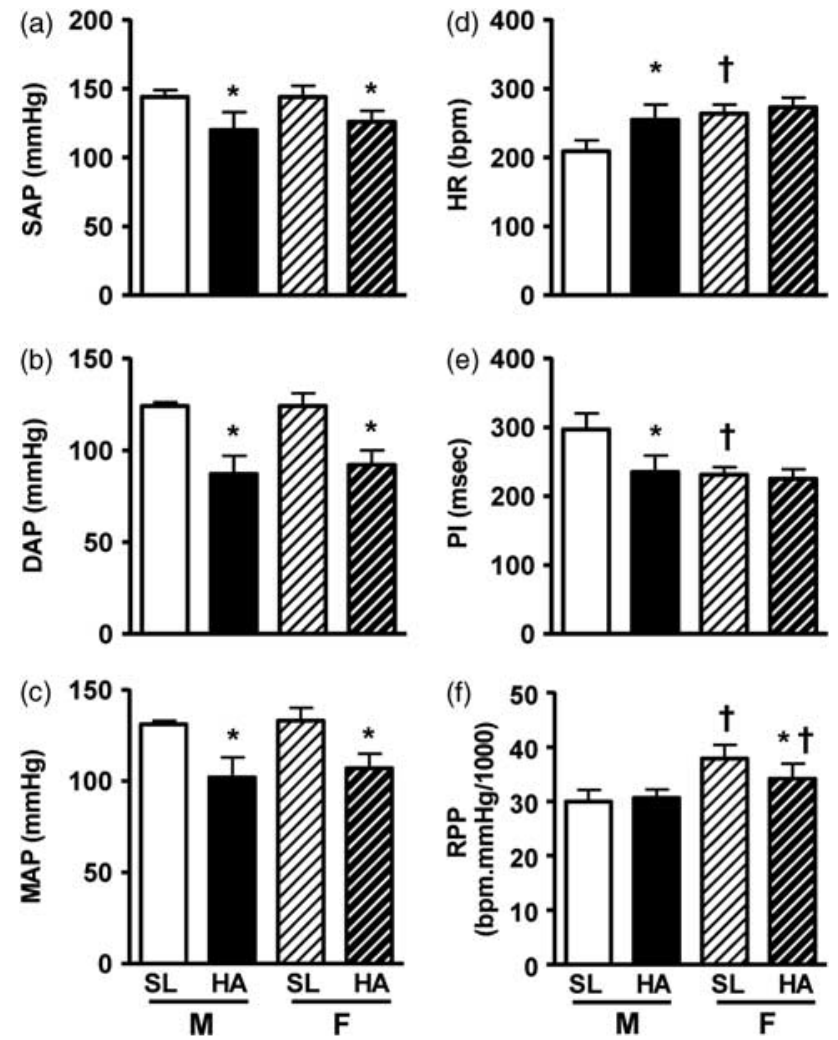

Fig. 2. Basal cardiovascular function in sea level (SL) and high altitude (HA) adult chickens. Values are the mean \pm s.E.M. for systolic arterial pressure ( $a, \mathrm{SAP})$, diastolic arterial pressure $(b, \mathrm{DAP})$, mean arterial pressure $(c, \mathrm{MAP})$, heart rate $(d, \mathrm{HR})$, pulse interval $(e, \mathrm{PI})$ and the rate-pressure product $(f, \mathrm{RPP})$ in nine males and nine female chickens incubated, hatched and raised at SL (white solid and white hatched bars) and in seven male and seven female chickens incubated, hatched and raised at HA (black solid and black hatched bars). Significant differences $(P<0.05)$ are: ${ }^{\dagger}$ male $v$. female, same altitude (hypoxia independent of sex) or ${ }^{*} \mathrm{SL} v$. HA, for same sex (sex independent of hypoxia). There was no interaction between hypoxia and sex (Two-way ANOVA + Student-Newman-Keuls post-hoc test). 

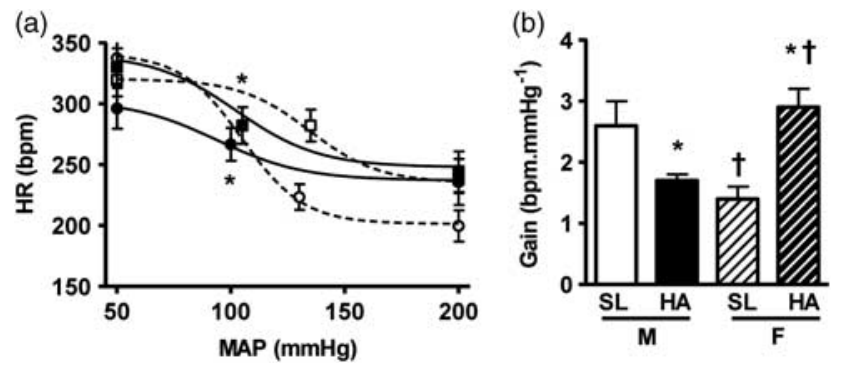

Fig. 3. Baroreflex function in sea level (SL) and high altitude (HA) adult chickens. Values are the mean \pm S.E.M. for cardiac baroreflex function curves $(a)$ and the gain of the cardiac baroreflex $(b)$ in nine males and nine female chickens incubated, hatched and raised at SL and in seven male and seven female chickens incubated, hatched and raised at HA. Groups are sea level males (white circles, white bars), HA males (black circles, black bars), SL females (white squares, white hatched bars) and HA females (black squares, black hatched bars) chickens. Significant differences $(P<0.05)$ are: ${ }^{\dagger}$ male $v$. female, same altitude (hypoxia independent of sex) or *SL $v$. HA, for same sex (sex independent of hypoxia). There was no interaction between hypoxia and sex (Two-way ANOVA + Student-Newman-Keuls post-hoc test). The calculation of the baroreflex gain required two steps. The first step was the calculation of the slope of the heart rate (HR)-blood pressure relationship and the maximum and minimum values for HR for each animal. This was achieved by plotting the minute-by-minute HR and blood pressure responses during the beginning of the infusion period from baseline to plateau. Only the period from baseline to plateau is used to avoid confounding by resetting of the baroreflex. Arterial blood pressure and $\mathrm{HR}$ responses to the drugs were fitted to sigmoidal curves as follows: $\mathrm{HR}=\mathrm{HR}_{\min }+\left(\left(\mathrm{HR}_{\max }-\mathrm{HR}_{\min }\right) /\left(1+10^{\text {(Mid-point-MAP })}\right.\right.$ $\times$ Gain coefficient). The value for gain represents the gain coefficient of the Hill slope that describes the steepness of the curve. The second step was to calculate the baroreflex sensitivity according to McDowall and Dampney ${ }^{30}$ applying the values for gain, maximum and minimum HR to the following equation: Baroreflex Gain $=\left(\left(\mathrm{HR}_{\max }-\mathrm{HR}_{\min }\right) \times\right.$ Gain coefficient $) / 4$,

The three points with S.E.M. on the curve represent the summary measures of the analysis: the gain and maximum and minimum $\mathrm{HR}$ values.

blood pressure induced by the administration of phenylephrine and sodium nitroprusside, respectively. Although values representing the cardiac baroreflex gain were significantly depressed in HA males compared with SL males, baroreflex gain was markedly increased in HA females compared with SL females (Fig. 3).

\section{Biometry}

Body weight was significantly lower in females than in males either at SL or HA. Body length [crown-rump length (CRL)] and other longitudinal measurements also tended to be shorter in females than in males at either SL or HA; however, only tibial and meta-tarsal length reached significant differences in SL animals (Table 1). Values for ponderal index were higher in male and female chickens at HA; however, the mean calculation was not significantly different from SL. The head length:CRL ratio was significantly elevated in HA compared with SL male but not female chickens (Table 1). The same trend was observed for the head diameter:body weight ratio, but the difference in males did not reach significance.

\section{Discussion}

By combining the chick embryo model with incubation at HA, we have previously isolated the effects of developmental hypoxia on fetal growth and on fetal cardiac and aortic wall remodelling. ${ }^{27,28}$ Here, we isolate the effects of developmental hypoxia on cardiovascular function at adulthood measured in conscious chickens in vivo, and show that development at HA lowers basal arterial blood pressure and alters baroreflex sensitivity in a sex-dependent manner at adulthood.

Several experimental models of adverse intrauterine conditions, including maternal protein deprivation, ${ }^{32-38}$ maternal overnutrition ${ }^{39-43}$ and excess glucocorticoid exposure ${ }^{44-47}$ have been reported to programme an increase in basal arterial blood pressure at adulthood. By contrast, there is little information on the effects of developmental hypoxia on the regulation of arterial blood pressure at adulthood, measured in vivo, particularly in the absence of anaesthesia. This is surprising as fetal hypoxia is the most common challenge in complicated pregnancy, such as during preeclampsia ${ }^{48}$ and placental insufficiency. ${ }^{49}$ The present study shows that developmental hypoxia is associated with a fall, rather than an increase, in mean, systolic and diastolic basal arterial blood pressure in adult conscious chickens and that this effect is independent of the sex of the animal. Given that hypotension occurred in the absence of bradycardia during basal conditions, an effect on lowering peripheral vascular resistance of development at HA is favoured. A lack of a programmed hypertensive effect in adult chickens of isobaric hypoxia in ovo has also been reported by Ruijtenbeek et al. ${ }^{50}$ Similar findings have been reported in chick ${ }^{51}$ and alligator ${ }^{52}$ embryos incubated under chronic isobaric hypoxia, yielding basal hypotension without basal bradycardia, even before hatching. Coney and Marshall $^{53}$ reported that adult rats of chronically hypoxic pregnancies had significantly elevated resting femoral blood flow compared with adult rats of normoxic pregnancies, supporting a programmed fall in peripheral vascular resistance contributing to the programmed hypotensive effects of developmental hypoxia. Further, Herrera et $a l^{54}$ reported that HA chronic hypoxia during gestation yielded newborn lambs with significantly lower systemic vascular resistance than lowland newborn lambs, although basal systemic arterial blood pressure was not different. The mechanism via which developmental hypoxia may promote a fall in peripheral vascular resistance and a fall in resting blood pressure at adulthood is unclear. However, there is an established relationship between increased plasma $\mathrm{Ht}$, blood viscosity and shear stress-induced increases in nitric oxide (NO) bioavailability. ${ }^{55}$ Interestingly, moderate elevations in blood viscosity by increasing $\mathrm{Ht}$ by $10 \%$ from baseline could produce reductions 
Table 1. Body weight and dimensions in SL and HA adult chickens

\begin{tabular}{|c|c|c|c|c|}
\hline & \multicolumn{2}{|c|}{ Males } & \multicolumn{2}{|c|}{ Females } \\
\hline & SL & HA & SL & $\mathrm{HA}$ \\
\hline Body (g) & $2012 \pm 159$ & $1945 \pm 341$ & $1521 \pm 76^{\dagger}$ & $1614 \pm 99$ \\
\hline CRL (mm) & $485 \pm 15$ & $436 \pm 30$ & $424 \pm 14$ & $393 \pm 13$ \\
\hline Femur $(\mathrm{mm})$ & $135 \pm 4$ & $138 \pm 11$ & $129 \pm 5$ & $123 \pm 4$ \\
\hline Tibia (mm) & $166 \pm 5$ & $152 \pm 6$ & $141 \pm 5^{\dagger}$ & $131 \pm 8$ \\
\hline Meta-tarsal (mm) & $124 \pm 4$ & $113 \pm 7$ & $98 \pm 4^{\dagger}$ & $100 \pm 5$ \\
\hline $\mathrm{HD}(\mathrm{mm})$ & $33.1 \pm 1.2$ & $31.1 \pm 0.9$ & $30.1 \pm 0.7$ & $28.8 \pm 1.1$ \\
\hline $\mathrm{HL}(\mathrm{mm})$ & $75 \pm 1$ & $78 \pm 2$ & $74 \pm 1$ & $75 \pm 2$ \\
\hline Beak (mm) & $34.0 \pm 2.8$ & $37.5 \pm 1.0$ & $35.7 \pm 1.1$ & $35.4 \pm 0.7$ \\
\hline Ponderal index $\left(\mathrm{kg} / \mathrm{m}^{3}\right)$ & $19.01 \pm 1.30$ & $24.47 \pm 4.76$ & $22.30 \pm 1.85$ & $27.27 \pm 2.43$ \\
\hline HL:CRL & $0.155 \pm 0.005$ & $0.183 \pm 0.010^{*}$ & $0.176 \pm 0.006$ & $0.192 \pm 0.009$ \\
\hline HD:BW & $0.016 \pm 0.001$ & $0.018 \pm 0.002$ & $0.018 \pm 0.001$ & $0.018 \pm 0.001$ \\
\hline
\end{tabular}

SL, sea level; HA, high altitude; CRL, crown-rump length; HD, head diameter; HL, head length; BW, body weight. Values are mean \pm s.E.M. for BW, CRL, femur, tibia and meta-tarsal lengths, HD, HL, beak length, ponderal index, the ratio of HL:CRL and of HD:BW in nine males and nine female chickens incubated, hatched and raised at SL and in seven male and seven female chickens incubated, hatched and raised at HA. Significant differences $(P<0.05)$ are: $†$ male $v$. female, same altitude or *SL $v$. HA (Two-way ANOVA + Student-Newman-Keuls post-hoc test).

in basal blood pressure by $10 \mathrm{mmHg}$, an effect that could be abolished by treatment with the NO synthase blocker L-N omega-nitro-L-arginine methyl ester (L-NAME) or in NO synthase-deficient mice. ${ }^{56}$ In the present study, chickens at $\mathrm{HA}$ had significantly lower arterial $\mathrm{PO}_{2}$ and haemoglobin oxygen saturation and a persistent increase in $\mathrm{Ht}$ of ca. $8 \%$.

Investigation of the effects of adverse intrauterine conditions on baroreflex function in the offspring is scarce relative to reported programmed changes in basal arterial blood pressure. Within the few studies available, it is known that maternal undernutrition can increase the set point and blunt baroreflex responses in the adult offspring ${ }^{57-59}$ and that embryonic and fetal exposure to excess glucocorticoids can increase the set point and decrease the sensitivity of the baroreflex in fetal, newborn and adult life. ${ }^{44,46,60-62}$ By contrast, as with effects on basal arterial blood pressure, there is less information on the effects of developmental hypoxia on baroreflex function. One study has reported that chronic hypoxic pregnancy decreases baroreflex sensitivity in the lategestation ovine fetus ${ }^{63}$ and Peyronnet et al. ${ }^{64}$ predicted but did not measure alterations in baroreflex function in rat adult offspring of chronically hypoxic pregnancies.

Gilbert and Nijland, ${ }^{65}$ in an elegant and useful review, brought to attention the growing body of evidence reporting sex differences in the developmental programming of alterations in arterial blood pressure and its regulation. Again, most of this evidence comes from studies involving alterations in maternal nutrition ${ }^{2,62,66}$ or from models of maternal stress and glucocorticoid excess ${ }^{62,67}$ than from models of prenatal hypoxia. The weight of the evidence has generally, but not exclusively, suggested that female offspring are less sensitive in manifestation of cardiovascular disease caused by prenatal stimuli. ${ }^{65}$ Accordingly, Davidge and colleagues have reported that peripheral vascular function and cardiac hypertrophy is more profoundly affected in male than in female rat offspring of hypoxic pregnancies, particularly as they age. ${ }^{68-70}$ Similarly, prenatal hypoxia caused an increase in heart susceptibility to ischaemia reperfusion injury in male but not in female adult rat offspring. ${ }^{71}$ To our knowledge, only one study has reported sexually dimorphic effects in programming changes in baroreflex function induced by maternal malnutrition or fetal glucocorticoid exposure. ${ }^{62}$ Certainly, there have been no reports on sex-dependent programming of baroreflex dysfunction by developmental hypoxia. In the present study, we show that while the effects of developmental hypoxia on resting arterial blood pressure at adulthood were sex independent, development at HA had reciprocal effects on the gain of the baroreflex in adult male and female chickens. Baroreflex gain was decreased in adult males, but it was increased in adult females at HA relative to SL controls. A decrease in baroreflex gain may render the male less able to respond to alterations in blood pressure homoeostasis. Further, the rate-pressure product, an index of myocardial workload and oxygen consumption, ${ }^{31}$ although unchanged in males by altitude, was decreased in highland relative to lowland females. Finally, the head:CRL ratio, an index of blood flow redistribution, although unchanged in females by altitude, was increased in highland relative to lowland males. Collectively, therefore, the present data also suggest a greater resilience of the female offspring to development under conditions of HA hypoxia, again adding to the increasing body of evidence showing greater susceptibility to programmed cardiovascular dysfunction in male than female offspring. It is important to acknowledge that there are 
important differences in sexual differentiation in avian and mammalian species. For instance, in the chicken, males rather than females are homogametic and sex determination is cell autonomous. Therefore, translation of the sexually dimorphic results between species should be considered cautiously.

It has been suggested that it is the mismatch between the pre- and postnatal environment that renders the offspring susceptible to developing disease at adulthood. ${ }^{72}$ In the present study, the environmental condition of hypoxia occurs in ovo and persists after hatching. In this context, it is of interest that significant alterations in blood pressure homoeostasis were evident in male and female adult chickens despite matching of the incubation and post-hatching environments. Cardiovascular dysfunction may therefore have developed in response to a double insult; one occurring before and one after hatching. The partial contributions of incubation $v$. posthatching HA hypoxia in triggering cardiovascular dysfunction at adulthood await investigation by study of adult offspring raised at SL following HA incubation, and by study of adult offspring raised at HA following SL incubation. However, evidence of similar alterations in blood pressure homoeostasis than those reported here at adulthood in the chronically hypoxic chick embryo even before hatching ${ }^{51}$ supports a primary effect triggered during the incubation period rather than after hatching.

In conclusion, by combining the chick embryo model with incubation at HA, we have isolated the effects of developmental hypoxia on cardiovascular function at adulthood measured in conscious chickens in vivo, and show that development at HA promotes blood pressure dysregulation, altering baroreflex sensitivity in a sex-dependent manner at adulthood.

\section{Acknowledgements}

This work was supported by The British Heart Foundation, The Royal Society and the U-Apoya Program, Universidad de Chile. Emilio A. Herrera is a fellow of Beca Presidente de la Republica (CONICYT) from the Chilean Government. Dino A. Giussani is a Royal Society Wolfson Research Merit Award holder.

\section{References}

1. Barker DJ. In utero programming of chronic disease. Clin Sci (Lond). 1998; 95, 115-128.

2. Fowden AL, Giussani DA, Forhead AJ. Intrauterine programming of physiological systems: causes and consequences. Physiology (Bethesda). 2006; 21, 29-37.

3. Gluckman PD, Hanson MA, Cooper C, Thornburg KL. Effect of in utero and early-life conditions on adult health and disease. $N$ Engl J Med. 2008; 359, 61-73.

4. Martin-Gronert MS, Ozanne SE. Mechanisms underlying the developmental origins of disease. Rev Endocr Metab Disord. 2012; 13, 85-92.

5. Thompson LP. Effects of chronic hypoxia on fetal coronary responses. High Alt Med Biol. 2003; 4, 215-224.
6. Hauton D, Ousley V. Prenatal hypoxia induces increased cardiac contractility on a background of decreased capillary density. BMC Cardiovasc Disord. 2009; 9, 1.

7. Patterson AJ, Zhang L. Hypoxia and fetal heart development. Curr Mol Med. 2010; 10, 653-666.

8. Rueda-Clausen CF, Morton JS, Davidge ST. The early origins of cardiovascular health and disease: who, when, and how. Semin Reprod Med. 2011; 29, 197-210.

9. Giussani DA, Camm EJ, Niu Y, et al. Developmental programming of cardiovascular dysfunction by prenatal hypoxia and oxidative stress. PLoS One. 2012; 7, e31017.

10. Li G, Xiao Y, Estrella JL, et al. Effect of fetal hypoxia on heart susceptibility to ischemia and reperfusion injury in the adult rat. J Soc Gynecol Investig. 2003; 10, 265-274.

11. Morton JS, Rueda-Clausen CF, Davidge ST. Mechanisms of endothelium-dependent vasodilation in male and female, young and aged offspring born growth restricted. Am J Physiol Regul Integr Comp Physiol. 2010; 298, R930-R938.

12. de Grauw TJ, Myers RE, Scott WJ. Fetal growth retardation in rats from different levels of hypoxia. Biol Neonate. 1986; 49, 85-89.

13. Camm EJ, Hansell JA, Kane AD, et al. Partial contributions of developmental hypoxia and undernutrition to prenatal alterations in somatic growth and cardiovascular structure and function. Am J Obstet Gynecol. 2010; 203(5), 495.e24-34.

14. Moore LG, Charles SM, Julian CG. Humans at high altitude: hypoxia and fetal growth. Respir Physiol Neurobiol. 2011; 178, 181-190.

15. Lichty JA, Ting RY, Bruns P, Dyer E. Studies of babies born at high altitude. 1. Relation of altitude to birth weight. Am J Dis Child. 1957; 93, 666-669.

16. McClung J. Effects of High Altitude on Human Birth, 1969. Harvard University Press: Cambridge.

17. Haas JD, Frongillo EF, Stepcik C, Beard J, Hurtado L. Altitude, ethnic and sex differences in birthweight and length in Bolivia. Hum Biol. 1980; 52, 459-477.

18. Giussani DA, Phillips PS, Anstee S, Barker DJ. Effects of altitude vs. economic status on birth weight and body shape at birth. Ped Res. 2001; 49, 490-494.

19. Zamudio S, Postigo L, Illsley NP, et al. Maternal oxygen delivery is not related to altitude- and ancestry-associated differences in human fetal growth. J Physiol. 2007; 582(Pt 2), 883-895.

20. Niermeyer S. Cardiopulmonary transition in the high altitude infant. High Alt Med Biol. 2003; 4, 225-239.

21. Peñaloza D, Arias-Stella J, Sime F, Recavarren S, Marticorena E. The heart and pulmonary circulation in children at high altitudes: physiological, anatomical, and clinical observations. Pediatrics. 1964; 34, 568-582.

22. Peñaloza D, Sime F, Ruiz L. Pulmonary hemodynamics in children living at high altitudes. High Alt Med Biol. 2008; 9, 199-207.

23. Huicho L, Niermeyer S. Cardiopulmonary pathology among children resident at high altitude in Tintaya, Peru: a crosssectional study. High Alt Med Biol. 2006; 7, 168-179.

24. Ruijtenbeek K, De Mey JG, Blanco CE. The chicken embryo in developmental physiology of the cardiovascular system: a traditional model with new possibilities. Am J Physiol Regul Integr Comp Physiol. 2002; 283, R549-R550. 
25. Miller SL, Green LR, Peebles DM, Hanson MA, Blanco CE. Effects of chronic hypoxia and protein malnutrition on growth in the developing chick. Am J Obstet Gynecol. 2002; 186, 261-267.

26. Tintu A, Rouwet E, Verlohren S, et al. Hypoxia induces dilated cardiomyopathy in the chick embryo: mechanism, intervention, and long-term consequences. PLoS One. 2009; 4, e5155.

27. Giussani DA, Salinas CE, Villena M, Blanco CE. The role of oxygen in prenatal growth: studies in the chick embryo. J Physiol. 2007; 585(Pt 3), 911-917.

28. Salinas CE, Blanco CE, Villena M, et al. Developmental origin of cardiac and vascular disease in chick embryos incubated at high attitude. J DOHaD. 2010; 1, 60-66.

29. Gilbert JS, Nijland MJ. Sex differences in the developmental origins of hypertension and cardiorenal disease. Am J Physiol Regul Integr Comp Physiol. 2008; 295, R1941-R1952.

30. McDowall LM, Dampney RA. Calculation of threshold and saturation points of sigmoidal baroreflex function curves. Am J Physiol Heart Circ Physiol. 2006; 291, H2003-H2007.

31. Gobel FL, Norstrom LA, Nelson RR, Jorgensen CR, Wang Y. The rate-pressure product as an index of myocardial oxygen consumption during exercise in patients with angina pectoris. Circulation. 1978; 57, 549-556.

32. Langley SC, Jackson AA. Increased systolic blood pressure in adult rats induced by fetal exposure to maternal low protein diets. Clin Sci (Lond). 1994; 86, 217-222.

33. Woodall SM, Johnston BM, Breier BH, Gluckman PD. Chronic maternal undernutrition in the rat leads to delayed postnatal growth and elevated blood pressure of offspring. Pediatr Res. 1996; 40, 438-443.

34. Gardner DS, Jackson AA, Langley-Evans SC. Maintenance of maternal diet-induced hypertension in the rat is dependent on glucocorticoids. Hypertension. 1997; 30, 1525-1530.

35. Manning J, Vehaskari VM. Low birth weight-associated adult hypertension in the rat. Pediatr Nephrol. 2001; 16, 417-422.

36. Gilbert JS, Lang AL, Grant AR, Nijland MJ. Maternal nutrient restriction in sheep: hypertension and decreased nephron number in offspring at 9 months of age. J Physiol. 2005; 565(Pt 1), 137-147.

37. Cambonie G, Comte B, Yzydorczyk C, et al. Antenatal antioxidant prevents adult hypertension, vascular dysfunction, and microvascular rarefaction associated with in utero exposure to a low-protein diet. Am J Physiol Regul Integr Comp Physiol. 2007; 292, R1236-R1245.

38. Watkins AJ, Wilkins A, Cunningham C, et al. Low protein diet fed exclusively during mouse oocyte maturation leads to behavioural and cardiovascular abnormalities in offspring. J Physiol. 2008; 586, 2231-2244.

39. Langley-Evans SC, Clamp AG, Grimble RF, Jackson AA. Influence of dietary fats upon systolic blood pressure in the rat. Int J Food Sci Nutr. 1996; 47, 417-425.

40. Langley-Evans SC. Intrauterine programming of hypertension in the rat: nutrient interactions. Comp Biochem Physiol A Physiol. 1996; 114, 327-333.

41. Khan IY, Taylor PD, Dekou V, et al. Gender-linked hypertension in offspring of lard-fed pregnant rats. Hypertension. 2003; 41, 168-175.

42. Armitage JA, Lakasing L, Taylor PD, et al. Developmental programming of aortic and renal structure in offspring of rats fed fat-rich diets in pregnancy. J Physiol. 2005; 565(Pt 1), 171-184.
43. Elahi MM, Cagampang FR, Anthony FW, et al. Statin treatment in hypercholesterolemic pregnant mice reduces cardiovascular risk factors in their offspring. Hypertension. 2008; 51, 939-944.

44. Dodic M, Abouantoun T, O'Connor A, Wintour EM, Moritz KM. Programming effects of short prenatal exposure to dexamethasone in sheep. Hypertension. 2002; 40, 729-734.

45. Ortiz LA, Quan A, Zarzar F, Weinberg A, Baum M. Prenatal dexamethasone programs hypertension and renal injury in the rat. Hypertension. 2003; 41, 328-334.

46. Shaltout HA, Rose JC, Figueroa JP, et al. Acute AT(1)-receptor blockade reverses the hemodynamic and baroreflex impairment in adult sheep exposed to antenatal betamethasone. Am J Physiol Heart Circ Physiol. 2010; 299, H541-H547.

47. Roghair RD, Wemmie JA, Volk KA, et al. Maternal antioxidant blocks programmed cardiovascular and behavioural stress responses in adult mice. Clin Sci (Lond). 2011; 121, 427-436.

48. Soleymanlou N, Jurisica I, Nevo O, et al. Molecular evidence of placental hypoxia in preeclampsia. J Clin Endocrinol Metab. 2005; 90, 4299-4308.

49. Baschat AA. Fetal responses to placental insufficiency: an update. BJOG. 2004; 111, 1031-1041.

50. Ruijtenbeek K, Kessels CG, Janssen BJ, et al. Chronic moderate hypoxia during in ovo development alters arterial reactivity in chickens. Pflugers Arch. 2003; 447, 158-167.

51. Lindgren I, Crossley D II, Villamor E, Altimiras J. Hypotension in the chronically hypoxic chicken embryo is related to the $\beta$-adrenergic response of chorioallantoic and femoral arteries and not to bradycardia. Am J Physiol Regul Integr Comp Physiol. 2011; 301, R1161-R1168.

52. Crossley DA II, Altimiras J. Cardiovascular development in embryos of the American alligator Alligator mississippiensis: effects of chronic and acute hypoxia. J Exp Biol. 2005; 208(Pt 1), 31-39.

53. Coney AM, Marshall JM. Effects of maternal hypoxia on muscle vasodilatation evoked by acute systemic hypoxia in adult rat offspring: changed roles of adenosine and A1 receptors. J Physiol. 2010; 588(Pt 24), 5115-5125.

54. Herrera EA, Pulgar VM, Riquelme RA, et al. High-altitude chronic hypoxia during gestation and after birth modifies cardiovascular responses in newborn sheep. Am J Physiol Regul Integr Comp Physiol. 2007; 292, R2234-R2240.

55. Martini J, Carpentier B, Negrete AC, Frangos JA, Intaglietta M. Paradoxical hypotension following increased hematocrit and blood viscosity. Am J Physiol Heart Circ Physiol. 2005; 289, H2136-H2143.

56. Martini J, Carpentier B, Chávez Negrete A, et al. Beneficial effects due to increasing blood and plasma viscosity. Clin Hemorheol Microcirc. 2006; 35, 51-57.

57. Pladys P, Lahaie I, Cambonie G, et al. Role of brain and peripheral angiotensin II in hypertension and altered arterial baroreflex programmed during fetal life in rat. Pediatr Res. 2004; 55, 1042-1049.

58. Gardner DS, Pearce S, Dandrea J, et al. Peri-implantation undernutrition programs blunted angiotensin II evoked baroreflex responses in young adult sheep. Hypertension. 2004; 43, 1290-1296.

59. Gopalakrishnan GS, Gardner DS, Rhind SM, et al. Programming of adult cardiovascular function after early maternal undernutrition in sheep. Am J Physiol Regul Integr Comp Physiol. 2004; 287, R12-R20. 
60. Fletcher AJ, McGarrigle HH, Edwards CM, Fowden AL, Giussani DA. Effects of low dose dexamethasone treatment on basal cardiovascular and endocrine function in fetal sheep during late gestation. J Physiol. 2002; 545(Pt 2), 649-660.

61. Segar JL, Roghair RD, Segar EM, et al. Early gestation dexamethasone alters baroreflex and vascular responses in newborn lambs before hypertension. Am J Physiol Regul Integr Comp Physiol. 2006; 291, R481-R488.

62. Roghair RD, Segar JL, Volk KA, et al. Vascular nitric oxide and superoxide anion contribute to sex-specific programmed cardiovascular physiology in mice. Am J Physiol Regul Integr Comp Physiol. 2009; 296, R651-R662.

63. Pulgar VM, Hong JK, Jessup JA, et al. Mild chronic hypoxemia modifies expression of brain stem angiotensin peptide receptors and reflex responses in fetal sheep. Am J Physiol Regul Integr Comp Physiol. 2009; 297, R446-R452.

64. Peyronnet J, Dalmaz Y, Ehrström M, et al. Long-lasting adverse effects of prenatal hypoxia on developing autonomic nervous system and cardiovascular parameters in rats. Pflugers Arch. 2002; 443, 858-865.

65. Gilbert JS, Nijland MJ. Sex differences in the developmental origins of hypertension and cardiorenal disease. Am J Physiol Regul Integr Comp Physiol. 2008; 295, R1941-R1952.
66. do Carmo Pinho Franco M, Nigro D, Fortes ZB, et al. Intrauterine undernutrition - renal and vascular origin of hypertension. Cardiovasc Res. 2003; 60, 228-234.

67. Igosheva N, Taylor PD, Poston L, Glover V. Prenatal stress in the rat results in increased blood pressure responsiveness to stress and enhanced arterial reactivity to neuropeptide $Y$ in adulthood. J Physiol. 2007; 582(Pt 2), 665-674.

68. Hemmings DG, Williams SJ, Davidge ST. Increased myogenic tone in 7-month-old adult male but not female offspring from rat dams exposed to hypoxia during pregnancy. Am J Physiol Heart Circ Physiol. 2005; 289, H674-H682.

69. Rueda-Clausen CF, Morton JS, Davidge ST. Effects of hypoxiainduced intrauterine growth restriction on cardiopulmonary structure and function during adulthood. Cardiovasc Res. 2009; 81, 713-722.

70. Morton JS, Rueda-Clausen CF, Davidge ST. Flow-mediated vasodilation is impaired in adult rat offspring exposed to prenatal hypoxia. J Appl Physiol. 2011; 110, 1073-1082.

71. Xue Q, Zhang L. Prenatal hypoxia causes a sex-dependent increase in heart susceptibility to ischemia and reperfusion injury in adult male offspring: role of protein kinase $\mathrm{C}$ epsilon. J Pharmacol Exp Ther. 2009; 330, 624-632.

72. Gluckman PD, Hanson MA. Mismatch: the lifestyle diseases timebomb. Oxford University Press. 2006; 6, 137-211. 


\title{
Chapter VI
}

\begin{abstract}
High-altitude hypoxia and echocardiographic indices of pulmonary hypertension in male and female chickens at adulthood.
\end{abstract}

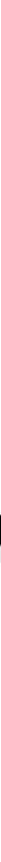

Circ J. 2014;78:1459-64 



\title{
High-Altitude Hypoxia and Echocardiographic Indices of Pulmonary Hypertension in Male and Female Chickens at Adulthood
}

\author{
Carlos E. Salinas, MD; Carlos E. Blanco, MD, PhD; Mercedes Villena, MD; Dino A. Giussani, PhD
}

\begin{abstract}
Background: By combining the chick embryo model with incubation at high altitude $(\mathrm{HA})$, the effects of chronic hypoxia on fetal growth, fetal cardiac and aortic wall remodeling and systemic arterial blood pressure at adulthood were reported. Using non-invasive functional echocardiography, here we investigated the in vivo effects of HA hypoxia on the pulmonary circulation at adulthood in male and female chickens.
\end{abstract}

Methods and Results: Chick embryos were incubated, hatched and raised at sea level (SL) or at HA. At 6 months of age, functional echocardiography was performed and the body and heart weights were taken. Heart weight was heavier in males but not in female HA chickens compared to their same sex SL counterparts. Similarly, male but not female HA chickens had greater in vivo right ventricular wall thickness compared to their same sex SL counterparts. The tricuspid pressure gradient was greatly enhanced in HA male and HA female chickens. However, the increment in the tricuspid pressure gradient was greater in HA males than in HA females. The pulmonary artery diameter was also enhanced in HA males than in SL. males. In contrast, HA did not affect this variable in female chickens.

Conclusions: The data show that chronic hypoxia during development at HA is associated with echocardiocraphic indices of pulmonary hypertension at adulthood in a highly sex-dependent manner. (Circ $J$ 2014; 78: 1459-1464)

Key Words: Cardiovascular disease; Chronic hypoxia; Programming; Pulmonary hypertension

$\mathbf{P}$ ulmonary hypertension continues to be an important clinical problem. ${ }^{1-8}$ Studies of populations at high altitude (HA) have unequivocally reported intrauterine growth restriction (IUGR) and a higher prevalence of pulmonary hypertension, ${ }^{,-17}$ suggesting that a component of these conditions is associated with exposure to chronic hypoxia. However, because most highland populations are also impoverished, the relative contributions of chronic hypoxia or of chronic malnutrition during the fetal and postnatal periods in stunting growth and promoting pulmonary vascular disease during life at altitude remain uncertain.

Similarly, clinical studies at sea level (SL) have reported an association between the IUGR infant and the early development of right ventricular dysfunction and pulmonary hypertension. ${ }^{18-20}$ However, because IUGR in human high-risk pregnancy normally occurs as a result of increased placental vascular impedance with consequent falls in oxygen and nutrient delivery to the baby, the relative contributions of chronic hypoxia or of chronic malnutrition during the fetal period in slowing growth and promoting pulmonary vascular anomalies under these con- ditions, again, remain uncertain.

Experimental studies in animal models, including our own, have used exposure of pregnant mammals to chronic hypobaric or isobaric hypoxia during gestation and have studied the effects on fetal growth and on the cardiovascular system of the offspring in the newborn and adult periods. ${ }^{21-23}$ Studies such as these have reported that chronic fetal hypoxia can program persistent pulmonary hypertension in the newborn and pulmonary hypertension in the adult offspring. ${ }^{24}$ However, because maternal exposure to hypoxia can lead to a significant decrease in maternal food intake, ${ }^{25}$ the extent to which any adverse effects on the pulmonary circulation of the offspring are due to under-nutrition and/or under-oxygenation, once again, remain unclear.

The combination of HA exposure with the use of the chick embryo model permits investigation of the direct effects of HA hypoxia on growth and on cardiovascular development completely independent of alterations in placental function, independent of changes in the maternal physiology and independent of any effects of socioeconomic factors. Previously, we

Received October 29, 2013; revised manuscript received February 11, 2014; accepted February 27, 2014; released online April 16, 2014 Time for primary review: 21 days

Instituto Boliviano de Biología de Altura, Facultad de Medicina, Universidad Mayor de San Andrés, La Paz (C.E.S., M.V.), Bolivia; National Children's Research Centre, Our Lady's Children's Hospital, Crumlin, Dublin (C.E.B.), Ireland; and Department of Physiology, Development and Neuroscience, University of Cambridge, Cambridge (D.A.G.), UK

Mailing address: Dino A. Giussani, PhD, Department of Physiology, Development \& Neuroscience, University of Cambridge, Cambridge CB2 3EG, UK. E-mail: dag26@cam.ac.uk

ISSN-1346-9843 doi:10.1253/circj.CJ-13-1329

All rights are reserved to the Japanese Circulation Society. For permissions, please e-mail: cj@j-circ.or.jp 


\begin{tabular}{|c|c|c|c|c|}
\hline & \multicolumn{2}{|c|}{ Males } & \multicolumn{2}{|c|}{ Females } \\
\hline & SL & HA & SL & HA \\
\hline $\mathrm{pHa}$ & $7.51 \pm 0.03$ & $7.51 \pm 0.01$ & $7.56 \pm 0.04$ & $7.53 \pm 0.03$ \\
\hline $\mathrm{PaCO}_{2}(\mathrm{mmHg})$ & $27.8 \pm 2.2$ & $25.9 \pm 2.1$ & $28.9 \pm 2.4$ & $26.2 \pm 2.1$ \\
\hline $\mathrm{PaO}_{2}(\mathrm{mmHg})$ & $87.1 \pm 3.6$ & $45.4 \pm 3.2^{*}$ & $85.7 \pm 4.1$ & $43.4 \pm 2.9^{*}$ \\
\hline SatHb (\%) & $97.4 \pm 0.4$ & $58.5 \pm 7.2^{*}$ & $97.2 \pm 0.2$ & $60.6 \pm 5.3^{*}$ \\
\hline Htc (\%) & $30.6 \pm 1.1$ & $44.6 \pm 2.1^{*}$ & $27.7 \pm 1.8$ & $46.1 \pm 2.3^{*}$ \\
\hline
\end{tabular}

Values are the mean \pm SEM for arterial $\mathrm{pH}(\mathrm{pHa})$, arterial partial pressure of carbon dioxide $\left(\mathrm{P}_{\mathrm{a}} \mathrm{CO}_{2}\right)$, arterial partial pressure of oxygen $\left(\mathrm{PaO}_{2}\right)$, hemoglobin saturation with oxygen (SatHb) and hematocrit $(\mathrm{Htc})$ in 7 males and 7 female chickens incubated, hatched and raised at sea level (SL) and in 7 male and 7 female chickens incubated, hatched and raised at high altitude (HA). Significant differences $(P<0.05)$ are: ${ }^{*} S L$ vs. HA (Two-way ANOVA + Student-NewmanKeuls post-hoc test).
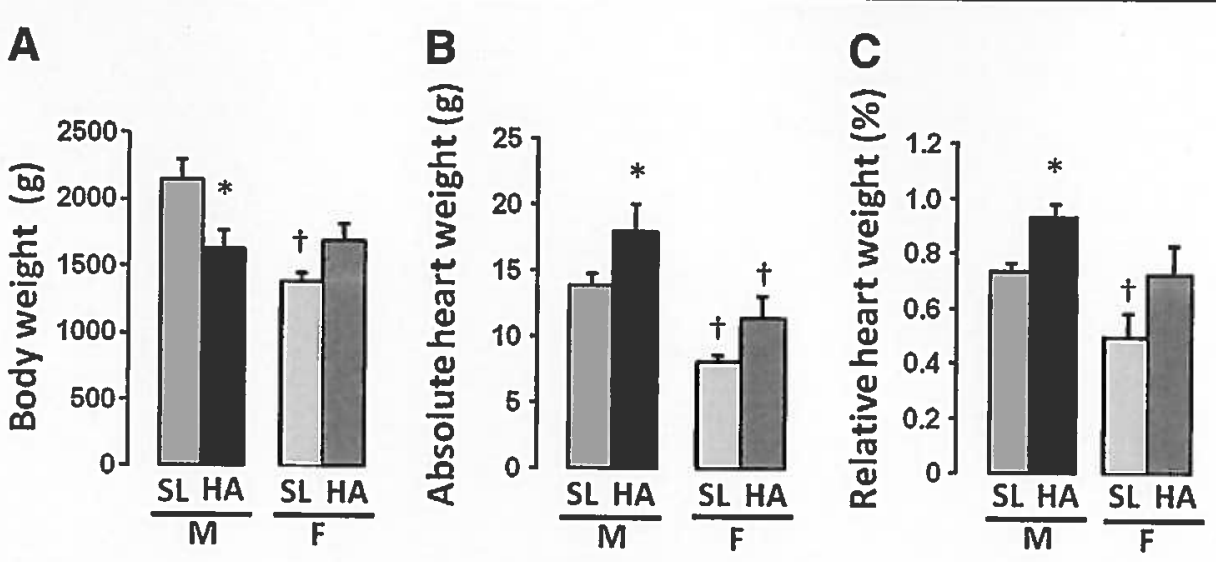

Figure 1. Bodyweight and heart weights in sea-level and high-altitude adult chickens. Values are the mean $\pm S E M$ for bodyweight (A), absolute heart weight (B) and heart weight expressed as a percentage of bodyweight (C) in 7 males $(M)$ and 7 temale (F) chickens incubated, hatched and raised at sea level (SL, light blue and pink, respectively) and in 7 male and 7 female chickens incubated, hatched and raised at high altitude ( $\mathrm{HA}$, dark blue and dark pink, respectively). Significant differences $(P<0.05)$ are: "SL vs. HA, for same sex (sex independent of hypoxia) and tmale vs. female, same altitude (hypoxia independent of sex). A twoway ANOVA + Student-Newman-Keuls post-hoc test.

have reported that incubation of fertilised eggs from SL hens at HA promoted growth restriction, cardiomegaly, cardiac and aortic wall thickening in the chick embryo, and systemic blood pressure dysregulation in the adult chicken. ${ }^{26-28}$ Using functional echocardiography, this study investigated in vivo in real time the effects of HA hypoxia on the pulmonary and systemic circulations in chickens at adulthood. As sexual dimorphic effects on cardiovascular disease are established, ${ }^{29}$ we studied both male and female chickens.

\section{Methods}

All experiments were approved by the local ethics committee of the Bolivian Institute for HA Biology (Consejo Técnico, IBBA, Universidad Mayor de San Andrés, La Paz, Bolivia) and all procedures were performed under the UK Animals (Scientific Procedures) Act 1986.

The study took place in Bolivia, at the HA city of La Paz (HA, 3,600 m, $494 \mathrm{mmHg}, \mathrm{PO}_{2} 100 \mathrm{mmHg}$ ) and the SL city of Santa Cruz (SL, 420 m, $760 \mathrm{mmHg}, \mathrm{PO}_{2} 160 \mathrm{mmHg}$ ). Twentyeight (14 male and 14 female) Black Leghorn chicken embryos were incubated, hatched and raised at SL and twenty-eight (14 males and 14 females) Black Leghorn chicken embryos were incubated, hatched and raised at HA. At 6 months of age (adulthood), in 7 males and 7 females in each group, the femoral artery was catheterised (polyvinyl catheters: i.d. $0.58 \mathrm{~mm}$; o.d. $0.96 \mathrm{~mm}$; Critchly Electrical Products, NSW, Australia) under anaesthesia $(10 \mathrm{mg} / \mathrm{kg}$ Xylazine $2 \%$, Millpledge Pharmaceuticals, $\mathrm{UK}$ and $30 \mathrm{mg} / \mathrm{kg}$ Ketamine, Ketaset, Fort Dodge Animal Health, Iowa, USA, i.m.) and arterial blood samples were taken after 5 days of post-operative recovery for determination of arterial blood gases, acid base status and hematocrit, in duplicate. Another 7 males and 7 females in each group were used for echocardiography studies. These chickens were mildly anaesthetised $(10 \mathrm{mg} / \mathrm{kg}$ Xylazine $2 \%$, Millpledge Pharmaceuticals, UK and $15 \mathrm{mg} / \mathrm{kg}$ Ketamine, Ketaset, Fort Dodge Animal Health, Iowa, USA, i.m.) and placed in a supine position on a heating pad, taking care to minimise body temperature loss. The feathers in the chest region were carefully plucked and echocardiography was performed (Acuson Siemens, Mountain View, CA) using a pediatric probe $7 \mathrm{v} 3 \mathrm{c}(3.5-7 \mathrm{MHz})$, applying standard techniques similar to those described before. ${ }^{20}$ Longitudinal and transverse images were obtained at different levels of the heart in the parasternal long- and short-axis using Mmode bi-dimensional (2D) echocardiography. The thickness of the ventricular walls in real time was measured using the 
A

Right ventricle

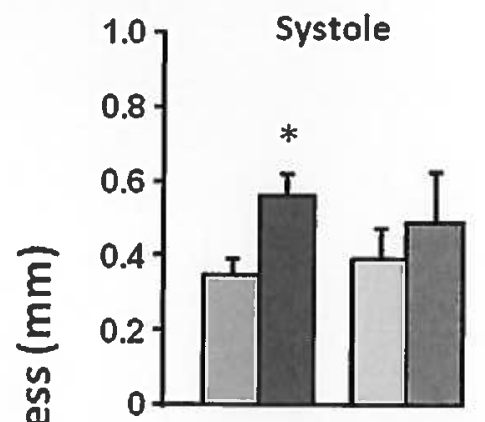

B

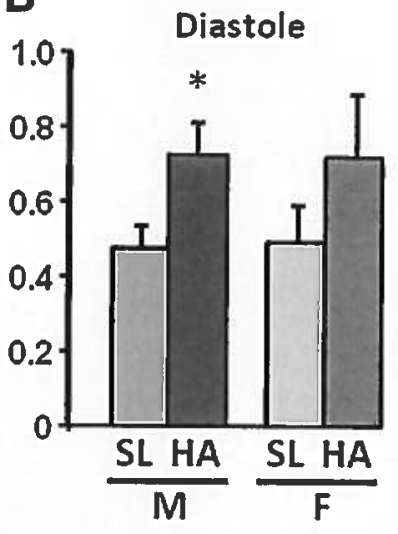

C

\section{Left ventricle}

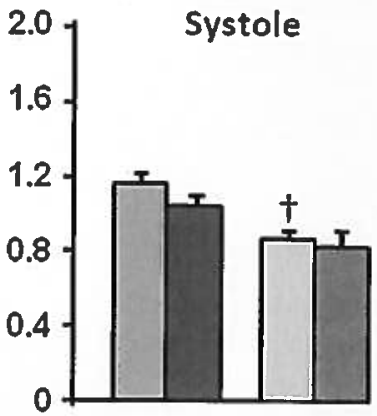

\section{D}

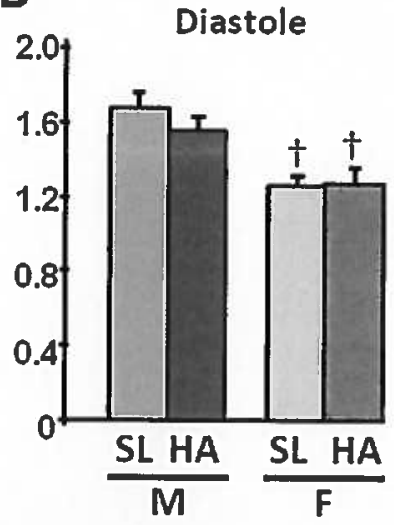

Figure 2. Ventricular wall thickness in sealevel and high-altitude adult chickens. Values are the mean $\pm S E M$ for the wall thickness of the right ( $\mathbf{A}$ and $\mathbf{B})$ and left ( $C$ and $\mathbf{D})$ ventricle during systole and diastole in 7 male (M) and 7 female (F) chickens incubated, hatched and raised at sea level (SL, light blue and pink, respectively) and in 7 male and 7 female chickens incubated, hatched and raised at high altitude (HA, dark blue and dark pink, respectively). Significant differences $(P<0.05)$ are: "SL vs. HA, for same sex (sex independent of hypoxia) and tmale vs. female, same altitude (hypoxia independent of sex). A twoway ANOVA + Student-Newman-Keuls posthoc test. parasternal long-axis view of the heart with the M-mode beam tip just beyond the atrioventricular valves, perpendicular to the long axis of either ventricle. The thickness of the walls of the major vessels was also determined using the parasternal longaxis view of the heart with M-mode. Doppler was used to determine the direction of blood flow and its velocity. In the parasternal long-axis orientation, with B-mode visualization of the pulmonary artery, the pulmonary artery Doppler was established. The peak flow velocity of the trans-tricuspid jet was measured and the pressure gradient between the right ventricle and the right atrium was calculated, as previously described and validated at HA..$^{20}$ The equivalent was determined for the left ventricle. At the end of the experiments, the chicken was humanely killed with an overdose of anaesthetic $(100 \mathrm{mg} / \mathrm{kg}$, Thiopental injection BP, Link Pharmaceuticals Ltd, UK, i.v.). Upon post mortem, the chicken was weighed. The heart was isolated, weighed and frozen in liquid nitrogen.

\section{Statistical Analysis}

All data are expressed as mean \pm SEM. Comparisons between groups were assessed statistically using a 2-way ANOVA with the Student-Newman-Keuls post-hoc test, with altitude and sex as factors (Prism 5, GraphPad Software, Inc). For all comparisons, statistical significance was accepted when $P<0.05$.

\section{Results}

\section{Arterial Blood Gas Status and Hematocrit}

At 6 months, there were no differences in arterial $\mathrm{pH}$ and $\mathrm{pCO}_{2}$ between males and females or between SL and HA. However, $\mathrm{HA}$ male and female chickens had lower arterial $\mathrm{pO}_{2}, \mathrm{SaO}_{2}$ and increased hematocrit compared to SL chickens. Values for $\mathrm{pO}_{2}, \mathrm{SaO}_{2}$ and hematocrit were similarly altered in male and female chickens at HA relative to SL (Table).

\section{Biometry}

At 6 months, bodyweight, absolute heart weight and the heart weight expressed as a percentage of bodyweight were all significantly lower in SL female chickens than SL male chickens (Figures 1A-C). Male but not female chickens at HA were significantly lighter than their same sex SL counterparts (Figure 1A). Similarly, the absolute and relative heart weights were significantly greater only in male but not female HA chickens relative to their same sex SL counterparts (Figures 1A-C).

\section{Echocardiography}

At 6 months, the thickness of the right ventricular wall was similar during systole and diastole in SL male and SL female chickens (Figures 2A,B). However, the thickness of the left ventricular wall was significantly lower during systole and diastole in SL female than in SL male chickens (Figures 2C,D). Male but not female chickens at HA had significantly greater 


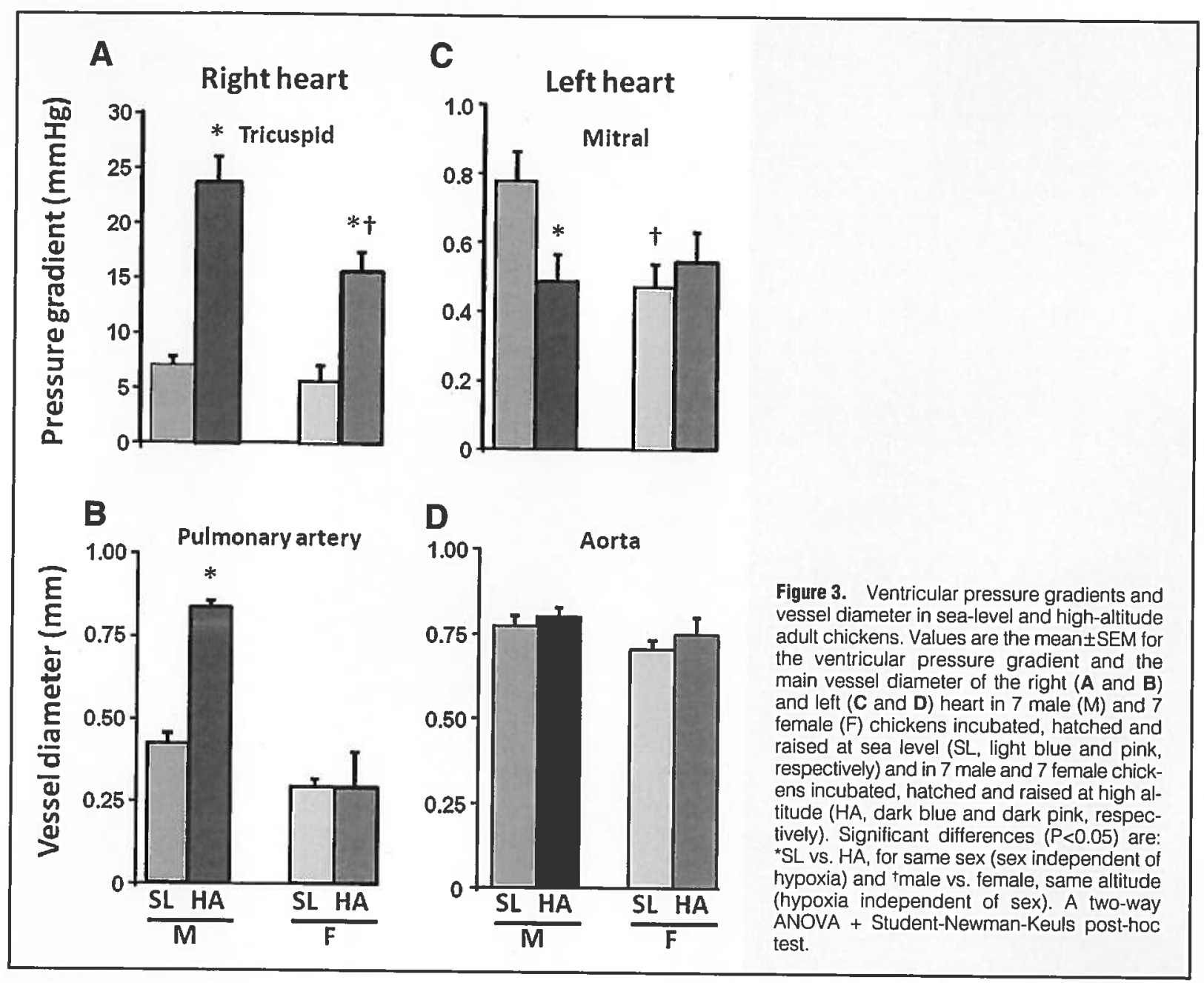

right ventricular wall thickness during systole and diastole than their same sex SL counterparts (Figures 2A,B). HA did not affect the wall thickness of the left ventricle in either males or females (Figures 2C,D).

At 6 months, the tricuspid pressure gradient was greatly enhanced in HA male and HA female chickens relative to their same sex SL counterparts. However, the increment in the tricuspid pressure gradient was significantly greater in HA males than in HA females (Figure 3A). The pulmonary artery diameter was also greatly enhanced in HA male than in SL males. In contrast, HA did not affect the pulmonary artery diameter in female chickens (Figure 3B). Overall, values for the mitral pressure gradient were much lower than values for the tricuspid pressure gradient (Figures 3A,C). The mitral pressure gradient was significantly lower in SL females relative to SL males and in HA males relative to SL males (Figures 3C,D). Neither sex nor HA affected the diameter of the aorta.

\section{Discussion}

Using non-invasive functional echocardiography, data in the present study show that chickens incubated, hatched and raised at HA develop significant indices of pulmonary hypertension at adulthood in a highly sex-dependent manner.

In contrast to the systemic circulation which dilates, the pul- monary vascular bed constricts during hypoxic conditions; $;^{30}$ this is a physiological response, matching pulmonary perfusion to reduced oxygenation. However, excessive or prolonged increases in pulmonary vascular resistance can lead to pathology. Highland residents provide an excellent model to investigate the pathophysiology of the pulmonary vascular bed as they live in an environment of hypobaric hypoxia. Their hearts and pulmonary circulation show alterations that resemble those that occur in clinical conditions associated with alveolar hypoxia and polycythemia, exhibiting pulmonary hypertension and cardiomegaly due to right ventricular hypertrophy. As highlanders lose their capacity for adaptation with advancing age or due to additional risk factors, such as smoking, these findings become exaggerated leading to overt chronic mountain sickness. The expression of pulmonary hypertension and right heart remodelling in highland human and animal residents has been described for many years in a long and rich history of important studies. ${ }^{10-15}$ Although sex differences in the prevalence of pulmonary hypertension at SL have been reported, there is disagreement about whether this is primarily a disease of male or female individuals. ${ }^{31,32}$ In marked contrast, it is a widely held view that the highland female is relatively protected than the highland male against developing pulmonary hypertension during residence at HA. However, this has not been established in the literature. Data are beginning to surface to indicate 
protection against pulmonary hypertension in native highland human residents, such as in the Aymaras, relative to newcomers, and relative protection in Aymara girls relative to Aymara boys. ${ }^{13,33}$ Therefore, the present study advances the literature to report marked protection against echocardiographic indices associated with pulmonary hypertension in adult female chickens relative to male chickens when incubated, hatched and raised at HA. As our study involved chickens, the development of differential indices of pulmonary hypertension in males and females is clearly independent of possible alterations in socioeconomic factors, in alterations in the physiology of the mother animal, or in changes in placental function as may happen in humans, thereby isolating the effect to be due to HA hypoxia. The mechanism underlying protection against high altitudeinduced pulmonary vascular dysfunction in highland natives or in females is not known. However, it might involve differences in the bioavailability of nitric oxide (NO). ${ }^{13,24,35}$ There is considerable evidence highlighting the importance of both pulmonary vascular endothelial and alveolar epithelial NO synthesis in the appropriate regulation of the pulmonary circulation and its adequate response to hypoxia. ${ }^{13}$ Therefore, it is of interest that exhaled NO is much greater in Andean and Tibetan natives than in SL residents, ${ }^{34}$ and that estrogen dilates the pulmonary vascular bed and ameliorates pulmonary hypertension via NO-dependent mechanisms. ${ }^{35}$

In the fields of pulmonary hypertension and of programming of disease, there is increasing interest in establishing answers to 2 unknown questions: whether chronic fetal hypoxia might itself increase susceptibility to developing pulmonary hypertension at adulthood, and whether matching of the pre- and postnatal environments might ameliorate the development of pulmonary hypertension. It has been reported that it is the mismatch between the pre- and post-natal environments that might be more important than adverse intrauterine or post-natal conditions, per se, in rendering the offspring at increased risk of developing disease at adulthood. ${ }^{36}$ In our study, because the environmental condition of hypoxia occurred in ovo as well as post-hatching, the partial contributions of HA hypoxia during the fetal or postnatal periods in triggering pulmonary vascular changes at adulthood cannot be distinguished. However, in the context of the mismatch hypothesis, ${ }^{36}$ the present data are also novel because significant indices of pulmonary vascular dysfunction occurred in adult chickens despite matching of the environment pre- and post-hatching. In the present study, pulmonary vascular anomalies might therefore have developed in response to a double insult; one occurring prior and one after hatching, modeling precisely the continued pre- and post-natal hypoxic environment that HA human populations experience.

Evidence in the literature supporting a primary effect of chronic fetal hypoxia vs. chronic post-natal hypoxia in increasing susceptibility to the onset of pulmonary hypertension in later life is of mixed opinion. Independent evidence of cardiac biventricular hypertrophy and a significant increase in right ventricular wall area and thickness in chick embryos incubated at HA or during chronic isobaric hypoxia, even prior to hatching, ${ }^{27_{r} 37}$ supports a primary effect triggered by chronic hypoxia already during the incubation period, which persists and/or becomes exacerbated by exposure to hypoxia after hatching. Similarly, there have been reports of children resident at HA diagnosed with pulmonary hypertension. ${ }^{38-40}$ Experiments in ovine pregnancy at HA have also reported newborn offspring with basal pulmonary hypertension and an exaggerated increase in pulmonary arterial pressure to a superimposed episode of acute hypoxia. ${ }^{41-46}$ Rueda-Clausen et al. have also reported that chronic hypoxic pregnancy in rodents, followed by post-natal normoxic conditions, leads to pulmonary hypertension in the adult offspring, becoming prominent with aging. ${ }^{24}$ In contrast, there have also been reports in children resident at $\mathrm{HA}$ with no evidence of pulmonary hypertension, when socioeconomic factors were accounted for. ${ }^{47}$ Similarly, experimental studies in newborn rats and guinea pigs exposed to chronic hypoxia in utero have reported no morphological evidence of pulmonary hypertension. ${ }^{48-50)}$ Finally, no evidence of early endothelial dysfunction was reported in small pulmonary arteries of fast-growing broilers raised in normoxia following incubation under hypoxic conditions. ${ }^{51}$ Clearly, further insight into this debate could be obtained by exploiting the combination of the chick embryo model and HA exposure, but with a cross-over study design; by investigating adult offspring (pre- and post-puberty) incubated at HA but raised post-hatching at SL and vice versa. Although logistically rather more difficult, this is clearly an obvious extension of the present work and a path for future investigation.

In the present study, there are 2 additional findings that deserve some attention. First, the tricuspid pressure gradient was greatly enhanced in highland chickens. However, the increment in the tricuspid pressure gradient was significantly greater in highland males than in highland females. In contrast, the pulmonary artery diameter was also greatly enhanced in highland chickens, but only in males. HA did not affect the pulmonary artery diameter in female chickens. Second, the mitral pressure gradient was significantly decreased in highland males relative to SL males. The reasons for the dissociation between an effect of HA on the tricuspid pressure gradient but not on the pulmonary artery vessel diameter in female chickens are unclear. However, it might indicate the existence of a threshold tricuspid pressure gradient above which remodelling of the pulmonary vessel wall is triggered, as in highland male chickens. The lower mitral pressure gradient between highland vs. SL males might indicate relative systemic arterial hypotension in highland males. Of interest, a recent study by our group has reported that HA chickens had significantly lower arterial blood pressure than SL chickens, when measured in chronically instrumented animals in vivo. However, this effect was independent of the sex of the animal.28

In conclusion, by combining the chick embryo model with incubation at $\mathrm{HA}$, we have investigated the in vivo effects of chronic hypoxia on the pulmonary system at adulthood, and show that pre- and post-hatching development at HA markedly enhances established echocardiographic indices of pulmonary hypertension at adulthood in a highly sex-specific manner.

\section{Acknowledgments}

This work was supported by The British Heart Foundation and The Royal Society. Dino A. Giussani is the Professor of Cardiovascular Physiology \& Medicine at the Department of Physiology Development \& Neuroscience at the University of Cambridge, Professorial Fellow and Director of Studies in Medicine at Gonville \& Caius College, a Lister Institute Fellow and a Royal Society Wolfson Research Merit Award Holder.

We thank Dr Diva Bellido, Dr Wilma Tellez, Mr Armando Rodriguez, Mrs Martha Aguilar, Mrs Loyola Riveros, Mr Wilmar Velasquez and Mr Didi Maquera at IBBA, La Paz, and Dr Regien Biesma, Dr Ginella, Dr Nioshi and Dr Roca for their help with these studies at CENETROP, Santa Cruz.

\section{Conflict of Interest}

The authors can confirm that they hold no conflict of interest.

\section{References}

1. Inaba $T$, Yao A, Nakao T, Hatano M, Maki H, Imamura T, et al. Volumetric and functional assessment of ventricles in pulmonary hypertension on 3-dimensional echocardiography. Circ J 2013; 77: 198 - 
206.

2. Katsuragi S, Yamanaka K, Neki R, Kamiya C, Sasaki Y, Osato K, et al. Maternal outcome in pregnancy complicated with pulmonary arterial hypertension. Circ J 2012; 76: 2249-2254.

3. Kopeć G, Tyrka A, Miszalski-Jamka T, Sobień M, Waligóra $M$, Brózda M, et al. Electrocardiogram for the diagnosis of right ventricular hypertrophy and dilation in idiopathic pulmonary arterial hypertension. Circ $J$ 2012; 76: 1744-1749.

4. Yamada Y, Okuda S, Kataoka M, Tanimoto A, Tamura Y, Abe T, et al. Prognostic value of cardiac magnetic resonance imaging for idiopathic pulmonary arterial hypertension before initiating intravenous prostacyclin therapy. Circ J 2012; 76: 1737-1743.

5. Ogawa A, Miyaji K, Yamadori I, Shinno Y, Miura A, Kusano KF, et al. Safety and efficacy of epoprostenol therapy in pulmonary venoocclusive disease and pulmonary capillary hemangiomatosis. Circ $J$ 2012; 76: 1729-1736

6. Taguchi H, Kataoka M, Yanagisawa R, Kawakami T, Tamura Y, Fukuda $\mathrm{K}$, et al. Platelet level as a new prognostic factor for idiopathic pulmonary arterial hypertension in the era of combination therapy. Circ J 2012; 76: 1494-1500.

7. Chida A, Shintani M, Nakayama T, Furutani Y, Hayama E, Inai K, et al. Missense mutations of the BMPRIB (ALK6) gene in childhood idiopathic pulmonary arterial hypertension. Circ J 2012; 76: 1501 1508.

8. Yanagisawa $R$, Kataoka $M$, Taguchi $H$, Kawakami T, Tamura $Y$, Fukuda $\mathrm{K}$, et al. Impact of first-line sildenafil monotreatment for pulmonary arterial hypertension. Circ J 2012; 76: 1245-1252.

9. Moore LG, Charles SM, Julian CG. Humans at high altitude: Hypoxia and fetal growth. Respir Physiol Neurobiol 201 1; 178: 181 - 190.

10. Hurtado A. Chronic mountain sickness. JAMA 1942; 120: 1278-1282

11. Monge MC, Monge CC. High altitude diseases: Mechanism and management. Springfield (III): Charles C. Thomas, 1966.

12. Morrell NW, Sarybaev AS, Alikhan A, Mirrakhimov MM, Aldashev AA. ACE genotype and risk of high altitude pulmonary hypertension in Kyrghyz highlanders. Lancet 1999; 353: 814.

13. Scherrer U, Turini P, Thalmann S, Hutter D, Salinas Salmón C, Stuber $\mathrm{T}$, et al. Pulmonary hypertension in high-altitude dwellers: Novel mechanisms, unsuspected predisposing factors. Adv Exp Med Biol 2006; 588: $277-291$.

14. Peñaloza D, Arias-Stella J. The heart and pulmonary circulation at high altitudes: Healthy highlanders and chronic mountain sickness. Circulation 2007; 115: 1132-1146.

15. West JB. High-altitude medicine. Am J Respir Crit Care Med 2012; 186: 1229 - 1237 .

16. Haas JD, Frongillo EF, Stepcik C, Beard J, Hurtado L. Altitude, ethnic and sex differences in birthweight and length in Bolivia. Httm Biol 1980; 52: 459-477.

17. Giussani DA, Phillips PS, Anstee S, Barker DJ. Effects of altitude vs. economic status on birth weight and body shape at birth. Ped Res 2001; 49: 490-494.

18. Danhaive O, Margossian R, Geva T, Kourembanas S. Pulmonary hypertension and right ventricular dysfunction in growth-restricted, extremely low birth weight neonates. J Perinatol 2005; 25: 495-499.

19. Rosenberg A. The IUGR newborn. Semin Perinatol 2008; 32: 219 224.

20. Jayet PY, Rimoldi SF, Stuber T, Salmòn CS, Hutter D, Rexhaj E, et al. Pulmonary and systemic vascular dysfunction in young offspring of mothers with preeclampsia. Circulation 2010; 122: 488-494.

21. Patterson AJ, Zhang L. Hypoxia and fetal heart development. Curr Mol Med 2010; 10: 653-666.

22. Morton JS, Rueda-Clausen CF, Davidge ST. Mechanisms of endothelium-dependent vasodilation in male and female, young and aged offspring borm growth restricted. Am J Physiol Regul Integr Comp Physiol 2010; 298: R930-R938.

23. Giussani DA, Camm EJ, Niu Y, Richter HG, Blanco CE, Gottschalk $\mathrm{R}$, et al. Developmental programming of cardiovascular dysfunction by prenatal hypoxia and oxidative stress. PLOS One 2012; 7: e31017, doi: $10.1371 /$ journal.pone.0031017 [Epub ahead of print].

24. Rueda-Clausen CF, Morton JS, Davidge ST. Effects of hypoxia-induced intrauterine growth restriction on cardiopulmonary structure and function during adulthood. Cardiovasc Res 2009; 81: 713-722.

25. de Grauw TJ, Myers RE, Scott WJ. Fetal growth retardation in rats from different levels of hypoxia. Biol Neonate 1986; 49: 85-89.

26. Giussani DA, Salinas CE, Villena M, Blanco CE. The role of oxygen in prenatal growth: Studies in the chick embryo. J Physiol 2007; 585: 911-917.

27. Salinas CE, Blanco CE, Villena M, Camm EJ, Tuckett JD, Weerakkody RA, et al. Developmental origin of cardiac and vascular disease in chick embryos incubated at high attitude. J DOHaD 2010; 1: 60-66.
28. Herrera EA, Salinas CE, Blanco CE, Villena M, Giussani DA. High altitude hypoxia and blood pressure dysregulation in adult chickens. JDOHaD 2013; 4: 69-76.

29. Morita N, Okita K. Is gender a factor in the reduction of cardiovascular risks with exercise training? Circ $J 2013$; 77: 646-651.

30. Sylvester JT, Shimoda LA, Aaronson PI, Ward JP. Hypoxic pulmo nary vasoconstriction. Physiol Rev 2012;92: 367-520.

31. Shapiro S, Traiger GL, Turner M, McGoon MD, Wason P, Barst RJ. Sex differences in the diagnosis, treatment, and outcome of patients with pulmonary arterial hypertension enrolled in the registry to evaluate early and long-term pulmonary arterial hypertension disease man agement. Chest 2012; 141: 363-373.

32. Jacobs W, van de Veerdonk MC, Trip P, de Man F, Heymans MW, Marcus JT, et al. The right ventricle explains sex differences in survival in idiopathic pulmonary arterial hypertension. Chest 2013 December 5, doi: 10.1378/chest.13-1291 [Epub ahead of print].

33. Turini P, Allemann $Y$, Sartori C, Hutter D, Thalmann S, Salinas C, et al. Protective effects of female sex hormones against pulmonary hypertension in Bolivian high altitude natives. Circulation 2003; 111: B54.

34. Beall CM, Laskowski D, Strohl KP, Soria R, Villena M, Vargas E, et al. Pulmonary nitric oxide in mountain dwellers. Nature $2001 ; 414$ 411-412.

35. Yuan P, Wu WH, Gao L, Zheng ZQ, Liu D, Mei HY, et al. Oestradiol ameliorates monocrotaline pulmonary hypertension via NO, prostacyclin and endothelin-1 pathways. Eur Respir J 2013;41: 1116-1125.

36. Gluckman PD, Hanson MA. Mismatch: The Lifestyle Diseases Time Bomb. Melbourne: Oxford University Press, 2006.

37. Villamor E, Kessels CG, Ruijtenbeek K, van Suylen RJ, Belik J, de Mey JG, et al. Chronic in ovo hypoxia decreases pulmonary arterial contractile reactivity and induces biventricular cardiac enlargement in the chicken embryo. Am J Physiol Regul Integr Comp Ploysiol 2004; 287: R642-R651.

38. Niermeyer $S$. Cardiopulmonary transition in the high altitude infant High Alt Med Biol 2003; 4: 225-239.

39. Peñaloza D, Arias-Stella J, Sime F, Recavarren S, Marticorena E. The heart and pulmonary circulation in children at high altitudes: Physiological, anatomical, and clinical observations. Pediatrics 1964; 34: $568-582$.

40. Peñaloza D, Sime F, Ruiz L. Pulmonary hemodynamics in children living at high altitudes. High Alt Med Biol 2008; 9: 199-207.

41. Llanos AJ, Ebensperger G, Herrera EA, Reyes RV, Pulgar VM, SerónFerré $\mathrm{M}$, et al. Fetal and postnatal pulmonary circulation in the Alto Andino. Placenta 2011; 32(Suppl 2): S100-S103.

42. Herrera EA, Riquelme RA, Ebensperger G, Reyes RV, Ulloa CE Cabello G, et al. Long-term exposure to high-altitude chronic hypoxia during gestation induces neonatal pulmonary hypertension at sea level. Am J Physiol Regul lntegr Comp Physiol 2010; 299: R1676-R1684.

43. Herrera EA, Ebensperger G, Krause BJ, Riquelme RA, Reyes RV, Capetillo $M$, et al. Sildenafil reverses hypoxic pulmonary hypertension in highland and lowland newborn sheep. Pediatr Res 2008; 63: $169-175$.

44. Herrera EA, Reyes RV, Giussani DA, Riquelme RA, Sanhueza EM Ebensperger G, et al. Carbon monoxide: A novel pulmonary artery vasodilator in neonatal llamas of the Andean altiplano. Cardiovasc Res 2008; 77: 197-201.

45. Llanos AJ, Riquelme RA, Herrera EA, Ebensperger G, Krause B, Reyes RV, et al. Evolving in thin air--lessons from the llama fetus in the altiplano. Respir Physiol Neurobiol 2007; 158: 298-306.

46. Herrera EA, Pulgar VM, Riquelme RA, Sanhueza EM, Reyes RV Ebensperger G, et al. High-altitude chronic hypoxia during gestation and after birth modifies cardiovascular responses in newbom sheep. Am J Physiol Regul Integr Comp Physiol 2007; 292: R2234-R2240.

47. Huicho L, Niermeyer S. Cardiopulmonary pathology among children resident at high altitude in Tintaya, Peru: A cross-sectional study. High Alt Med Biol 2006; 7: 168-179.

48. Goldberg SJ, Levy RA, Siassi B, Betten J. The effects of materna hypoxia and hyperoxia upon the neonatal pulmonary vasculature. $\mathrm{Pe}$ diatrics 1971 ; 48: 528-533.

49. Geggel RL, Aronovitz MJ, Reid LM. Effects of chronic in utero hypoxemia on rat neonatal pulmonary arterial structure. J Pediatr 1986 ; 108: $756-759$.

50. Murphy JD, Aronovitz MJ, Reid LM. Effects of chronic in utero hypoxia on the pulmonary vasculature of the newborn guinea pig. $\mathrm{Pe}$ diatr Res 1986; 20: 292-295.

51. Zoer B, Kessels L, Vereijken A, De Mey JG, Bruggeman V, Decuypere $E$, et al. Effects of prenatal hypoxia on pulmonary vascular reactivity in chickens prone to pulmonary hypertension. J Physiol Pharmacol 2009; 60: $119-130$. 


\section{Chapter VII}

\section{General discussion}

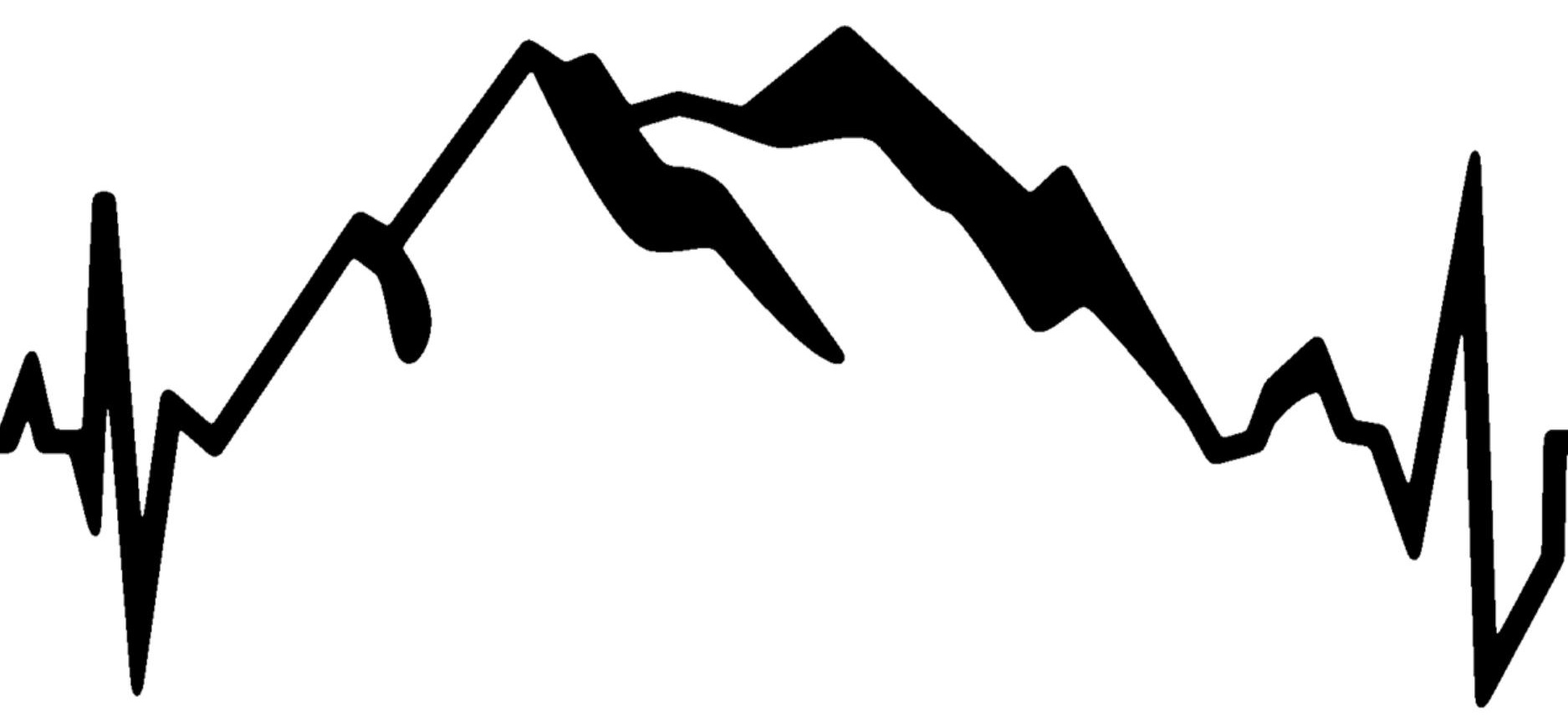

Adapted from

Itani N, Salinas CE, Villena M, Skeffington KL, Beck C, Villamor $\mathrm{E}$, Blanco CE, Giussani DA. The highs and lows of programmed cardiovascular disease by developmental hypoxia: studies in the chicken embryo.

J Physiol. 2017 [Epub ahead of print] 

It is now established that adverse conditions during pregnancy can trigger a fetal origin of cardiovascular dysfunction and/or increase the risk of heart disease in later life. Sub-optimal environmental conditions during early life that may promote the development of cardiovascular dysfunction in the offspring include alterations in fetal oxygenation and nutrition as well as fetal exposure to stress hormones, such as glucocorticoids. There has been growing interest in identifying the partial contributions of each of these stressors to programming of cardiovascular dysfunction. However, in humans and in many animal models this is difficult, as the challenges cannot be disentangled. By using the chicken embryo as an animal model, science has been able to circumvent a number of problems. In contrast to mammals, in the chicken embryo the effects on the developing cardiovascular system of changes in oxygenation, nutrition or stress hormones can be isolated and determined directly, independent of changes in the maternal or placental physiology. In this review, we summarise the findings of the present thesis and of other studies that have exploited the chicken embryo model to determine the effects on prenatal growth, cardiovascular development and pituitary-adrenal function of isolated chronic developmental hypoxia.

\section{Chronic hypoxia and fetal growth: Studies in the chicken embryo}

Incubation of fertilised eggs laid by sea level hens at high altitude $(3600 \mathrm{~m})$ reduced fetal $\mathrm{PO}_{2}$, increased haematocrit and reduced embryonic weight by $45 \%$ at the end of incubation period (Chapter II, Chapter III, Figure 1A). Importantly, the reduction in embryonic weight persisted when it was expressed relative to the egg mass at the start of the incubation period, to account for very real differences in egg size laid by sea level hens compared to high altitude hens (Figure 1B). Incubation of eggs laid by high altitude hens at high altitude also led to significant fetal growth restriction, although the change was smaller (by 22.2\%) compared to the effect of high altitude on chicken embryos of sea level hens. This protection on prenatal growth by prolonged residence at high altitude has also been reported for human Andean populations (Giussani et al., 2001; Moore et al., 2004; Soria et al., 2013), coining the effect as the Andean curse on the conquistadors (Giussani, 2007). Further data revealed that high-altitude induced growth restriction of fertilised eggs laid by sea level hens was prevented by supplementing the incubator with oxygen at pressures to equate sea level conditions (chapter II). Importantly, chicken embryos incubated at high altitude were asymmetrically growth restricted (Figure 1C), with a marked 
increase in the brain weight and head diameter relative to the embryonic body mass (chapters II and III). It is well established that the asymmetric growth restriction is a consequence of fetal blood flow redistribution in response to chronic hypoxia; commonly referred to as the fetal brain sparing effect (Giussani \& Davidge, 2013; Allison et al., 2016; Giussani, 2016). This fetal phenotypic response to chronic fetal hypoxia is well conserved across species including the human, sheep and rat (Mulder et al., 1998; Lang et al., 2000; Ruijtenbeek et al., 2000; Giussani et al., 2001; Fowden et al., 2006; Giussani et al., 2007; Camm et al., 2010), thereby supporting the chicken embryo as an appropriate and comparable animal model to study the consequences of chronic developmental hypoxia on asymmetric growth restriction prior to hatching.

The significant induction of embryonic growth restriction by developmental hypoxia has also been reported by us and others using incubation of chicken embryos under isobaric (sea level atmospheric pressure) hypoxic conditions (Figure 1 and Table 1, Ruijtenbeek et al., 2000; Dzialowski et al., 2002; Miller et al., 2002; Rouwet et al., 2002; Villamor et al., 2004; Sharma et al., 2006; Giussani et al., 2007; Wei et al., 2007; Lindgren \& Altimiras, 2009; Van der Sterren et al., 2009; Zoer et al., 2009; Salinas et al., 2010; Zoer et al., 2010a; Lindgren \& Altimiras, 2011; Lindgren et al., 2011; Sahan et al., 2011; Moonen et al., 2012; Itani et al., 2016b). Varying magnitudes of the reduction in embryo weight in these studies may reflect differences in the humidity, temperature, the length and timing of hypoxia as well as the breed of chicken used. However, combined, past studies describing the significant effect of incubation of fertilised eggs under hypobaric (lower than normal atmospheric pressure) or isobaric hypoxic conditions in slowing embryonic growth add robust evidence to the literature to strongly support a role for isolated fetal hypoxia in mediating fetal growth restriction. 

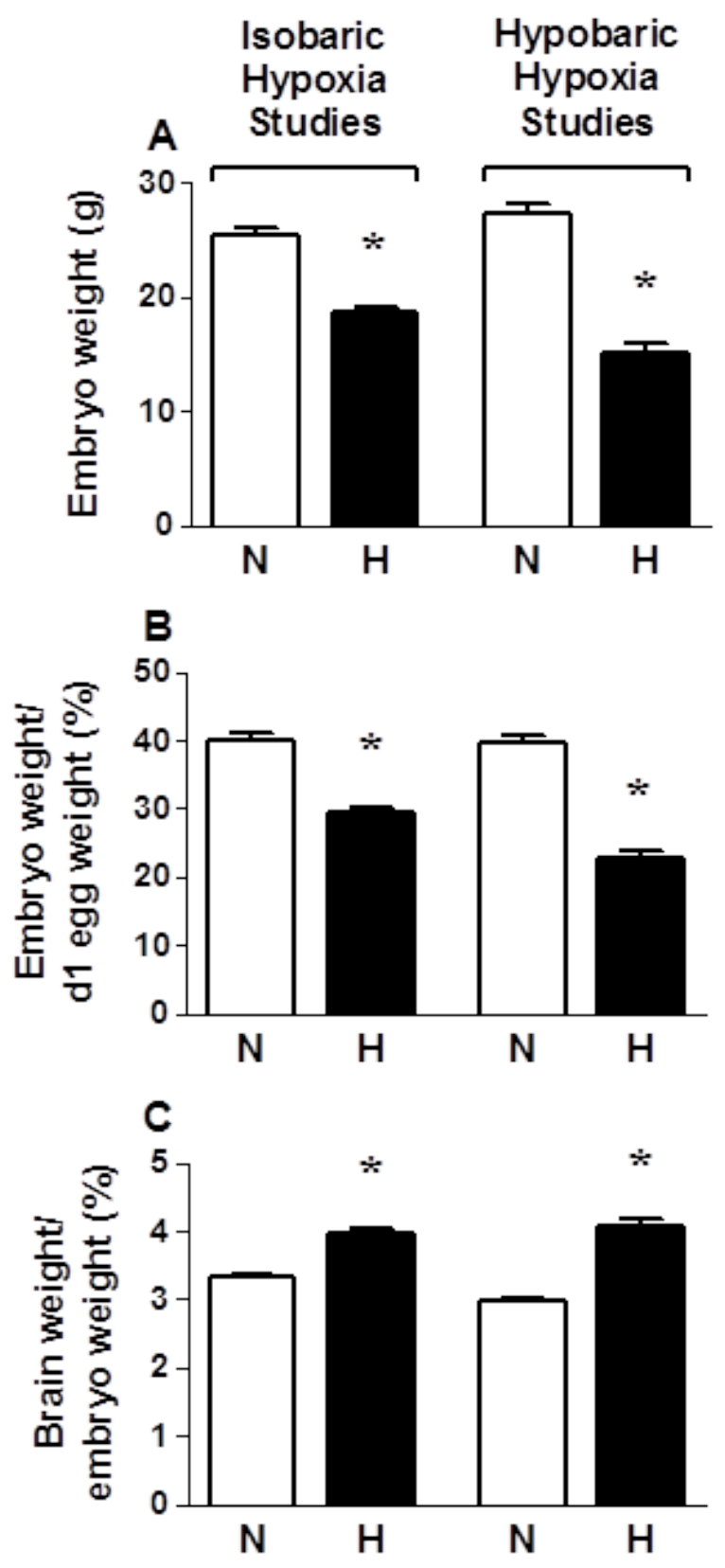

Figure 1. Fetal growth in the chicken embryo at day 19-20 of incubation. Values are mean \pm S.E.M. at day 19-20 of absolute embryo weight (A), relative embryo weight (B), and brain weight relative to body weight $(C)$ of chicken embryos incubated in either normoxia $(N, n=21)$ or hypoxia $(\mathrm{H}, \mathrm{n}=20)$ for isobaric hypoxia studies, and normoxia at sea level $(\mathrm{N}$, $\mathrm{n}=31)$ or hypoxia at high altitude $(\mathrm{H}, \mathrm{n}=16)$ for hypobaric hypoxia studies. *Significantly $(\mathrm{P}<0.05)$ different from corresponding control. Data adapted from chapter II; chapter III; Itani et al. (2016a) and Itani et al. (2016b). 


\begin{tabular}{|c|c|c|c|c|c|c|c|c|c|c|}
\hline \multirow[t]{2}{*}{ Breed } & \multirow[t]{2}{*}{ Study design } & \multirow{2}{*}{$\begin{array}{l}\text { Temp } \\
\left({ }^{\circ} \mathrm{C}\right)\end{array}$} & \multirow{2}{*}{$\begin{array}{l}\text { Humidity } \\
(\%)\end{array}$} & \multicolumn{2}{|c|}{ Oxygen (\%) } & \multirow{2}{*}{$\begin{array}{l}\text { Body } \\
\text { weight } \\
\text { measured } \\
\text { on }\end{array}$} & \multicolumn{2}{|c|}{ Body weight (g) } & \multirow{2}{*}{$\begin{array}{l}\text { Difference } \\
\text { (\%) }\end{array}$} & \multirow[t]{2}{*}{ Reference } \\
\hline & & & & Control & Hypoxia & & Control & Hypoxia & & \\
\hline Black Leghorn & Hypobaric hypoxia & 38 & 60 & 20 & 13.7 & $\mathrm{~d} 20$ & $28 \pm 1$ & $15 \pm 1$ & 46.4 & (Salinas et al., 2010) \\
\hline Broiler Ross 308 & Hypobaric hypoxia & 37.5 & 55 & 20.7 & 18.5 & $\mathrm{~d} 18$ & $48.1 \pm 0.7$ & $46.0 \pm 0.6$ & 4 & (Sahan et al., 2011) \\
\hline Bovans Brown & Isobaric hypoxia & 37.9 & 45 & 21 & 14 & $\mathrm{~d} 19$ & $25.2 \pm 0.9$ & $19.4 \pm 0.5$ & 23 & (Itani et al., 2016b) \\
\hline White Leghorn & Isobaric hypoxia from day 6 & 37.8 & 45 & 21 & 15 & $\mathrm{~d} 19$ & $29.9 \pm 5.6$ & $25.3 \pm 5.5 \mathrm{SD}$ & 15.4 & (Van der Sterren et al., 2009) \\
\hline White Leghorn & Isobaric hypoxia from day 6 & 38 & 60 & 21 & 15 & $\mathrm{~d} 19$ & $26.6 \pm 0.7$ & $22.4 \pm 0.5$ & 15.8 & (Villamor et al., 2004) \\
\hline White Leghorn & Isobaric hypoxia & 37.8 & 60 & 21 & 15 & $\mathrm{~d} 19$ & $28.0 \pm 2.9 \mathrm{SD}$ & $24.8 \pm 1.9 \mathrm{SD}$ & 11.4 & (Moonen et al., 2012) \\
\hline White Leghorn & Isobaric hypoxia & 37.8 & 45 & 21 & 15 & $\mathrm{~d} 19$ & $28.3 \pm 0.4 \mathrm{SD}$ & $23.7 \pm 0.6 \mathrm{SD}$ & 16.3 & (Zoer et al., 2010a) \\
\hline White Leghorn & Isobaric hypoxia & 37 & 60 & 21 & 15 & $\mathrm{~d} 19$ & $24.7 \pm 0.4$ & $21.9 \pm 0.5$ & 11.3 & (Rouwet et al., 2002) \\
\hline White Leghorn & Isobaric hypoxia & 38 & $60-70$ & 21 & 15 & d3.5 & $0.038 \pm 0.002$ & $0.033 \pm 0.001$ & 13.2 & (Sharma et al., 2006) \\
\hline White Leghorn & Isobaric hypoxia from day 10 & 38.5 & $50-60$ & 21 & 14 & $\mathrm{~d} 15$ & $12.2 \pm 1.7 \mathrm{SD}$ & $10.8 \pm 1.6 \mathrm{SD}$ & 10.8 & (Miller et al., 2002) \\
\hline White Leghorn & $\begin{array}{l}\text { Isobaric hypoxia between day } \\
6-12\end{array}$ & 37.5 & $75-95$ & 21 & 15 & $\mathrm{~d} 18$ & $23.4 \pm 0.5$ & $20.7 \pm 0.5$ & 11.5 & (Dzialowski et al., 2002) \\
\hline White Leghorn & $\begin{array}{l}\text { Isobaric hypoxia between day } \\
12-18\end{array}$ & 37.5 & $75-95$ & 21 & 15 & $\mathrm{~d} 18$ & $23.4 \pm 0.5$ & $19.7 \pm 0.5$ & 15.8 & (Dzialowski et al., 2002) \\
\hline White Leghorn & Isobaric hypoxia from day 6 & 37.8 & 45 & 21 & 15 & $\mathrm{~d} 19$ & $29.2 \pm 0.3 \mathrm{SD}$ & $25.9 \pm 0.4 \mathrm{SD}$ & 11.3 & (Zoer et al., 2009) \\
\hline Broiler & Isobaric hypoxia from day 6 & 37.8 & 45 & 21 & 15 & $\mathrm{~d} 19$ & $31.4 \pm 0.6 \mathrm{SD}$ & $25.6 \pm 0.5 \mathrm{SD}$ & 18.5 & (Zoer et al., 2009) \\
\hline Broiler Ross 308 & Isobaric hypoxia & 37.8 & 45 & 20.95 & 14 & $\mathrm{~d} 19$ & $31.7 \pm 3.2 \mathrm{SD}$ & $21.5 \pm 2.8 \mathrm{SD}$ & 32.2 & (Lindgren \& Altimiras, 2009) \\
\hline Broiler Ross 308 & Isobaric hypoxia & 37.8 & 45 & 21 & 14 & $\mathrm{~d} 19$ & $34.8 \pm 0.5$ & $25.7 \pm 0.6$ & 26.1 & (Lindgren et al., 2011) \\
\hline
\end{tabular}

Table 1. Studies showing a reduction in chicken embryo weight following exposure to chronic hypoxia. Body weight data are mean \pm S.E.M unless otherwise stated. SD, standard deviation. 


\section{Chronic hypoxia and the fetal HPA axis: Studies in the chicken embryo}

It is well established that the hypothalamo-pituitary-adrenal (HPA) axis is functional long before hatching in the chicken embryo (Woods et al., 1971; Wise \& Frye, 1973; Kalliecharan \& Hall, 1974; Jenkins \& Porter, 2004). Consequently, possible effects of hypoxia on the developing HPA axis can be isolated using this model. Within the few studies addressing HPA function in the chronically hypoxic chicken embryo, we reported (chapter IV) that incubation at high altitude of fertilised eggs laid by sea level hens significantly increased plasma ACTH concentrations by embryonic day 20, while circulating levels of corticosterone in these embryos were markedly reduced compared to chicken embryos of sea level hens incubated at sea level. In addition, incubation at high altitude of eggs laid by high altitude hens produced similar changes in the levels of fetal plasma ACTH and corticosterone. Moreover, supplementing sea level eggs with oxygen during incubation at high altitude completely prevented the observed changes in ACTH and corticosterone production, thereby supporting hypoxia rather than hypobaria in inducing the adrenocortical blunting. Correlation analysis revealed that the levels of plasma ACTH and corticosterone were positively related in sea level embryos incubated at sea level or at high altitude with oxygen supplementation. However, this correlation was no longer apparent in all other groups. Therefore, data in this study support previous human and mammalian studies reporting dissociated HPA activities in development complicated by chronic fetal hypoxia (Challis et al., 1989; Hooper et al., 1990; Harvey et al., 1993; Murotsuki et al., 1996; Stratford \& Hooper, 1997). Importantly, the chicken embryo data showed that the plasma corticosterone-ACTH ratio was positively correlated to embryonic body weight and to chorioallantoic blood $\mathrm{PO}_{2}$, providing further evidence for a direct relationship between changes in HPA activity and changes in oxygenation during embryonic development (chapter IV).

In line with our findings in the chicken embryo (chapter IV), a study using the sheep fetus demonstrated that development at high altitude promoted the enhanced processing of the precursor proopiomelanocortin into ACTH (Myers et al., 2005). In addition, ACTH receptor expression and enzymatic capacity to synthesise cortisol were both reduced in the fetal sheep adrenal glands in the same experimental model (Myers et al., 2005). In subsequent studies, Myers and Ducsay proposed that nitric oxide (NO) and leptin could be potential mediators of the inhibition of cortisol 
synthesis in the adrenal glands of fetal sheep exposed to long-term high altitude hypoxia (Monau et al., 2009; Myers \& Ducsay, 2012). The authors suggested that leptin and NO limit the capacity of elevated fetal plasma ACTH levels to stimulate cortisol production in the chronically hypoxic fetus. In sheep, this prevents the premature induction of labour while allowing for the maintenance of the normal prepartum surge in fetal plasma cortisol that occurs close to term, which is essential for appropriate fetal maturation.

In addition to adrenocortical blunting, high altitude incubation of chicken embryos from hens native to sea level or to high altitude induced a significant increase in adrenal concentrations of both adrenaline and noradrenaline, and oxygen supplementation during high altitude incubation prevented this (chapter IV). Chronic hypoxia-induced sensitisation of the sympatho-adrenal medullary system has been reported in a range of animal models including the sheep, llama, rat and chicken (Roigas et al., 1996; Simonetta et al., 1997; Ruijtenbeek et al., 2000; Gardner et al., 2002; Llanos et al., 2003; Lindgren \& Altimiras, 2013). For instance, in the chicken embryo, chronic hypoxia sensitises cardiac $\beta$-adrenergic receptors (Lindgren \& Altimiras, 2009, 2013). In addition, chronically hypoxic chicken embryos have increased noradrenaline levels and enhanced sympathetic innervation in the peripheral vasculature (Ruijtenbeek et al., 2000). Importantly, there was a strong negative correlation between arterial $\mathrm{PO}_{2}$ and adrenal catecholamine content in chicken embryos incubated at sea level and high altitude (chapter IV). These data therefore not only support a similar relationship between plasma noradrenaline levels and $\mathrm{PO}_{2}$ reported in fetal sheep (Simonetta et al., 1997), but provide further evidence for a direct effect of isolated developmental hypoxia on the reactivity of the sympathoadrenomedullary system.

\section{Chronic fetal hypoxia and programmed cardiovascular risk: Studies in the chicken embryo and in the adult bird}

It is widely established that adverse conditions during development not only promote IUGR and alterations in the HPA axis, but they also induce early origins of cardiovascular remodelling and dysfunction (see Gluckman et al., 2008; Giussani \& Davidge, 2013). In humans, IUGR pregnancy is associated with ventricular hypertrophy, and increased aortic stiffness and thickness in the offspring before and after birth (Veille et al., 1993; Skilton et al., 2005; Akira \& Yoshiyuki, 2006; Cosmi et al., 2009). Studies in rodent animal models have provided evidence to 
support that the human IUGR-induced programming of cardiovascular remodelling is at least partly due to suspected chronic fetal hypoxia. For instance, hypoxic pregnancy induces similar thickening of the aortic wall and aortic stiffening in the rodent as well as ovine fetus (Williams et al., 2005a; Camm et al., 2010; Thompson et al., 2011; Giussani et al., 2012). Studies by Zhang and colleagues have also reported that hypoxic pregnancy in rodents leads to cardiac remodelling in the offspring (Bae et al., 2003; Tong \& Zhang, 2012). Aortic hypertrophy often precedes the clinical manifestation of hypertension, atherosclerosis and coronary heart disease (Arnett et al., 1994). Consequently, the remodelling of the heart and major vessels early in life due to suspected chronic fetal hypoxia may increase the risk of developing cardiovascular disease in adulthood (Williams et al., 2005b; Crispi et al., 2010; Giussani et al., 2012; Giussani \& Davidge, 2013).

Again, studies using the chicken embryo have shown that hypoxic incubation can recapitulate the adverse cardiovascular phenotype described in studies either using mammalian animal models of chronic hypoxia or in human clinical studies of IUGR pregnancies, supporting that chronic fetal hypoxia is an important mechanism. The relatively large size of the chicken embryo at term $(>25 \mathrm{~g}$ on day 19 , term is 21 days, Table 1) compared to the rodent fetus $(<4 \mathrm{~g}$ on day 20, term is 21 days, see Camm et al. (2010)) means that the chicken embryo model has the added advantage of facilitating study of cardiovascular function in ovo or in isolated organs, for instance using the Langendorff preparation (Itani et al., 2016b) or the myograph (Ruijtenbeek et al., 2003b; Villamor et al., 2004; Van der Sterren et al., 2009; Moonen et al., 2012; Itani et al., 2016a; Itani et al., 2016b). Interestingly, and perhaps surprisingly, the chronology of cardiac development in the chicken is much more comparable to the human than is the rodent, in which cardiac development continues into the postnatal period (Sissman, 1970; Monie, 1976; Marcela et al., 2012. See Figure 2). Combined, these advantages have convinced many investigators to use the chicken embryo as a useful animal model to isolate the effects of chronic hypoxia on cardiovascular development (Table 2). In chapter III, we reported that chicken embryos of both sea level and high altitude hens following incubation at high altitude have increased relative heart weight as well as relative left (LV) and right (RV) ventricular area and wall thickness). These embryos also had a higher aortic wall:lumen area ratio, indicating that developmental exposure to hypobaric hypoxia induced aortic hypertrophy prior to hatching. This morphometric analysis of the cardiovascular system of the high altitude chicken embryo has been 
expanded recently to include analysis on cardiovascular morphology of chicken embryos exposed to isobaric hypoxia throughout the incubation period (Itani et al., 2016b). In contrast to high altitude incubation, chronic isobaric hypoxia induced cardiac dilatation with reduced LV wall while enhancing LV lumen volume, yielding a marked reduction, rather than an increase, in the LV wall:lumen area ratio (Itani et al., 2016b). Similarly, both dilatation as well as hypertrophy of the aorta in chronically hypoxic chicken embryos have been reported by independent groups (Figure 2 and 3. Rouwet et al., 2002; Salinas et al., 2010; Itani et al., 2016b). The reasons underlying the differential effects of chronic hypobaric versus isobaric hypoxia of similar magnitude and duration on cardiovascular remodelling in the developing embryo are not clear at present.

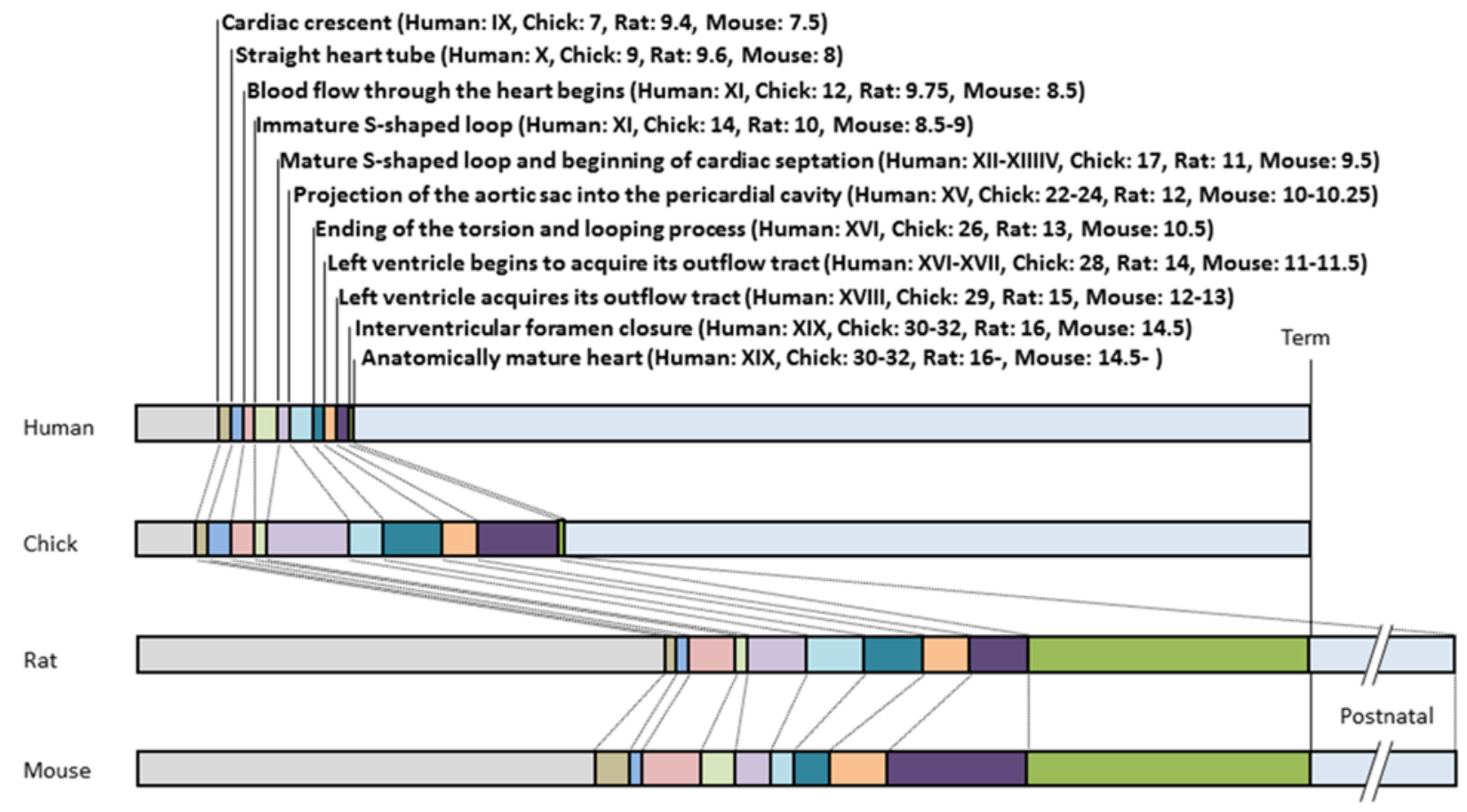

Figure 2. Comparison of the key stages of cardiac development in different animal models. Bars represent the proportion (\%) of development. Roman and Arabic numerals refer to: Human Carnegie Stage (Streeter Horizons), Chick Hamburger and Hamilton stage, Rat and Mouse embryonic days. Term: Human, 38 weeks; Chick, 21 days; Rat, 21 days; Mouse, 19 days. While the main anatomical development of the heart is complete in the rat and mouse by day 16 and 14.5 , respectively, the acquisition of mature septum, valves and tendinea cords is not yet complete until after birth (Marcela et al., 2012). Drawn from data provided in Sissman (1970) and Marcela et al. (2012). 


\begin{tabular}{|c|c|c|c|c|c|c|c|}
\hline Breed & $\begin{array}{l}\text { Study } \\
\text { design }\end{array}$ & $\begin{array}{l}\text { Temp (C), } \\
\text { Humidity } \\
\text { (\%) }\end{array}$ & $\begin{array}{l}\text { Oxygen } \\
\text { (\%) N, H }\end{array}$ & $\begin{array}{l}\text { Effects on cardiovascular } \\
\text { function }\end{array}$ & $\begin{array}{l}\text { Morphological and/or } \\
\text { histochemical effects } \\
\text { on the heart }\end{array}$ & $\begin{array}{l}\text { Morphological and/or } \\
\text { histochemical effects } \\
\text { on vessels }\end{array}$ & Reference \\
\hline $\begin{array}{l}\text { Black } \\
\text { Leghorn }\end{array}$ & $\begin{array}{l}\text { Hypobaric } \\
\text { hypoxia }\end{array}$ & 38,60 & $20,13.7$ & & $\begin{array}{l}\text { Increased HW, LV and } \\
\mathrm{RV} \text { area and wall } \\
\text { thicknesses relative to } \\
\text { body }\end{array}$ & $\begin{array}{l}\text { Increased aortic } \\
\text { wall:lumen area ratio on } \\
\text { embryonic day } 20\end{array}$ & $\begin{array}{l}\text { (Salinas et al., } \\
\text { 2010) }\end{array}$ \\
\hline $\begin{array}{l}\text { Black } \\
\text { Leghorn }\end{array}$ & $\begin{array}{l}\text { Hypobaric } \\
\text { hypoxia }\end{array}$ & & $20,13.7$ & $\begin{array}{l}\text { Significantly lower systolic, } \\
\text { diastolic and mean arterial } \\
\text { pressure at } 6 \text { months of age. } \\
\text { Increased HR and lower pulse } \\
\text { interval in the males but not } \\
\text { females }\end{array}$ & & & $\begin{array}{l}\text { (Herrera et al., } \\
\text { 2013) }\end{array}$ \\
\hline $\begin{array}{l}\text { Black } \\
\text { Leghorn }\end{array}$ & $\begin{array}{l}\text { Hypobaric } \\
\text { hypoxia }\end{array}$ & 38,60 & $20,13.7$ & $\begin{array}{l}\text { Increased tricuspid pressure } \\
\text { gradient. Reduced mitral valve } \\
\text { pressure gradient in males }\end{array}$ & $\begin{array}{l}\text { Thicker right ventricular } \\
\text { wall }\end{array}$ & $\begin{array}{l}\text { Increased pulmonary } \\
\text { artery diameter in males }\end{array}$ & $\begin{array}{l}\text { (Salinas et al., } \\
\text { 2014) }\end{array}$ \\
\hline $\begin{array}{l}\text { Bovans } \\
\text { Brown }\end{array}$ & $\begin{array}{l}\text { Isobaric } \\
\text { hypoxia }\end{array}$ & $37.9,45$ & 21,14 & $\begin{array}{l}\text { Reduced systolic pressure and } \\
\text { higher diastolic pressure, } \\
\text { impaired peripheral endothelial } \\
\text { vasodilation }\end{array}$ & LV dilatation & Aortic dilatation & $\begin{array}{l}\text { (Itani et al., } \\
\text { 2016a; Itani et } \\
\text { al., 2016b) }\end{array}$ \\
\hline $\begin{array}{l}\text { White } \\
\text { Leghorn }\end{array}$ & $\begin{array}{l}\text { Isobaric } \\
\text { hypoxia } \\
\text { from day } \\
6\end{array}$ & 38,60 & 21,15 & $\begin{array}{l}\text { Reduced sensitivity of femoral } \\
\text { artery to noradrenaline }\end{array}$ & $\begin{array}{l}\text { Increased noradrenaline } \\
\text { content }\end{array}$ & $\begin{array}{l}\text { Higher perivascular } \\
\text { sympathetic innervation } \\
\text { in femoral artery }\end{array}$ & $\begin{array}{l}\text { (Ruijtenbeek et } \\
\text { al., 2000) }\end{array}$ \\
\hline $\begin{array}{l}\text { White } \\
\text { Leghorn }\end{array}$ & $\begin{array}{l}\text { Isobaric } \\
\text { hypoxia } \\
\text { from day } \\
6\end{array}$ & $38,60-70$ & 21,15 & $\begin{array}{l}\text { Impaired NO-dependent } \\
\text { endothelial relaxation to Ach. } \\
\text { Enhanced contractile response } \\
\text { of the femoral artery to electrical } \\
\text { stimulation 14-15 weeks post } \\
\text { hatching }\end{array}$ & & $\begin{array}{l}\text { Perivascular } \\
\text { hyperinnervation no } \\
\text { longer present } 14-15 \\
\text { weeks after hatching }\end{array}$ & $\begin{array}{l}\text { (Ruijtenbeek et } \\
\text { al., 2003a) }\end{array}$ \\
\hline $\begin{array}{l}\text { White } \\
\text { Leghorn }\end{array}$ & $\begin{array}{l}\text { Isobaric } \\
\text { hypoxia } \\
\text { from day } \\
6\end{array}$ & 38,60 & 21,15 & $\begin{array}{l}\text { Impaired NO-dependent } \\
\text { vasodilatation of the femoral } \\
\text { artery on d } 19\end{array}$ & & & $\begin{array}{l}\text { (Ruijtenbeek et } \\
\text { al., 2003b) }\end{array}$ \\
\hline $\begin{array}{l}\text { White } \\
\text { Leghorn }\end{array}$ & $\begin{array}{l}\text { Isobaric } \\
\text { hypoxia } \\
\text { from day } \\
6\end{array}$ & 38,60 & 21,15 & $\begin{array}{l}\text { Impaired contractile function of } \\
\text { the pulmonary artery. Impaired } \\
\text { femoral arterial relaxation to } \\
\text { ACh, }\end{array}$ & $\begin{array}{l}\text { Increased heart mass } \\
\text { and } L V \text { and } R V \text { wall } \\
\text { thickness and areas } \\
\text { relative to body }\end{array}$ & & $\begin{array}{l}\text { (Villamor et al., } \\
\text { 2004) }\end{array}$ \\
\hline
\end{tabular}




\begin{tabular}{|c|c|c|c|c|c|c|c|}
\hline Breed & $\begin{array}{l}\text { Study } \\
\text { design }\end{array}$ & $\begin{array}{l}\text { Temp (C), } \\
\text { Humidity } \\
\text { (\%) }\end{array}$ & $\begin{array}{l}\text { Oxygen } \\
\text { (\%) N, H }\end{array}$ & $\begin{array}{l}\text { Effects on cardiovascular } \\
\text { function }\end{array}$ & $\begin{array}{l}\text { Morphological and/or } \\
\text { histochemical effects } \\
\text { on the heart }\end{array}$ & $\begin{array}{l}\text { Morphological and/or } \\
\text { histochemical effects } \\
\text { on vessels }\end{array}$ & Reference \\
\hline $\begin{array}{l}\text { White } \\
\text { Leghorn }\end{array}$ & $\begin{array}{l}\text { Isobaric } \\
\text { hypoxia } \\
\text { from day } \\
6\end{array}$ & $37.8,45$ & 21,15 & $\begin{array}{l}\text { Shortened ductus arteriosus. } \\
\text { Enhanced DA contractile } \\
\text { response to a-adrenergic } \\
\text { agonists and impaired } \\
\text { endothelial-dependent, - } \\
\text { independent and } \beta \text { - } \\
\text { adrenoreceptor agonist-induced } \\
\text { relaxation }\end{array}$ & & & $\begin{array}{l}\text { (Van der Sterren } \\
\text { et al., 2009) }\end{array}$ \\
\hline $\begin{array}{l}\text { White } \\
\text { Leghorn }\end{array}$ & $\begin{array}{l}\text { Isobaric } \\
\text { hypoxia } \\
\text { from day } \\
6\end{array}$ & $37.8,45$ & 21,15 & $\begin{array}{l}\text { Endothelium-dependent } \\
\text { relaxation of intrapulmonary } \\
\text { arteries are not affected }\end{array}$ & & & $\begin{array}{l}\text { (Zoer et al., } \\
\text { 2009) }\end{array}$ \\
\hline Broiler & $\begin{array}{l}\text { Isobaric } \\
\text { hypoxia } \\
\text { from day } \\
6\end{array}$ & $37.8,45$ & 21,15 & $\begin{array}{l}\text { Endothelium-dependent } \\
\text { relaxation of intrapulmonary } \\
\text { arteries are not affected }\end{array}$ & & & $\begin{array}{l}\text { (Zoer et al., } \\
\text { 2009) }\end{array}$ \\
\hline $\begin{array}{l}\text { White } \\
\text { Leghorn }\end{array}$ & $\begin{array}{l}\text { Isobaric } \\
\text { hypoxia }\end{array}$ & $37.8,45$ & 21,15 & $\begin{array}{l}\text { Hypoxia did not affect the Rho- } \\
\text { kinase inhibitor hydroxyfasudil- } \\
\text { induced relaxation }\end{array}$ & & & $\begin{array}{l}\text { (Zoer et al., } \\
2010)\end{array}$ \\
\hline $\begin{array}{l}\text { White } \\
\text { Leghorn }\end{array}$ & $\begin{array}{l}\text { Isobaric } \\
\text { hypoxia }\end{array}$ & $37.8,60$ & 21,15 & $\begin{array}{l}\text { Enhanced vascular response to } \\
\text { noradrenaline on } d 19 . \\
\text { Enhanced response to } \\
\text { endothelin- } 1 \text { on } d 15 \text { and } \\
\text { postnatal d } 1 \text {. Diminished } \\
\text { response to ACh on } d 15 .\end{array}$ & & & $\begin{array}{l}\text { (Moonen et al., } \\
\text { 2012) }\end{array}$ \\
\hline $\begin{array}{l}\text { White } \\
\text { Leghorn }\end{array}$ & $\begin{array}{l}\text { Isobaric } \\
\text { hypoxia }\end{array}$ & 37,60 & 21,15 & $\begin{array}{l}\text { Reduced systolic pressure, } \\
\text { enhanced peripheral arterial } \\
\text { tone }\end{array}$ & $\begin{array}{l}\text { Heart weight increased } \\
\text { relative to body, reduced } \\
\text { septum thickness }\end{array}$ & $\begin{array}{l}\text { Hypertrophic growth of } \\
\text { the aorta }\end{array}$ & $\begin{array}{l}\text { (Rouwet et al., } \\
\text { 2002) }\end{array}$ \\
\hline $\begin{array}{l}\text { White } \\
\text { Leghorn }\end{array}$ & $\begin{array}{l}\text { Isobaric } \\
\text { hypoxia }\end{array}$ & 37,60 & 21,15 & $\begin{array}{l}\text { LV systolic and diastolic } \\
\text { dysfunction at } \mathrm{d} 20 \text { and } 8 \\
\text { months post hatching }\end{array}$ & $\begin{array}{l}\text { Dilated left ventricle } \\
\text { with enhanced fibrosis, } \\
\text { cardiomyocyte } \\
\text { degeneration and } \\
\text { disorganisation }\end{array}$ & & $\begin{array}{l}\text { (Tintu et al., } \\
\text { 2009) }\end{array}$ \\
\hline $\begin{array}{l}\text { White } \\
\text { Leghorn }\end{array}$ & $\begin{array}{l}\text { Isobaric } \\
\text { hypoxia }\end{array}$ & $38,60-70$ & 21,15 & $\begin{array}{l}\text { Reduced peak systolic pressure, } \\
\text { systolic volume and cardiac } \\
\text { output }\end{array}$ & & & $\begin{array}{l}\text { (Sharma et al., } \\
\text { 2006) }\end{array}$ \\
\hline
\end{tabular}




\begin{tabular}{|c|c|c|c|c|c|c|c|}
\hline Breed & $\begin{array}{l}\text { Study } \\
\text { design }\end{array}$ & $\begin{array}{l}\text { Temp (C), } \\
\text { Humidity } \\
(\%)\end{array}$ & $\begin{array}{l}\text { Oxygen } \\
\text { (\%) N, H }\end{array}$ & $\begin{array}{l}\text { Effects on cardiovascular } \\
\text { function }\end{array}$ & $\begin{array}{l}\text { Morphological and/or } \\
\text { histochemical effects } \\
\text { on the heart }\end{array}$ & $\begin{array}{l}\text { Morphological and/or } \\
\text { histochemical effects } \\
\text { on vessels }\end{array}$ & Reference \\
\hline $\begin{array}{l}\text { Broiler } \\
\text { strain } \\
\text { Ross } \\
308\end{array}$ & $\begin{array}{l}\text { Isobaric } \\
\text { hypoxia }\end{array}$ & $37.8,45$ & $\begin{array}{l}20.95 \\
14\end{array}$ & $\begin{array}{l}\text { Decreased density and } \\
\text { enhanced sensitivity of } \beta \text {-AR at } \\
\text { d } 19 \text {, followed by decreased } \\
\text { sensitivity to } \beta \text {-AR stimulation } \\
\text { by d35 post hatching }\end{array}$ & $\begin{array}{l}\text { Increased relative heart } \\
\text { mass in the embryo and } \\
\text { in } 14 \mathrm{~d} \text { old hatchlings, } \\
\text { but the effect disappears } \\
\text { by d } 35 \text { post hatching }\end{array}$ & & $\begin{array}{l}\text { (Lindgren \& } \\
\text { Altimiras, 2009) }\end{array}$ \\
\hline $\begin{array}{l}\text { Broiler } \\
\text { strain } \\
\text { Ross } \\
308\end{array}$ & $\begin{array}{l}\text { Isobaric } \\
\text { hypoxia }\end{array}$ & $37.8,45$ & 21,14 & $\begin{array}{l}\text { Hypotension and enhanced } \beta A R \\
\text { sensitivity }\end{array}$ & & & $\begin{array}{l}\text { (Lindgren et al., } \\
\text { 2011) }\end{array}$ \\
\hline $\begin{array}{l}\text { Broiler } \\
\text { strain } \\
\text { Ross } \\
308 \\
\end{array}$ & $\begin{array}{l}\text { Isobaric } \\
\text { hypoxia }\end{array}$ & $37.8,45$ & 21,14 & $\begin{array}{l}\text { Increased } \beta 1 \mathrm{AR} \text { activity and in } \\
\text { vivo systolic dysfunction }\end{array}$ & $\begin{array}{l}\text { Increased relative heart } \\
\text { mass and increased } \\
\text { systolic lumen diameter }\end{array}$ & & $\begin{array}{l}\text { (Lindgren \& } \\
\text { Altimiras, 2013) }\end{array}$ \\
\hline $\begin{array}{l}\text { White } \\
\text { Leghorn }\end{array}$ & $\begin{array}{l}\text { Isobaric } \\
\text { hypoxia }\end{array}$ & 38,60 & 21,15 & $\begin{array}{l}\text { Blunted in vivo cardiovascular } \\
\text { response to superimposed acute } \\
\text { hypoxia and NO stimulation }\end{array}$ & & & $\begin{array}{l}\text { (Iversen et al., } \\
2014)\end{array}$ \\
\hline $\begin{array}{l}\text { White } \\
\text { Leghorn }\end{array}$ & $\begin{array}{l}\text { Isobaric } \\
\text { hypoxia }\end{array}$ & 38,60 & 21,15 & $\begin{array}{l}\text { Reduced stroke volume and } \\
\text { cardiac output, and impaired } \\
\text { left ventricular contractility and } \\
\text { relaxability }\end{array}$ & $\begin{array}{l}\text { Increased relative heart } \\
\text { and LV mass. Reduced } \\
\text { expression of the genes } \\
\text { involved in excitation- } \\
\text { contraction coupling }\end{array}$ & & $\begin{array}{l}\text { (Jonker et al., } \\
2015)\end{array}$ \\
\hline $\begin{array}{l}\text { Broiler } \\
\text { strain } \\
\text { Ross } \\
308\end{array}$ & $\begin{array}{l}\text { Isobaric } \\
\text { hypoxia }\end{array}$ & $37.8,45$ & 21,14 & & $\begin{array}{l}\text { Increased relative heart } \\
\text { mass. No effect on } \\
\text { cardiomyocyte density } \\
\text { or size, indicating a } \\
\text { reduction in the cell } \\
\text { number by d19 }\end{array}$ & & $\begin{array}{l}\text { (Osterman et al., } \\
\text { 2015) }\end{array}$ \\
\hline
\end{tabular}

Table 2. Studies showing effects of chronic developmental hypoxia on the cardiovascular system in chicken embryos and /or in adult birds. BW, body weight; HW, heart weight; LV, left ventricle; RV, right ventricle. 


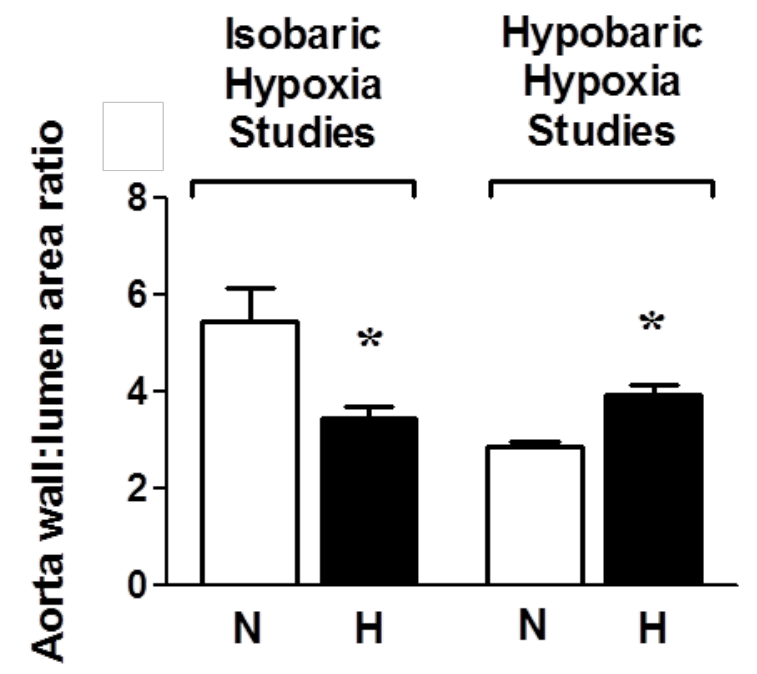

Figure 3. Aortic morphology in the chicken embryo at day 19-20 of incubation. Values are mean \pm S.E.M at day 19-20 of aorta wall:lumen area ratio of chicken embryos incubated in either normoxia $(N, n=10)$ or hypoxia $(H, n=10)$ for isobaric hypoxia studies, and normoxia at sea level $(\mathrm{N}, \mathrm{n}=8)$ or hypoxia at high altitude $(\mathrm{H}, \mathrm{n}=7)$ for hypobaric hypoxia studies. *Significantly $(\mathrm{P}<0.05)$ different from corresponding control. Data adapted from chapter III and Itani et al. (2016b).

In addition to morphological remodelling of the developing heart, a number of studies in humans and mammalian animal models have reported that chronic fetal hypoxia has pronounced adverse effects on cardiac and vascular function in the offspring. In humans, IUGR pregnancy is associated with impaired cardiac contractility and ventricular filling, together with reduced ventricular ejection force in the offspring before and after birth (Rizzo et al., 1995; Gardiner et al., 2001). Human epidemiological studies have also reported endothelial dysfunction in children and adults with low birth weight (Goodfellow et al., 1998; Martin et al., 2000; Leeson et al., 2001). In line with observations in human IUGR offspring, there are now elegant non-human primate data derived from cardiac magnetic resonance imaging that reports IUGR also being associated with cardiac remodelling in young adult baboons (Kuo et al., 2017a; Kuo et al., 2017b). Studies in rodent mammalian animal models have also reported that chronic fetal hypoxia can programme cardiac and vascular dysfunction in later life (Thompson \& Weiner, 1999; Kim et al., 2005; Williams et al., 2005a; Giussani et al., 2012; Giussani \& Davidge, 2013; Kane et al., 2013). 
Collectively, studies in the chicken embryo suggest that cardiovascular dysfunction in children and in mammalian animal models of IUGR pregnancy may again be attributable to chronic fetal hypoxia. Thus, incubation of chicken embryos under hypoxic conditions was also associated with reduced left ventricular ejection fraction and contractility, and diminished left ventricular developed pressure, all indicative of significant systolic dysfunction (Rouwet et al., 2002; Sharma et al., 2006; Tintu et al., 2009; Jonker et al., 2015; Itani et al., 2016b). Several candidate pathways may contribute to the hypoxia-induced cardiovascular dysfunction, including those involving VEGF (Tintu et al., 2009; Moonen et al., 2012; Itani et al., 2016b) and Rho-kinase (Zoer et al., 2010a). Exposure to chronic hypoxia increased both gene and protein expressions of VEGF in the embryonic heart, and systemic administration of recombinant VEGF mimicked the hypoxia-induced cardiac dilatation (Tintu et al., 2009; Itani et al., 2016b). Itani and colleagues have also reported that hypoxic incubation increases indices of oxidative stress, reduces the expression and activity of endogenous antioxidant enzymes and impairs levels of nitric oxide species in the chick embryo heart by the end of the incubation period (Itani et al., 2016 a; Itani et al., 2016b).

Incubation of chicken embryos under hypoxic conditions can also recapitulate impaired vasodilatation and/or enhanced contractile responses in pulmonary and systemic arteries in response to pharmacological or electrical stimulation (Table 2, Ruijtenbeek et al., 2003b; Villamor et al., 2004; Van der Sterren et al., 2009; Moonen et al., 2012; Itani et al., 2016a; Itani et al., 2016b). Endothelial function has been mainly studied by the use of acetylcholine (ACh). In the chicken embryo, ACh induced an endothelium-dependent and, at least partially, NO-mediated relaxation of pulmonary (Villamor et al., 2002), femoral (le Noble et al., 2000; Villamor et al., 2002), mesenteric (Moonen \& Villamor, 2011), and carotid arteries (le Noble et al., 2000), as well as in the ductus arteriosus (Agren et al., 2008). Interestingly, chronic isobaric hypoxia led to impairment of ACh-induced relaxation in the systemic (Ruijtenbeek et al., 2003a; Ruijtenbeek et al., 2003b; Villamor et al., 2004; Van der Sterren et al., 2009; Moonen et al., 2012) but not in the pulmonary arteries (Villamor et al., 2004). In contrast, chronic hypobaric hypoxia impaired endothelium-independent relaxation in the ductus arteriosus (Van der Sterren et al., 2009), did not affect it in femoral and pulmonary arteries (Ruijtenbeek et al., 2003a; Ruijtenbeek et al., 2003b; Villamor et al., 2004) and increased it in mesenteric arteries (Moonen et al., 2012). Altogether, this suggests that the effects 
of hypoxia in endothelium-dependent and -independent relaxation in chicken embryo vessels are strongly vascular bed-dependent.

Fewer studies have investigated the consequence of hypoxic incubation on the cardiovascular system of the adult bird (Table 2). Chronically instrumented adult chickens raised from eggs incubated at high altitude were significantly hypotensive with lower systolic and diastolic arterial pressures (chapter V). Adult chickens raised from eggs incubated at high altitude also showed echocardiographic indices of pulmonary hypertension and right heart dysfunction, relative to birds raised from eggs incubated at sea level (chapter VI). One important consideration for studies of cardiovascular function at high altitude in humans and experimental animals at adulthood is that the effects on cardiovascular dysfunction triggered by post-natal versus pre-natal hypoxia cannot be disentangled. However, studies from independent laboratories have reported that adult birds raised in a normoxic environment but incubated under hypoxic conditions do indeed show altered contractile and relaxant responses in the peripheral vasculature (Ruijtenbeek et al., 2003a; Ruijtenbeek et al., 2003b), systolic and diastolic dysfunction and marked increases in indices of myocardial fibrosis (Tintu et al., 2009; Lindgren \& Altimiras, 2013). These studies therefore support an effect of hypoxia during the incubation period rather than after hatching as the relevant stimulus in programing future cardiovascular risk at adulthood.

There is growing evidence for the importance of addressing sex differences in the programming of cardiovascular disease by adverse developmental conditions (Gilbert \& Nijland, 2008; Aiken \& Ozanne, 2013). The chicken embryo model offers a cost-effective experimental means to address some of these questions. Indeed, incubation of eggs at high altitude has been reported to have differential effects on cardiovascular function in male and female adult chickens (chapter V). Relative to sea level controls, hens that were incubated and raised at high altitude displayed an increased cardiac baroreflex gain, while this was significantly impaired in cockerels which were incubated and raised at high altitude (chapter V). Pre- and post-hatching development of chickens at high altitude is also associated with echocardiographic indices of pulmonary hypertension at adulthood in a highly sexdependent manner (Chapter VI). Male but not female chickens incubated and reared at high altitude had significantly greater right ventricular wall thickness during systole and diastole than their same sex sea level counterparts. Further, the tricuspid pressure gradient was greatly enhanced in highland male and female 
chickens relative to sea level controls. However, the increment in the tricuspid pressure gradient and the pulmonary artery diameter was significantly greater in highland cockerels than in highland hens. In fact, pre- and post-hatching development at high altitude did not affect the pulmonary artery diameter significantly in female chickens (chapter VI)

\section{Summary and perspectives}

In contrast to observations in humans and experiments in mammalian animal models, the direct effects of prenatal hypoxia on the individual can be isolated using the chicken embryo. Importantly, these effects on the embryonic and adult bird are independent of effects of hypoxic exposure on the maternal physiology, on the release of placental hormones and on the quality of the milk for lactation, as in mammalian species. The chicken compared to the rat or the mouse is a precocial rather than altricial species. Therefore, the temporal developmental trajectory of cardiovascular structure and function is also much more similar between chickens and humans. Data generated from chicken embryo incubations under hypoxic conditions are now beginning to reveal cellular and molecular mechanisms through which chronic hypoxia directly affects growth and the HPA axis during prenatal development and the setting of a future risk of cardiovascular, metabolic or endocrine disease. An additional advantage of the chicken embryo model is that it is high throughput and cost-effective. Therefore, interventional strategies to protect against growth restriction and the developmental programming of cardiovascular disease by chronic hypoxia can be tested in parallel, in different doses and at varying times of administration within one experimental design. Recent studies have reported that treatment of hypoxic incubations with antioxidants or agents that increase NO bioavailability, such as with melatonin or sildenafil (Itani et al., 2016a; Itani et al., 2016b), can not only protect but rescue the cardiovascular phenotype of the offspring even when therapy is started long after the induction of chronic developmental hypoxia. The latter is an important advance for human translational therapy, as in the clinical setting, IUGR resulting from chronic fetal hypoxia in adverse pregnancy needs to be diagnosed before it can be treated.

Blunting of fetal basal adrenal cortical but not medullary function may be an appropriate homeostatic response to prolonged periods of fetal hypoxia to protect sensitive tissues from sustained elevations in plasma glucocorticoid levels (Ducsay, 
1998; Myers et al., 2005). Conversely, sensitisation of the sympathoadrenal system may maintain appropriate glucogenic capacity during fetal development under conditions of chronic fetal hypoxia. The biological trade-offs of the divergent adaptations in adrenal responses may yield newborns with adrenocortical suppression and adrenergic hyper-reactivity. Both will have clear consequences for the programming of cardio-metabolic and endocrine dysfunction in later life (Reynolds et al., 2001; Peyronnet et al., 2002; Kajantie et al., 2003; Watterberg et al., 2004) and this warrants further investigation.

The chicken embryo is a valuable model for the study of the pathophysiological effects not only of chronic but also of acute hypoxia. The fetus can be exposed to acute hypoxic challenges, which if severe can lead to injury or death (Maltepe \& Saugstad, 2009; Vento et al., 2012; Giussani, 2016). Reoxygenation following exposure to hypoxia may exacerbate the hypoxic damage (Maltepe \& Saugstad, 2009; Vento et al., 2012). Either in the mammalian fetus or the chicken embryo the cardiovascular responses to acute hypoxia includes a redistribution of the cardiac output away from the periphery towards high priority organs such as the heart, brain, and adrenal glands (Giussani et al., 1993; Mulder et al., 2000; Mulder et al., 2002; Giussani, 2016). An important component of hypoxia-induced flow redistribution is mediated by increased release of catecholamines (Mulder et al., 2000; Mulder et al., 2001; Mulder et al., 2002; Fletcher et al., 2006), whose effects are modulated by the different responsiveness of the vascular beds. However, hypoxia-mediated redistribution of blood flow is also accomplished by a local vascular effect, which results in contraction or relaxation depending on the blood vessel. Hypoxia-induced contraction of isolated pulmonary artery (Zoer et al., 2009; van der Sterren et al., 2011; Moreno et al., 2014) and mesenteric artery (Brinks et al., 2016), and hypoxic relaxation of isolated femoral artery (Zoer et al., 2010b; van der Sterren et al., 2011) and ductus arteriosus (Agren et al., 2007; Greyner \& Dzialowski, 2008; Cogolludo et al., 2009; van der Sterren et al., 2011; Van der Sterren et al., 2014) have been described in chicken embryos. In addition, the effects of chronic in ovo hypoxia on the ex vivo responsiveness of these vessels to acute hypoxia as well as to vasoactive mediators have been extensively studied (Van der Sterren et al., 2009; Zoer et al., 2009; Zoer et al., 2010b; Lindgren et al., 2011; Moonen et al., 2012). However, the effects of acute in ovo hypoxia-reoxygenation on vascular reactivity have not been yet characterized and warrant further investigation. 


\section{References}

Agren P, Cogolludo AL, Kessels CG, Perez-Vizcaino F, De Mey JG, Blanco CE \& Villamor E. (2007). Ontogeny of chicken ductus arteriosus response to oxygen and vasoconstrictors. Am J Physiol Regul Integr Comp Physiol 292, R485-496.

Agren P, van der Sterren S, Cogolludo AL, Frazziano G, de Mey JG, Blanco CE \& Villamor E. (2008). Developmental changes in endothelium-dependent relaxation of the chicken ductus arteriosus. J Physiol Pharmacol 59, 55-76.

Aiken CE \& Ozanne SE. (2013). Sex differences in developmental programming models. Reproduction 145, R1-13.

Akira M \& Yoshiyuki S. (2006). Placental circulation, fetal growth, and stiffness of the abdominal aorta in newborn infants. J Pediatr 148, 49-53.

Allison BJ, Brain KL, Niu Y, Kane AD, Herrera EA, Thakor AS, Botting KJ, Cross CM, Itani N, Skeffington KL, Beck C \& Giussani DA. (2016). Fetal in vivo continuous cardiovascular function during chronic hypoxia. J Physiol 594, 1247-1264.

Arnett DK, Evans GW \& Riley WA. (1994). Arterial stiffness: a new cardiovascular risk factor? Am J Epidemiol 140, 669-682.

Brinks L, Moonen RM, Moral-Sanz J, Barreira B, Kessels L, Perez-Vizcaino F, Cogolludo A \& Villamor E. (2016). Hypoxia-induced contraction of chicken embryo mesenteric arteries: mechanisms and developmental changes. Am J Physiol Regul Integr Comp Physiol, ajpregu. 00461.02015.

Camm EJ, Hansell JA, Kane AD, Herrera EA, Lewis C, Wong S, Morrell NW \& Giussani DA. (2010). Partial contributions of developmental hypoxia and undernutrition to prenatal alterations in somatic growth and cardiovascular structure and function. Am J Obstet Gynecol 203, 495 e424-434.

Challis JR, Fraher L, Oosterhuis J, White SE \& Bocking AD. (1989). Fetal and maternal endocrine responses to prolonged reductions in uterine blood flow in pregnant sheep. Am J Obstet Gynecol 160, 926-932.

Cogolludo AL, Moral-Sanz J, van der Sterren S, Frazziano G, van Cleef AN, Menendez C, Zoer B, Moreno E, Roman A, Perez-Vizcaino F \& Villamor E. (2009). Maturation of O2 sensing and signaling in the chicken ductus arteriosus. Am J Physiol Lung Cell Mol Physiol 297, L619-630.

Cosmi E, Visentin S, Fanelli T, Mautone AJ \& Zanardo V. (2009). Aortic intima media thickness in fetuses and children with intrauterine growth restriction. Obstet Gynecol 114, 1109-1114.

Crispi F, Bijnens B, Figueras F, Bartrons J, Eixarch E, Le Noble F, Ahmed A \& Gratacos E. (2010). Fetal growth restriction results in remodeled and less efficient hearts in children. Circulation 121, 2427-2436. 
Ducsay CA. (1998). Fetal and maternal adaptations to chronic hypoxia: prevention of premature labor in response to chronic stress. Comp Biochem Physiol A Mol Integr Physiol 119, 675-681.

Dzialowski EM, von Plettenberg D, Elmonoufy NA \& Burggren WW. (2002). Chronic hypoxia alters the physiological and morphological trajectories of developing chicken embryos. Comp Biochem Physiol A Mol Integr Physiol 131, 713-724.

Fletcher AJ, Gardner DS, Edwards CMB, Fowden AL \& Giussani DA. (2006). Development of the ovine fetal cardiovascular defense to hypoxemia towards full term. Am J Physiol Heart Circ Physiol 291, H3023-H3034.

Fowden AL, Giussani DA \& Forhead AJ. (2006). Intrauterine programming of physiological systems: causes and consequences. Physiol (Bethesda) 21, 2937.

Gardiner H, Brodszki J \& Marsal K. (2001). Ventriculovascular physiology of the growth-restricted fetus. Ultrasound Obstet Gynecol 18, 47-53.

Gardner DS, Fletcher AJ, Bloomfield MR, Fowden AL \& Giussani DA. (2002). Effects of prevailing hypoxaemia, acidaemia or hypoglycaemia upon the cardiovascular, endocrine and metabolic responses to acute hypoxaemia in the ovine fetus. J Physiol 540, 351-366.

Gilbert JS \& Nijland MJ. (2008). Sex differences in the developmental origins of hypertension and cardiorenal disease. Am J Physiol Regul Integr Comp Physiol 295, R1941-1952.

Giussani D, Spencer J, Moore P, Bennet L \& Hanson M. (1993). Afferent and efferent components of the cardiovascular reflex responses to acute hypoxia in term fetal sheep. J Physiol 461, 431-449.

Giussani DA. (2007). Hypoxia, fetal growth and early origins of disease: the Andean curse on the Conquistadors. J Physiol 582, 472.

Giussani DA. (2016). The fetal brain sparing response to hypoxia: physiological mechanisms. J Physiol 594, 1215-1230.

Giussani DA, Camm EJ, Niu Y, Richter HG, Blanco CE, Gottschalk R, Blake EZ, Horder KA, Thakor AS, Hansell JA, Kane AD, Wooding FB, Cross CM \& Herrera EA. (2012). Developmental programming of cardiovascular dysfunction by prenatal hypoxia and oxidative stress. PLoS One 7, e31017.

Giussani DA \& Davidge ST. (2013). Developmental programming of cardiovascular disease by prenatal hypoxia. J Dev Orig Health Dis 4, 328-337.

Giussani DA, Phillips PS, Anstee S \& Barker DJ. (2001). Effects of altitude versus economic status on birth weight and body shape at birth. Pediatr Res 49, 490-494.

Giussani DA, Salinas CE, Villena M \& Blanco CE. (2007). The role of oxygen in prenatal growth: studies in the chick embryo. J Physiol 585, 911-917. 
Gluckman PD, Hanson MA, Cooper C \& Thornburg KL. (2008). Effect of in utero and early-life conditions on adult health and disease. N Engl J Med 359, 6173.

Goodfellow J, Bellamy MF, Gorman ST, Brownlee M, Ramsey MW, Lewis MJ, Davies DP \& Henderson AH. (1998). Endothelial function is impaired in fit young adults of low birth weight. Cardiovasc Res 40, 600-606.

Greyner H \& Dzialowski EM. (2008). Mechanisms mediating the oxygen-induced vasoreactivity of the ductus arteriosus in the chicken embryo. Am J Physiol Regul Integr Comp Physiol 295, R1647-1659.

Harvey LM, Gilbert RD, Longo LD \& Ducsay CA. (1993). Changes in ovine fetal adrenocortical responsiveness after long-term hypoxemia. Am J Physiol 264, E741-747.

Hooper SB, Coulter CL, Deayton JM, Harding R \& Thorburn GD. (1990). Fetal endocrine responses to prolonged hypoxemia in sheep. Am J Physiol 259, R703-708.

Itani N, Skeffington KL, Beck C \& Giussani DA. (2016a). Sildenafil therapy for fetal cardiovascular dysfunction during hypoxic development: studies in the chick embryo. J Physiol, In press (DOI: 10.1113/JP273393).

Itani N, Skeffington KL, Beck C, Niu Y \& Giussani DA. (2016b). Melatonin rescues cardiovascular dysfunction during hypoxic development in the chick embryo. J Pineal Res 60, 16-26.

Jenkins SA \& Porter TE. (2004). Ontogeny of the hypothalamo-pituitaryadrenocortical axis in the chicken embryo: a review. Domest Anim Endocrinol 26, 267-275.

Jonker SS, Giraud GD, Espinoza HM, Davis EN \& Crossley DA, 2nd. (2015). Effects of chronic hypoxia on cardiac function measured by pressure-volume catheter in fetal chickens. Am J Physiol Regul Integr Comp Physiol 308, R680-689.

Kajantie E, Dunkel L, Turpeinen U, Stenman UH, Wood PJ, Nuutila M \& Andersson S. (2003). Placental 11 beta-hydroxysteroid dehydrogenase-2 and fetal cortisol/cortisone shuttle in small preterm infants. J Clin Endocrinol Metab 88, 493-500.

Kalliecharan R \& Hall BK. (1974). A developmental study of the levels of progesterone, corticosterone, cortisol, and cortisone circulating in plasma of chick embryos. Gen Comp Endocrinol 24, 364-372.

Kane AD, Herrera EA, Camm EJ \& Giussani DA. (2013). Vitamin C prevents intrauterine programming of in vivo cardiovascular dysfunction in the rat. Circ J 77, 2604-2611.

Kim YH, Veille JC, Cho MK, Kang MS, Kim CH, Song TB \& Figueroa JP. (2005). Chronic hypoxia alters vasoconstrictive responses of femoral artery in the fetal sheep. J Korean Med Sci 20, 13-19. 
Lang U, Baker RS, Khoury J \& Clark KE. (2000). Effects of chronic reduction in uterine blood flow on fetal and placental growth in the sheep. Am J Physiol Regul Integr Comp Physiol 279, R53-59.

le Noble FA, Ruijtenbeek K, Gommers S, de Mey JG \& Blanco CE. (2000). Contractile and relaxing reactivity in carotid and femoral arteries of chicken embryos. Am J Physiol Heart Circ Physiol 278, H1261-1268.

Leeson CP, Kattenhorn M, Morley R, Lucas A \& Deanfield JE. (2001). Impact of low birth weight and cardiovascular risk factors on endothelial function in early adult life. Circulation 103, 1264-1268.

Lindgren I \& Altimiras J. (2009). Chronic prenatal hypoxia sensitizes betaadrenoceptors in the embryonic heart but causes postnatal desensitization. Am J Physiol Regul Integr Comp Physiol 297, R258-R264.

Lindgren I \& Altimiras J. (2011). Sensitivity of organ growth to chronically low oxygen levels during incubation in Red Junglefowl and domesticated chicken breeds. Poult Sci 90, 126-135.

Lindgren I \& Altimiras J. (2013). Prenatal hypoxia programs changes in betaadrenergic signaling and postnatal cardiac contractile dysfunction. Am J Physiol Regul Integr Comp Physiol 305, R1093-1101.

Lindgren I, Crossley D, 2nd, Villamor E \& Altimiras J. (2011). Hypotension in the chronically hypoxic chicken embryo is related to the beta-adrenergic response of chorioallantoic and femoral arteries and not to bradycardia. Am J Physiol Regul Integr Comp Physiol 301, R1161-1168.

Llanos AJ, Riquelme RA, Sanhueza EM, Hanson MA, Blanco CE, Parer JT, Herrera EA, Pulgar VM, Reyes RV, Cabello G \& Giussani DA. (2003). The fetal llama versus the fetal sheep: different strategies to withstand hypoxia. High Alt Med Biol 4, 193-202.

Maltepe E \& Saugstad OD. (2009). Oxygen in health and disease: regulation of oxygen homeostasis-clinical implications. Pediatric Res 65, 261-268.

Marcela SG, Cristina RM, Angel PG, Manuel AM, Sofia DC, Patricia de LR, Bladimir RR \& Concepcion SG. (2012). Chronological and morphological study of heart development in the rat. Anat Rec (Hoboken) 295, 1267-1290.

Martin H, Hu J, Gennser G \& Norman M. (2000). Impaired endothelial function and increased carotid stiffness in 9-year-old children with low birthweight. Circulation 102, 2739-2744.

Miller SL, Green LR, Peebles DM, Hanson MA \& Blanco CE. (2002). Effects of chronic hypoxia and protein malnutrition on growth in the developing chick. Am J Obstet Gynecol 186, 261-267.

Monau TR, Vargas VE, King N, Yellon SM, Myers DA \& Ducsay CA. (2009). Longterm hypoxia increases endothelial nitric oxide synthase expression in the ovine fetal adrenal. Reprod Sci 16, 865-874. 
Monie IW. (1976). Comparative development of the nervous, respiratory, and cardiovascular systems. Environ Health Perspect 18, 55-60.

Moonen RM, Kessels CG, Zimmermann LJ \& Villamor E. (2012). Mesenteric artery reactivity and small intestine morphology in a chicken model of hypoxiainduced fetal growth restriction. J Physiol Pharmacol 63, 601-612.

Moonen RM \& Villamor E. (2011). Developmental changes in mesenteric artery reactivity in embryonic and newly hatched chicks. J Comp Physiol B 181, 1063-1073.

Moore LG, Shriver M, Bemis L, Hickler B, Wilson M, Brutsaert T, Parra E \& Vargas E. (2004). Maternal adaptation to high-altitude pregnancy: an experiment of nature--a review. Placenta 25 Suppl A, S60-71.

Moreno L, Moral-Sanz J, Morales-Cano D, Barreira B, Moreno E, Ferrarini A, Pandolfi R, Ruperez FJ, Cortijo J, Sanchez-Luna M, Villamor E, PerezVizcaino F \& Cogolludo A. (2014). Ceramide mediates acute oxygen sensing in vascular tissues. Antioxid Redox Signal 20, 1-14.

Mulder A, Golde J, Goor Av, Giussani D \& Blanco C. (2000). Developmental changes in plasma catecholamine concentrations during normoxia and acute hypoxia in the chick embryo. J Physiol 527, 593-599.

Mulder A, Miedema A, De Mey J, Giussani DA \& Blanco CE. (2002). Sympathetic control of the cardiovascular response to acute hypoxemia in the chick embryo. Am J Physiol Regul Integr Comp Physiol 282, R1156-R1163.

Mulder AL, van Golde JC, Prinzen FW \& Blanco CE. (1998). Cardiac output distribution in response to hypoxia in the chick embryo in the second half of the incubation time. J Physiol 508 ( Pt 1), 281-287.

Mulder AL, van Goor CA, Giussani DA \& Blanco CE. (2001). Alpha-adrenergic contribution to the cardiovascular response to acute hypoxemia in the chick embryo. Am J Physiol Regul Integr Comp Physiol 281, R2004-2010.

Murotsuki J, Gagnon R, Matthews SG \& Challis JR. (1996). Effects of long-term hypoxemia on pituitary-adrenal function in fetal sheep. Am J Physiol 271, E678-685.

Myers DA \& Ducsay CA. (2012). Adrenocortical and adipose responses to highaltitude-induced, long-term hypoxia in the ovine fetus. J Pregnancy 2012, 681306.

Myers DA, Hyatt K, Mlynarczyk M, Bird IM \& Ducsay CA. (2005). Long-term hypoxia represses the expression of key genes regulating cortisol biosynthesis in the near-term ovine fetus. Am J Physiol Regul Integr Comp Physiol 289, R1707-1714.

Peyronnet J, Dalmaz Y, Ehrstrom M, Mamet J, Roux JC, Pequignot JM, Thoren HP \& Lagercrantz H. (2002). Long-lasting adverse effects of prenatal hypoxia on developing autonomic nervous system and cardiovascular parameters in rats. Pflugers Arch 443, 858-865. 
Reynolds RM, Walker BR, Syddall HE, Andrew R, Wood PJ, Whorwood CB \& Phillips DI. (2001). Altered control of cortisol secretion in adult men with low birth weight and cardiovascular risk factors. J Clin Endocrinol Metab 86, 245-250.

Rizzo G, Capponi A, Rinaldo D, Arduini D \& Romanini C. (1995). Ventricular ejection force in growth-retarded fetuses. Ultrasound Obstet Gynecol 5, 247255.

Roigas J, Roigas C, Heydeck D \& Papies B. (1996). Prenatal hypoxia alters the postnatal development of beta-adrenoceptors in the rat myocardium. Biol Neonate 69, 383-388.

Rouwet EV, Tintu AN, Schellings MW, van Bilsen M, Lutgens E, Hofstra L, Slaaf DW, Ramsay G \& Le Noble FA. (2002). Hypoxia induces aortic hypertrophic growth, left ventricular dysfunction, and sympathetic hyperinnervation of peripheral arteries in the chick embryo. Circulation 105, 2791-2796.

Ruijtenbeek K, Kessels CG, Janssen BJ, Bitsch NJ, Fazzi GE, Janssen GM, De Mey $\mathrm{J} \&$ Blanco CE. (2003a). Chronic moderate hypoxia during in ovo development alters arterial reactivity in chickens. Pflugers Arch 447, 158167.

Ruijtenbeek K, Kessels LC, De Mey JG \& Blanco CE. (2003b). Chronic moderate hypoxia and protein malnutrition both induce growth retardation, but have distinct effects on arterial endothelium-dependent reactivity in the chicken embryo. Pediatr Res 53, 573-579.

Ruijtenbeek K, le Noble FA, Janssen GM, Kessels CG, Fazzi GE, Blanco CE \& De Mey JG. (2000). Chronic hypoxia stimulates periarterial sympathetic nerve development in chicken embryo. Circulation 102, 2892-2897.

Sahan U, Ipek A, Yilmaz-Dikmen B, Aydin C \& Kederli E. (2011). Effect of oxygen supplementation in the hatcher at high altitude on the incubation results of broiler eggs laid at low altitude. Br Poult Sci 52, 388-394.

Salinas CE, Blanco CE, Villena M, Camm EJ, Tuckett JD, Weerakkody RA, Kane AD, Shelley AM, Wooding FB, Quy M \& Giussani DA. (2010). Cardiac and vascular disease prior to hatching in chick embryos incubated at high altitude. J Dev Orig Health Dis 1, 60-66.

Sharma SK, Lucitti JL, Nordman C, Tinney JP, Tobita K \& Keller BB. (2006). Impact of hypoxia on early chick embryo growth and cardiovascular function. Pediatr Res 59, 116-120.

Simonetta G, Rourke AK, Owens JA, Robinson JS \& McMillen IC. (1997). Impact of placental restriction on the development of the sympathoadrenal system. Pediatr Res 42, 805-811.

Sissman NJ. (1970). Developmental landmarks in cardiac morphogenesis: comparative chronology. Am J Cardiol 25, 141-148. 
Skilton MR, Evans N, Griffiths KA, Harmer JA \& Celermajer DS. (2005). Aortic wall thickness in newborns with intrauterine growth restriction. Lancet 365, 1484-1486.

Soria R, Julian CG, Vargas E, Moore LG \& Giussani DA. (2013). Graduated effects of high-altitude hypoxia and highland ancestry on birth size. Pediatr Res 74, 633-638.

Stratford LL \& Hooper SB. (1997). Effect of hypoxemia on tissue glycogen content and glycolytic enzyme activities in fetal sheep. Am J Physiol 272, R103-110.

Thompson JA, Richardson BS, Gagnon R \& Regnault TR. (2011). Chronic intrauterine hypoxia interferes with aortic development in the late gestation ovine fetus. J Physiol 589, 3319-3332.

Thompson LP \& Weiner CP. (1999). Effects of acute and chronic hypoxia on nitric oxide-mediated relaxation of fetal guinea pig arteries. Am J Obstet Gynecol $181,105-111$.

Tintu A, Rouwet E, Verlohren S, Brinkmann J, Ahmad S, Crispi F, van Bilsen M, Carmeliet P, Staff AC, Tjwa M, Cetin I, Gratacos E, Hernandez-Andrade E, Hofstra L, Jacobs M, Lamers WH, Morano I, Safak E, Ahmed A \& le Noble F. (2009). Hypoxia induces dilated cardiomyopathy in the chick embryo: mechanism, intervention, and long-term consequences. PLoS One 4, e5155.

Van der Sterren S, Agren P, Zoer B, Kessels L, Blanco CE \& Villamor E. (2009). Morphological and functional alterations of the ductus arteriosus in a chicken model of hypoxia-induced fetal growth retardation. Pediatr Res 65, 279-284.

Van der Sterren S, Kessels L, Perez-Vizcaino F, Cogolludo A \& Villamor E. (2014). Prenatal exposure to hyperoxia modifies the thromboxane prostanoid receptor-mediated response to $\mathrm{H} 2 \mathrm{O} 2$ in the ductus arteriosus of the chicken embryo. J Physiol Pharmacol 65, 283-293.

van der Sterren S, Kleikers P, Zimmermann LJ \& Villamor E. (2011). Vasoactivity of the gasotransmitters hydrogen sulfide and carbon monoxide in the chicken ductus arteriosus. Am J Physiol Regul Integr Comp Physiol 301, R11861198.

Veille JC, Hanson R, Sivakoff M, Hoen H \& Ben-Ami M. (1993). Fetal cardiac size in normal, intrauterine growth retarded, and diabetic pregnancies. Am J Perinatol 10, 275-279.

Vento M, Escobar J, Cernada M, Escrig R \& Aguar M. (2012). The use and misuse of oxygen during the neonatal period. Clin Perinatol 39, 165-176.

Villamor E, Kessels CG, Ruijtenbeek K, van Suylen RJ, Belik J, de Mey JG \& Blanco CE. (2004). Chronic in ovo hypoxia decreases pulmonary arterial contractile reactivity and induces biventricular cardiac enlargement in the chicken embryo. Am J Physiol Regul Integr Comp Physiol 287, R642-651. 
Villamor E, Ruijtenbeek K, Pulgar V, De Mey JG \& Blanco CE. (2002). Vascular reactivity in intrapulmonary arteries of chicken embryos during transition to ex ovo life. Am J Physiol Regul Integr Comp Physiol 282, R917-927.

Watterberg KL, Gerdes JS, Cole CH, Aucott SW, Thilo EH, Mammel MC, Couser RJ, Garland JS, Rozycki HJ, Leach CL, Backstrom C \& Shaffer ML. (2004). Prophylaxis of early adrenal insufficiency to prevent bronchopulmonary dysplasia: a multicenter trial. Pediatrics 114, 1649-1657.

Wei ZH, Zhang H, Jia CL, Ling Y, Gou X, Deng XM \& Wu CX. (2007). Blood gas, hemoglobin, and growth of Tibetan chicken embryos incubated at high altitude. Poult Sci 86, 904-908.

Williams SJ, Campbell ME, McMillen IC \& Davidge ST. (2005a). Differential effects of maternal hypoxia or nutrient restriction on carotid and femoral vascular function in neonatal rats. Am J Physiol Regul Integr Comp Physiol 288, R360-367.

Williams SJ, Hemmings DG, Mitchell JM, McMillen IC \& Davidge ST. (2005b). Effects of maternal hypoxia or nutrient restriction during pregnancy on endothelial function in adult male rat offspring. J Physiol 565, 125-135.

Wise PM \& Frye BE. (1973). Functional development of the hypothalamohypophyseal-adrenal cortex axis in the chick embryo, Gallus domesticus. J Exp Zool 185, 277-292.

Woods JE, De Vries GW \& Thommes RC. (1971). Ontogenesis of the pituitaryadrenal axis in the chick embryo. Gen Comp Endocrinol 17, 407-415.

Zoer B, Blanco CE \& Villamor E. (2010a). Role of Rho-kinase in mediating contraction of chicken embryo femoral arteries. J Comp Physiol B 180, 427435.

Zoer B, Cogolludo AL, Perez-Vizcaino F, De Mey JG, Blanco CE \& Villamor E. (2010b). Hypoxia sensing in the fetal chicken femoral artery is mediated by the mitochondrial electron transport chain. Am J Physiol Regul Integr Comp Physiol 298, R1026-1034.

Zoer B, Kessels L, Vereijken A, De Mey JG, Bruggeman V, Decuypere E, Blanco CE \& Villamor E. (2009). Effects of prenatal hypoxia on pulmonary vascular reactivity in chickens prone to pulmonary hypertension. J Physiol Pharmacol 60, 119-130. 


$$
-
$$





\section{Summary}

The present thesis is a collection of studies designed to isolate the effects of chronic fetal hypoxia on fetal growth, fetal cardiovascular and endocrine development and programming of future cardiovascular dysfunction in the adult offspring, using the chicken as an animal model. The combination of high altitude exposure with the use of the chicken embryo model is ideal as it permits investigation of the direct effects of high altitude hypoxia on growth and on cardiovascular development completely independent of alterations in placental function, independent of changes in the maternal physiology and independent of any effects of socioeconomic factors.

In chapter II (The role of oxygen in prenatal growth: studies in the chick embryo. $J$ Physiol. 2007; 585:911-7), chapter III (Cardiac and vascular disease prior to hatching in chick embryos incubated at high altitude. J Dev Orig Health Dis. 2010; 1:60-6), and chapter IV (Adrenocortical suppression in highland chick embryos is restored during incubation at sea level. High Alt Med Biol. 2011; 12:79-87), we investigated the effects of high altitude hypoxia on chicken embryo growth and in ovo cardiovascular and endocrine development. For this purpose, we adopted an experimental design based on a three-prong approach using: (1) incubation at high altitude of fertilized eggs laid by sea-level hens; (2) incubation at sea level of fertilized eggs laid by high-altitude hens; and (3) incubation at high altitude of sea-level eggs with oxygen supplementation to equate sea level oxygen partial pressure. The data show that: (1) high-altitude hypoxia promotes embryonic cardiac and vascular disease already evident prior to hatching and that this is associated with growth restriction; (2) the effects can be prevented by increased oxygenation; and (3) the effects are different in embryos from sea-level or high-altitude hens. We conclude that fetal oxygenation, independent of maternal nutrition during development, has a predominant role in the control of fetal growth and cardiovascular development. Further, prolonged high altitude residence confers protection against the deleterious effects of hypoxia.

In chapter $\mathbf{V}$ (High altitude hypoxia and blood pressure dysregulation in adult chickens. J Dev Orig Health Dis. 2013; 4:69-76) and chapter VI (High-altitude hypoxia and echocardiographic indices of pulmonary hypertension in male and female chickens at adulthood. Circ J. 2014;78:1459-64), we isolated the long-term consequences of chronic hypoxic incubation of chick embryos on the systemic and pulmonary circulations of the adult bird. This was achieved using noninvasive 
echocardiography as well as testing basal and stimulated cardiovascular function in the chronically instrumented adult bird. Additional specific points of interest were to determine whether there were any sex differences and whether any adverse effects of chronic hypoxia during the embryonic period could be ameliorated by generational exposure to hypobaric hypoxia in highland adapted chickens. We show that development at high altitude hypoxia lowers basal arterial blood pressure, alters baroreflex sensitivity, and induces pulmonary hypertension in a sex-dependent manner at adulthood.

Finally, in chapter VII (The highs and lows of programmed cardiovascular disease by developmental hypoxia: Studies in the chicken embryo. J Physiol 2017), we discuss and put into perspective the findings of this thesis. We summarise studies that have exploited the chicken embryo model to isolate the direct effects of chronic hypoxia on prenatal growth, cardiovascular and endocrine development and in triggering an increased risk of cardiovascular dysfunction and pathology at adulthood. 


$$
-
$$





\section{Samenvatting}

Het huidige proefschrift is een verzameling van studies die zijn ontworpen om de effecten te isoleren van chronische foetale hypoxie op de foetale groei, foetale cardiovasculaire en endocriene ontwikkeling en programmering van toekomstige cardiovasculaire dysfunctie bij volwassen nakomelingen, waarbij de kip als een diermodel wordt gebruikt. De combinatie van blootstelling op grote hoogte met het gebruik van het kippenembryo-model is ideaal omdat het onderzoek van de directe effecten van hypoxie op grote hoogte op groei en op cardiovasculaire ontwikkeling volledig onafhankelijk van veranderingen in placenta-functie mogelijk maakt, onafhankelijk van veranderingen in de maternale fysiologie en onafhankelijk van de effecten van sociaaleconomische factoren.

In hoofdstuk II ( $J$ Physiol. 2007; 585:911-7), hoofdstuk III ( $J$ Dev Orig Health Dis. 2010; 1:60-6) en hoofdstuk IV (High Alt Med Biol. 2011; 12:79-87), hebben we de effecten onderzocht van hypoxie op grote hoogte op de groei van kippenembryo en in ovo cardiovasculaire en endocriene ontwikkeling. Voor dit doel hebben we een experimenteel ontwerp aangenomen op basis van een drieledige aanpak met behulp van: (1) incubatie op grote hoogte van bevruchte eieren gelegd door kippen op zeeniveau; (2) incubatie op zeeniveau van bevruchte eieren gelegd door kippen op grote hoogte; en (3) incubatie op grote hoogte van eieren gelegd op zeeniveau met zuurstofsuppletie om de partiële zuurstofdruk op zeeniveau gelijk te stellen. De gegevens tonen aan dat: (1) hypoxie op grote hoogte embryonale hart- en vaatziekte reeds voorafgaand aan het uitkomen van de eieren bevordert en dat dit geassocieerd is met groeirestrictie; (2) de effecten kunnen worden voorkomen door verhoogde oxygenatie; en (3) de effecten verschillen in embryo's van zeeniveau- of hooggelegen kippen. We concluderen dat foetale oxygenatie, onafhankelijk van maternale voeding tijdens de ontwikkeling, een overheersende rol speelt in de beperking van foetale groei en cardiovasculaire ontwikkeling. Verder verleent langdurig verblijf op grote hoogte bescherming tegen de schadelijke effecten van hypoxie.

In hoofdstuk V ( $J$ Dev Orig Health Dis. 2013; 4:69-76) en hoofdstuk VI (Circ J. 2014;78:1459-64), isoleerden we de langetermijngevolgen van chronische hypoxische incubatie van kippenembryo's op de systemische en pulmonale circulaties van de volwassen vogel. Dit werd bereikt met behulp van niet-invasieve 
echocardiografie en het testen van de basale en gestimuleerde cardiovasculaire functie bij de chronisch geïnstrumenteerde volwassen vogel. Aanvullende specifieke aandachtspunten waren om te bepalen of er sekse-verschillen waren en of eventuele nadelige effecten van chronische hypoxie gedurende de embryonale periode konden worden verlicht door generationele blootstelling aan hypobare hypoxie bij op hoogte aangepaste kippen. We laten zien dat ontwikkeling bij hypoxie op grote hoogte de basale arteriële bloeddruk verlaagt, de baroreflexgevoeligheid wijzigt en pulmonale hypertensie op een geslacht-afhankelijke manier induceert op volwassen leeftijd.

Tot slot bespreken we in hoofdstuk VII ( $J$ Physiol 2017) de bevindingen van dit proefschrift. We vatten studies samen die het kippenembryo-model hebben geëxploiteerd om de directe effecten van chronische hypoxie op de prenatale groei, cardiovasculaire en endocriene ontwikkeling te isoleren en om op volwassen leeftijd een verhoogd risico op cardiovasculaire disfunctie en pathologie te veroorzaken. 


$$
-
$$





\section{Relevance}

Cardiovascular disease kills 1 in 3 people. Every 3 minutes someone in the UK has a heart attack and $30 \%$ of these are fatal . Globally, around 17 million people die from cardiovascular disease each year. In 2010, the total costs of cardiovascular disease in the USA was $\$ 444$ billion and this number is predicted to increase to 800 billion dollars by 2030. In the UK, according to the British Heart Foundation, the annual costs to the nation of premature death, lost productivity, hospital treatment and prescriptions relating to cardiovascular disease is of the order of $£ 19$ billion. Therefore, there is no question that cardiovascular dysfunction is a vast problem imposing a significant burden on every country's health and wealth (1).

It is widely accepted that our genes interact with traditional lifestyle factors, such as smoking, obesity and/or a sedentary lifestyle to promote an increased risk of heart disease (2). It has also become established that the gene-environment interaction early in life may be just as, if not more, important in 'programming' heart health and heart disease (3). This is unsurprising because our physiology is much more plastic and malleable during early life and the younger we are, the greater the impact the environment has upon us (3). These concepts have brought attention to adverse pregnancy and whether it can increase the risk of cardiovascular disease in the offspring. Accordingly, we now know that fetal development during suboptimal conditions can indeed trigger a fetal origin of cardiovascular dysfunction and increase the risk of chronic heart disease in the offspring in later life (3-5).

One of the most common adverse intrauterine conditions in complicated pregnancy is chronic fetal hypoxia (4-6). However, the contribution of chronic fetal hypoxia in promoting intrauterine growth restriction (IUGR) and programmed cardiovascular risk has been difficult to isolate for a number of reasons. For instance, it is established that high altitude pregnancy leads to IUGR (5). However, most high altitude populations are impoverished with significant maternal malnutrition (5). Therefore, the contribution of chronic fetal hypoxia versus chronic fetal undernutrition in slowing fetal growth and in setting future cardiovascular risk under these conditions is uncertain. The same applies to sea level pregnancy complicated by preeclampsia, placental insufficiency, gestational diabetes and even maternal obesity. All these conditions are associated with an increase in placental 
vascular resistance (see 6), which will decrease oxygen as well as nutrient delivery to the growing fetus. This makes it impossible to disentangle the effects of chronic hypoxia versus chronic fetal undernutrition in promoting IUGR and programming of cardiovascular disease in human complicated pregnancy at sea level. Similarly, several experimantal studies including our own in mammalian animal models have shown that maternal chronic hypoxia during pregnancy can lead to IUGR and programme increased cardiovascular risk in the offspring $(5,7)$. However, because experimental induction of chronic hypoxia in rodents can reduce maternal food intake and/or alter the quality of the maternal milk $(5,7)$, the contribution of chronic fetal hypoxia versus chronic fetal and/or neonatal under-nutrition under these conditions, again, remains uncertain.

\section{Innovation}

By combining the chick embryo model with incubation at high altitude, this $\mathrm{PhD}$ thesis has been able to isolate the direct effects of chronic hypoxia due to high altitude on fetal growth, cardiovascular development, effects on the fetal stress axes and on the developmental programming of systemic and pulmonary vascular disease in the offspring. This is because in contrast to mammals, with the exception of monotremes, in the chicken the effects of changes in oxygenation on the embryo can be isolated and determined directly, independent of effects of chronic hypoxia on maternal food intake or the qulity of the maternal milk for lactation. In addition, chickens have a short incubation period (21 days), meaning embryonic studies can be conducted quickly. Maternal lives do not have to be taken in order to study the fetus and so for a fetal study the use of chickens could be described as more ethical, reducing the number of animal use, thereby complying with the $3 \mathrm{R}$ principle enshrined by the Home Office. The relatively large size of the chicken embryo at term ( $>25 \mathrm{~g}$ on day 19 , term is 21 days) compared to the rodent fetus ( $<4 \mathrm{~g}$ on day 20 , term is 21 days) means that the chicken embryo model has the added advantage of facilitating study of cardiovascular function in ovo or in isolated organs, for instance by using the Langendorff preparation or the myograph (8). Interestingly, and perhaps surprisingly, the chronology of cardiac development in the chicken is much more comparable to the human than is the rodent, in which cardiac development continues into the postnatal period (8). Rodents are also polytocous, and the physiology of multiple pregnancies can be quite different to singletons, with different adaptations in place to support more 
than one fetus (8). Combined, these advantages make the chicken embryo an animal model to isolate the effects of chronic hypoxia on cardiovascular development and programming of cardiovascular dysfunction.

The data provided by the chicken studies performed in this thesis adds considerably to the existing body of literature to demonstrate that chronic fetal hypoxia can programme a developmental origin of cardiovascular disease independent of any effects of undernutrition. This is important because of the similarities between the patterns of cardiovascular disease in hypoxic animals and the cardiovascular disease found in human offspring of complicated pregnancy. For example, IUGR human fetuses and neonates show decreased cardiac ejection force, diastolic dysfunction, decreased cardiomyocyte numbers, hypertrophic hearts and increased aortic stiffness (8-10). Some factors, including aortic stiffness and endothelial dysfunction have also been reported to be more prevalent in adults who were growth restricted as fetuses (11). Whilst IUGR is not necessarily caused by chronic fetal hypoxia alone, the fact that animal models which isolate the effects of chronic hypoxia produce similar phenotypes confirms that chronic fetal hypoxia is likely to be an important factor in the correlations between complicated human pregnancy, IUGR and an increased risk of cardiovascular disease later in life.

\section{Target groups}

This sound knowledge base generated in this $\mathrm{PhD}$ study has the potential to significantly facilitate the national and international development of the field, providing a significant academic advance within the field and between related disciplines. The $\mathrm{PhD}$ candidate and supervisors attend national and international scientific meetings routinely and are deeply involved in undergraduate and graduate teaching. Therefore, we will refer to this $\mathrm{PhD}$ project at least at three levels: 1) at international and national scientific meetings; 2) in laboratory meetings to postgraduate and graduate students and technicians, and 3) in lectures to undergraduate students, especially those in their final year who may be contemplating potential $\mathrm{PhD}$ research projects. It is important that investment is made to train new researchers, particularly in the vulnerable skills of fetal surgery and studying fetal cardiovascular function. To deliver maximum benefit in development of skills, capacity and capability, this $\mathrm{PhD}$ work will also serve as the basis to: 1) Provide cross-disciplinary training for emerging scientists in all 
components of the thesis, spanning in vivo cardiovascular experimentation and stereological and histological analyses of the cardiovascular system; 2) Ensure that other scientists worldwide are exposed to the expertise available through international communications at scientific meetings; 3) Ensure that other scientists at the University of Cambridge, University of Maatricht and The Bolivian Institute for High Altitude Biology (IBBA) are exposed to the expertise available through maintained international collaboration. Thereore, this $\mathrm{PhD}$ also serves in outreach programmes.

The sound knowledge base generated in this $\mathrm{PhD}$ has also been published in high impact journals as original research articles. Combinations of original articles have been further disseminated in Topical Reviews and letters to the Editor of high impact journals. To further achieve excellence with impact, the University Communications Office at Cambridge has been alerted of the potential influence for human health of the scientific findings. Therefore, the work has had an immediate impact on clinicians, basic scientists, healthcare professionals, expectant mothers and their families in terms of providing them with information on the effects on the health of the offspring of high altitude pregnancy or reductions in fetal oxygenation in sea level complicated pregnancy. In addition, the data will benefit the design of therapeutic strategies to protect pregnancy complicated by chronic fetal hypoxia and/or improve fetal growth and development in adverse pregnancy with drugs that limit the adverse effects of fetal hypoxia, such as with spcific antioxidants. The proposed research is therefore likely to be of significant interest and benefit not only to researchers carrying out similar or related research in the field, but also to national and international researchers in other disciplines, such as experts in the metabolic syndrome, diabetes and gestational diabetes, scientists in biochemistry, pharmacology and nanotechnology, as well as cross-disciplinary teams in the pharmaceutical industry. Therefore, the data generated directly and indirectly has the potential to reduce the burden of cardiovascular disease throughout the life course, thereby having a major clinical, economic and societal impact on health.

\section{Conclusion}

It is now established that adverse conditions during pregnancy can trigger a fetal origin of cardiovascular dysfunction and/or increase the risk of heart disease in later life. Sub-optimal environmental conditions during early life that may promote 
the development of cardiovascular dysfunction in the offspring include alterations in fetal oxygenation and nutrition as well as fetal exposure to stress hormones, such as glucocorticoids. There has been growing interest in identifying the partial contributions of each of these stressors to programming of cardiovascular dysfunction. However, in humans and in many animal models this is difficult, as the challenges cannot be disentangled. By using the chicken embryo as an animal model and intertwining this with high altitude incubation, this $\mathrm{PhD}$ has been able to circumvent a number of problems. The work has isolated an important direct contribution of chronic fetal hypoxia in regulating fetal growth, cardiovascular and endocrine development as well as in the programming of systemic and pulmonray vascular disease in the adult offspring.

\section{References}

1. https://www.bhf.org.uk/research/heart-statistics

2. Nabel EG. Cardiovascular disease. N Engl J Med 2003; 349:60-72.

3. Gluckman PD, Hanson MA, Cooper C, Thornburg KL. Effect of in utero and early-life conditions on adult health and disease. N Engl J Med. 2008; 359(1):61-73.

4. Giussani DA, Niu Y, Herrera EA, Richter HG, Camm EJ, Thakor AS, Kane AD, Hansell JA, Brain KL, Skeffington KL, Itani N, Wooding FB, Cross CM, Allison BJ. Heart disease link to fetal hypoxia and oxidative stress. Adv Exp Med Biol. 2014; 814:77-87. Review.

5. Giussani DA, Davidge ST. Developmental programming of cardiovascular disease by prenatal hypoxia. J Dev Orig Health Dis 2013; 4(5), 328-37. Review.

6. Giussani DA. The fetal brain sparing response to hypoxia: physiological mechanisms. J Physiol. 2016; 594(5):1215-30. Review.

7. Giussani DA, Camm EJ, Niu Y, Richter HG, Blanco CE, Gottschalk R, Blake EZ, Horder KA, Thakor AS, Hansell JA, Kane AD, Wooding FB, Cross CM, Herrera EA. Developmental programming of cardiovascular dysfunction by prenatal hypoxia and oxidative stress. PLoS One 2012; 7(2):e31017.

8. Itani N, Salinas CE, Villena M, Skeffington KL, Beck C, Villamor E, Blanco $\mathrm{CE}$, Giussani DA. The highs and lows of programmed cardiovascular disease by developmental hypoxia: studies in the chicken embryo. J Physiol. 2017 Oct 5. doi: 10.1113/JP274111. 
9. Rizzo G, Capponi A, Rinaldo D, Arduini D \& Romanini C. Ventricular ejection force in growth-retarded fetuses. Ultrasound Obstet Gynecol 1995; 5, 247 255.

10. Veille JC, Hanson R, Sivakoff M, Hoen H \& Ben-Ami M. Fetal cardiac size in normal, intrauterine growth retarded, and diabetic pregnancies. Am J Perinatol 1993; 10, 275-279.

11. Leeson CP, Kattenhorn M, Morley R, Lucas A \& Deanfield JE. Impact of low birth weight and cardiovascular risk factors on endothelial function in early adult life. Circulation 2001; 103, 1264-1268. 


$$
\overline{4}
$$





\section{Acknowledgements}

To God all mighty, for giving me the opportunity to obtain this personal achievement and giving me the health, wisdom, and understanding to achieve this goal.

To my dear mother Miriam Salmón Salinas, for educating me and guiding in realizing my goals in life and giving me support at all times. Thank you for being there for me and God bless you always.

To my father Carlos Salinas Mercado, who even if he is not physically here, blesses and protects me from Heaven.

To my wife and daughters, thank you for being my motivation and inspiration. You have helped me on this journey, and this achievement is more yours than mine.

To the Universities of Maastricht, Cambridge and the Mayor University of San Andres for giving me this possibility. I feel proud to have taken one more step in my academic formation.

To my trainers and academic advisers: Mercedes Villena, Carlos Blanco, Eduardo Villamor and in a very special way to Prof. Dino Giussani. They are people of great wisdom who have worked to help me to arrive at the place where I find myself. Thank you for your teachings, support, unconditional attention, and thank you for your great friendship.

To my brothers and sisters, uncles and aunts, cousins and nephews, and all my family that in any way or another celebrate this success, thank you for trusting in me and consider me an example of overcoming.

God bless all those people who have contributed to my person, as they are benefactors of unimaginable importance in my human condition. 


$$
-
$$



Carlos Eduardo Salinas Salmón achieved his Medical Doctor degree at the Universidad Mayor de San Andrés, Bolivia in 1992. He finished his specialization in Cardiology in 1997 at the same University. Since that time, he has been a researcher at the Bolivian Institute for High-Altitude Biology in La Paz, Bolivia. Since 1997 Carlos has been Assistant Professor at the department of Cardiology and in 2010 he completed a master in Health Education at the Universidad Mayor de San Andrés. Presently, he is the director of the Bolivian Institute for HighAltitude Biology in La Paz, Bolivia.

\section{List of publications}

Itani N, Salinas CE, Villena M, Skeffington KL, Beck C, Villamor E, Blanco CE, Giussani DA. The highs and lows of programmed cardiovascular disease by developmental hypoxia: studies in the chicken embryo. J Physiol. 2017 Oct 5. doi:10.1113/JP274111. [Epub ahead of print]

Rimoldi SF, Rexhaj E, Villena M, Salinas-Salmon C, Allemann Y, Scherrer U, Sartori C. Novel Insights into Cardiovascular Regulation in Patients with Chronic Mountain Sickness. Adv Exp Med Biol. 2016;903:83-100.

Brenner R, Pratali L, Rimoldi SF, Murillo Jauregui CX, Soria R, Rexhaj E, Salinas Salmón C, Villena M, Romero C, Sartori C, Allemann Y, Scherrer U. Exaggerated pulmonary hypertension and right ventricular dysfunction in high-altitude dwellers with patent foramen ovale. Chest. 2015 Apr;147(4):1072-1079.

Julian CG, Pedersen BS, Salinas-Salmon C, Yang IV, Gonzales M, Vargas E, Moore LG,Schwartz DA. Unique DNA Methylation Patterns in Offspring of Hypertensive Pregnancy. Clin Transl Sci. 2015 Dec;8(6):740-5.

Julian CG, Gonzales M, Rodriguez A, Bellido D, Salinas-Salmon C, Ladenburger A, Reardon L, Vargas E, Moore LG. Perinatal hypoxia increases susceptibility to highaltitude polycythemia and attendant pulmonary vascular dysfunction. Am J Physiol Heart Circ Physiol. 2015 Aug 15;309(4):H565-73. 
Salinas CE, Blanco CE, Villena M, Giussani DA. High-altitude hypoxia and echocardiographic indices of pulmonary hypertension in male and female chickens at adulthood. Circ J. 2014;78(6):1459-64.

Herrera EA, Salinas CE, Blanco CE, Villena M, Giussani DA. High altitude hypoxia and blood pressure dysregulation in adult chickens. J Dev Orig Health Dis. 2013 Feb;4(1):69-76.

Bailey DM, Rimoldi SF, Rexhaj E, Pratali L, Salinas Salmòn C, Villena M, McEneny J, Young IS, Nicod P, Allemann Y, Scherrer U, Sartori C. Oxidative-nitrosative stress and systemic vascular function in highlanders with and without exaggerated hypoxemia. Chest. 2013 Feb 1;143(2):444-451.

Pratali L, Allemann Y, Rimoldi SF, Faita F, Hutter D, Rexhaj E, Brenner R, Bailey DM, Sartori C, Salinas-Salmon C, Villena M, Scherrer U, Picano E, Sicari R. RV contractility and exercise-induced pulmonary hypertension in chronic mountain sickness: a stress echocardiographic and tissue Doppler imaging study. JACC Cardiovasc Imaging. 2013 Dec;6(12):1287-97.

Salinas CE, Villena M, Blanco CE, Giussani DA. Adrenocortical suppression inhighland chick embryos is restored during incubation at sea level. High Alt Med Biol. 2011 Spring;12(1):79-87.

Pratali L, Rimoldi SF, Rexhaj E, Hutter D, Faita F, Salinas-Salmon C, Villena M, Sicari R, Picano E, Allemann Y, Scherrer U, Sartori C. Exercise induces rapid interstitial lung water accumulation in patients with chronic mountain sickness. Chest. 2012 Apr;141(4):953-8.

Rimoldi SF, Rexhaj E, Pratali L, Bailey DM, Hutter D, Faita F, Salinas-Salmon C, Villena M, Nicod P, Allemann Y, Scherrer U, Sartori C. Systemic vascular dysfunction in patients with chronic mountain sickness. Chest. 2012 Jan;141(1):139-46.

Jayet PY, Rimoldi SF, Stuber T, Salinas-Salmon C, Hutter D, Rexhaj E, Thalmann S, Schwab M, Turini P, Sartori-Cucchia C, Nicod P, Villena M, Allemann Y, Scherrer 
U, Sartori C. Pulmonary and systemic vascular dysfunction in young offspring of mothers with preeclampsia. Circulation. 2010 Aug 3;122(5):488-94.

Stuber T, Sartori C, Schwab M, Jayet PY, Rimoldi SF, Garcin S, Thalmann S, Spielvogel H, Salinas-Salmon C, Villena M, Scherrer U, Allemann Y. Exaggerated pulmonary hypertension during mild exercise in chronic mountain sickness. Chest. 2010 Feb;137(2):388-92.

Salinas CE, Blanco CE, Villena M, Camm EJ, Tuckett JD, Weerakkody RA, Kane AD, Shelley AM, Wooding FB, Quy M, Giussani DA. Cardiac and vascular disease prior to hatching in chick embryos incubated at high altitude. J Dev Orig Health Dis. 2010 Feb;1(1):60-6.

Stuber T, Sartori C, Salinas-Salmon C, Hutter D, Thalmann S, Turini P, Jayet PY, Schwab M, Sartori-Cucchia C, Villena M, Scherrer U, Allemann Y. Respiratory nitric oxide and pulmonary artery pressure in children of aymara and European ancestry at high altitude. Chest. 2008 Nov;134(5):996-1000.

Giussani DA, Salinas CE, Villena M, Blanco CE. The role of oxygen in prenatal growth: studies in the chick embryo. J Physiol. 2007 Dec 15;585(Pt 3):911-7. Epub 2007 Oct 25.

Scherrer U, Turini P, Thalmann S, Hutter D, Salinas-Salmon C, Stuber T, Shaw S, Jayet PY, Sartori-Cucchial C, Villena M, Allemann Y, Sartori C. Pulmonary hypertension in high-altitude dwellers: novel mechanisms, unsuspected predisposing factors. Adv Exp Med Biol. 2006;588:277-91. 

